\title{
Optimization of transcranial direct current stimulation (tDCS) to modulate lower limb motor network in healthy humans
}

\author{
Dissertation \\ zur Erlangung des humanwissenschaftlichen Doktorgrades \\ in der Medizin \\ der Georg-August-Universität Göttingen
}

vorgelegt von

Soares Foerster, Águida

Aus

Brasilien

Göttingen, 2018 


\section{Doctoral Thesis Committee}

\section{Prof. Dr. Nitsche, Michael (Supervisor)}

Dept. Psychology and Neurosciences

Leibniz Research Center for Working Environment and Human Factors

Ardeystr 67

44139 Dortmund

Prof. Dr. Niklas, Andree (Thesis reviewer)

Georg August University Göttingen

Institut für Sportmedizin

Sprangerweg 2

37075 Göttingen

Prof. Dr. Blumentritt, Siegmar (Thesis reviewer)

PFH Private Hochschule Goettingen

ZHT Zentrum für Healthcare Technology

Robert-Koch-Strasse 40

37075 Göttingen 


\section{Statement of Originality}

I hereby declare that this thesis "Optimization of transcranial direct current stimulation (tDCS) to modulate lower limb motor network function in healthy humans" was independently written and with no other sources and aids than quoted in the text, references and acknowledgements.

Goettingen,

12.06.2018

Soares Foerster, Águida 


\section{Table of Contents}

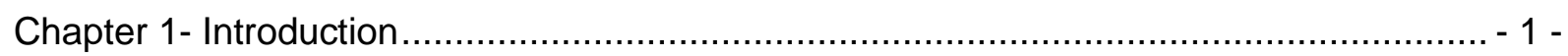

1.1.Lower limb motor control and corporal balance ................................................... 3 -

1.1.1. Lower limb motor control................................................................. 4 -

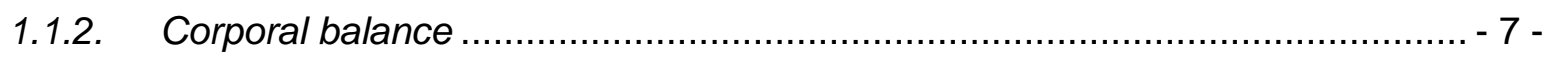

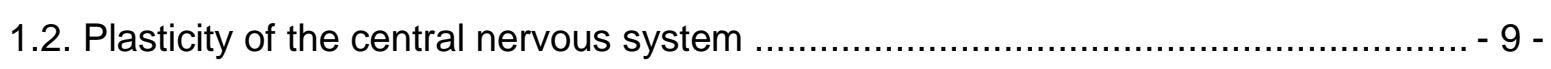

1.2.1. Neuroplasticity of the human primary motor cortex .................................. 12 -

1.3. Non-invasive brain stimulation in humans ........................................................ - 14 -

1.3.1. Transcranial magnetic stimulation (TMS) ................................................... 14 -

1.3.1.1. Motor evoked potential (MEP) ........................................................ - 15 -

1.3.2. Transcranial direct current stimulation (tDCS)....................................... - 16 -

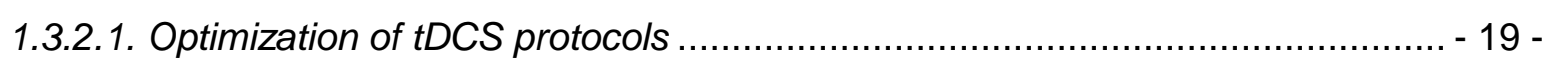

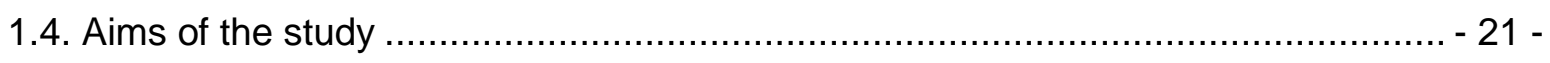

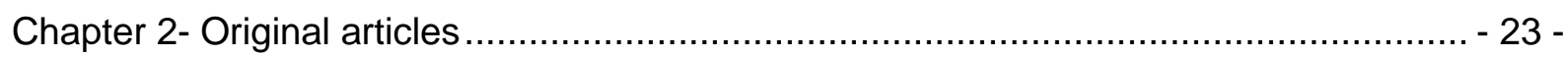

2.1. Effects of cathode location and the size of anode on anodal transcranial direct current stimulation over the leg motor area in healthy humans ........................................ 23 -

2.2. Effects of anodal transcranial direct current stimulation over lower limb primary motor cortex on motor learning in healthy individuals ................................................ 37 -

2.3. Cerebellar Transcranial Direct Current Stimulation (ctDCS) Impairs Balance Control in

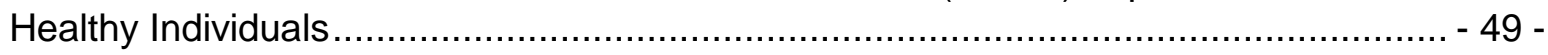

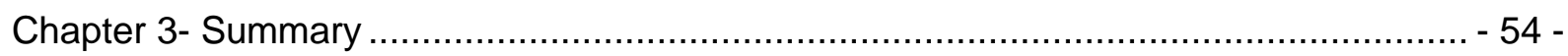

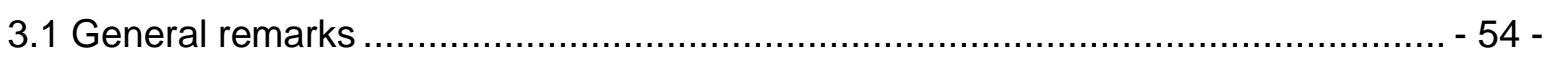

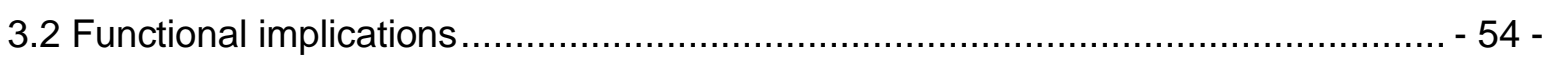

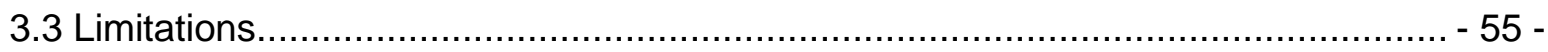

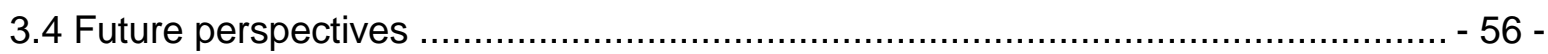

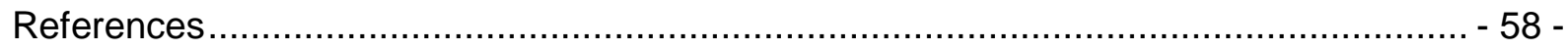

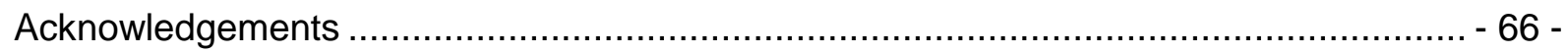




\section{Chapter 1- Introduction}

Human control of locomotion is a fascinating area of ongoing research, where physiologists, neuroscientists and engineers are working to increase the understanding of the complex pattern of neural commands involved in the control of lower limb movements. Parts of this central nervous system motor network are the primary motor cortex, premotor areas, parietal cortex, basal ganglia, thalamus, and cerebellum. These areas dynamically interact during locomotor movements, such as reaching, walking, and postural control. Neurons in the motor cortex command the changes in muscle activity required for lower limb movements, and maintainance of postural equilibrium in daily life. Simultaneously, neurons in the brainstem reticular formation ensure that these modifications are superimposed on an appropriate base of postural support (1).

Neuronal recordings and activation patterns revealed with neuroimaging methods have shown considerable plasticity of lower limb motor cortex representations and cell properties following pathological or traumatic changes and in relation to everyday experience, including motor skill (re-)learning (2). The process of motor (re-)learning for neurological patients depends on neuroplasticity, which is defined as the capacity of the brain to develop new neuronal/synaptic interconnections and thereby to develop and adapt new functions and roles or to reorganize to compensate for changes (3). Non-invasive brain stimulation (NIBS) has been shown to be able to induce plasticity in the human brain (4). Transcranial application of weak direct currents (tDCS) is one of the respective NIBS tools. Its primary mechanism is a stimulation polarity-dependent alteration of neuronal resting membrane potentials. Sufficiently long stimulation results in neuroplastic alterations 
of cortical excitability, and activity, which depend on the glutamatergic system, and share some features with learning-related plasticity (5).

Therefore, in recent years, the potential to combine tDCS with rehabilitation to improve motor recovery of neurological patients by modulating synaptic efficacy with tDCS emerged (6). Respective recovery processes are intrinsically linked to shifts in cortical excitability, which may share mechanisms with tDCS-induced neuromodulation $(7,8)$. In principal accordance, studies combining tDCS with primarily upper limb motor task performance in healthy individuals (9-11) and in neurological patients (12-14) improved performance. Based on these studies, it can be postulated that NIBS may improve also leg functions following neurological impairment (15). In fact, tDCS transiently elevated leg pinch-force of the nondominant leg of healthy subjects during and up to $30 \mathrm{~min}$ after its application (16). Furthermore, Madhavan and colleagues have shown that tDCS enhances motor control of the hemiparetic ankle in stroke patients (17). However, not much is known so far about tDCS protocols optimally suited to improve motor (re-) learning of lower limb functions (18).

In this project we were interested to explore the impact of tDCS over lower limb motor cortex representations on motor learning and cortex plasticity, and the influence of different stimulation parameters on motor cortex excitability in healthy individuals. Furthermore, we investigated the effect of cerebellar tDCS on corporal balance control. The first chapter introduces basic mechanisms relevant for understanding the studies included in the thesis. The second chapter consists of the publications presenting the research results. The concluding chapter summarizes the main results of the studies and offers an outlook to future research in the field. 


\subsection{Lower limb motor control and corporal balance}

Sequentially coordinated periodic extension and flexion movements of the hips, knees, and ankles are common to a number of human locomotor movements, such as ground level walking, running or stair climbing. The required sensorimotor control enabling these periodic movements is achieved by the interaction of proprioceptive feedback, the central pattern generator (CPG) at the spinal level, and higher-level control signals from cortical and subcortical supraspinal centers (Figure 1), i.e. premotor and motor cortex, cerebellum and brainstem (Duysens and Van De Crommert, 1998; Dietz, 2003; La Fougere et al., 2010). The latter regulates both the CPG and reflex mechanisms (Dietz, 2002). Recent findings from neuro-imaging studies indicate that the supraspinal areas might be involved in the control of gait to a higher extent than previously assumed (Miyai et al., 2001; Gwin et al., 2011). Also at the supraspinal level, information from vestibular and visual systems are incorporated, which are crucial for the maintenance of balance, orientation, and control of precise movement (Dietz, 2002).

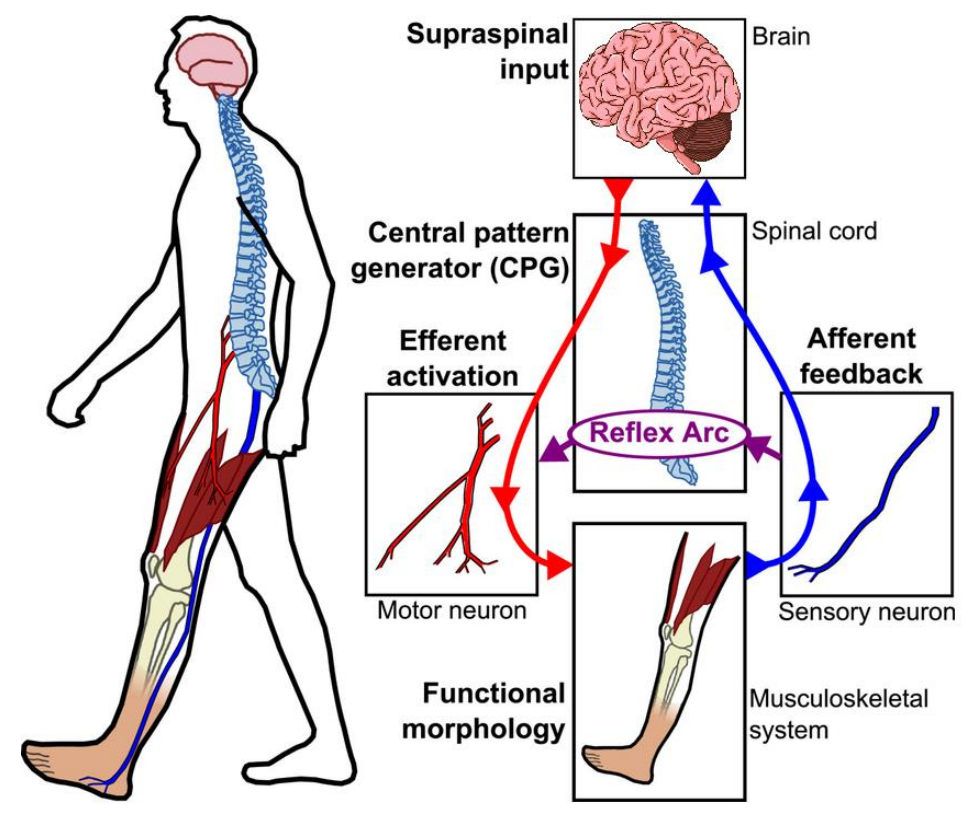

Figure 1. Nominal sensorimotor control loop for human locomotion [Adapted from Tucker et al., 2015 (19)]. 
Locomotor patterns are also modulated by afferent feedback arising from muscle spindles, Golgi tendon organs, mechanoreceptors lining the joint capsules, tactile mechanoreceptors and free nerve endings of the skin that sense stretch, pressure, heat, or pain (19). The modulation via reflex pathways is twofold: taking place under normal conditions, principally to increase the efficiency of gait, and during unexpected perturbations, to stabilize posture $(20,21)$.

Efferent nerve fibers, i.e. motor neurons, transmit the resulting motor commands to individual muscles, which are recruited to contract and thus to generate force on one or more joints of the skeletal system. Coordination of these forces through synergistic muscle activation and inter-joint coupling takes place during locomotor execution (22). Afferent nerve fibers, i.e. sensory neurons, transmit information from the musculoskeletal system to the central nervous system, thus closing the feedback loop for the nominal control of human locomotion (19).

\subsubsection{Lower limb motor control}

In order to execute a voluntary goal-directed motor task, the cerebral cortex communicates with the involved muscles via the corticospinal tract. The corticospinal neurons originate in the primary motor cortex (M1), project with their axons through the midbrain and pons, and decussate in the medulla to the opposite side of the spinal cord. The majority of these neurons terminates in the dorsolateral ventral horn of the spinal cord and communicates with interneurons or motoneurones (23). The respective motoneurones innervate multiple muscle fibers via neuromuscular junctions that convert the descending neural input into force output of a motor unit. Thus a motor unit is defined as all muscle fibres innervated by one motoneuron (24).

Fine control of voluntary movements employs the use of specific neural networks that are responsible for executing motor programs. Information from 
multiple areas of the cortex can influence motor output. For example, the primary motor cortex (M1), which produces and controls voluntary movements, receives information from the cerebellum (which coordinates movement), while the supplementary motor area (responsible for postural stabilization, sequencing of events) will receive input from the basal ganglia (which regulate inhibitory output to regulate movement) (23). Further, input from the prefrontal cortex, which receives and synthesizes input from the major sensory systems, basal ganglia and limbic system, provides information to the motor cortex via the premotor cortex to assist with planning, decision-making, and executive function tasks $(23,25)$. Executive functions (which include volition, planning, purposeful action, and action monitoring), anxiety, and stress are modulated in prefrontal cortical regions and the anterior cingulate cortex (26-29), and hereby affect motor activity (Figure 2).

CENTRAL NERVOUS SYSTEM PROCESS INVOLVED IN CONTROL OF POSTURE AND LOCOMOTION

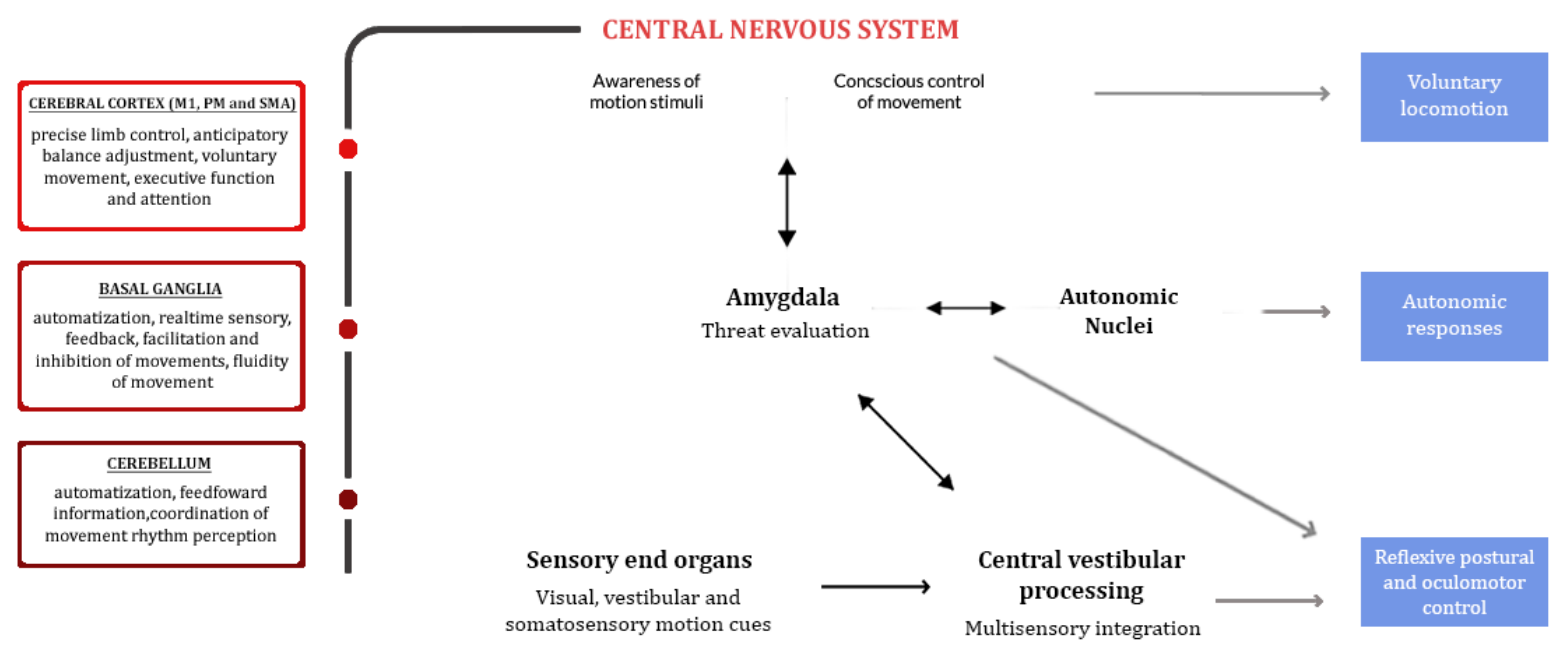

Figure 2. Brain network involved in lower limb motor function [Adapted from Staab et al., 2013 and Moon et al., $2016(30,31)]$.

While a basic locomotor rhythm is centrally generated by spinal circuits, descending pathways are critical for ensuring appropriate anticipatory modifications of gait to accommodate for uneven terrain (1). Studies have shown that the activity of 
a majority of cortical neurons, including those identified as projecting at least as far as to the more caudal regions of the pyramidal tract is modified during tasks that require skillful changes in gait. This includes e.g. fine-tuning of placing the foot accurately on the rungs of a horizontal ladder $(32,33)$, stepping over barriers on the ground (34), or attachment to a moving treadmill belt (35).

Movement of the lower limbs has been shown to correlate with changes in BOLD signal intensity (cortical activation) in M1 and the somatosensory cortex (36, 37). Active and passive ankle dorsiflexion and plantarflexion tasks activated similar cortical regions $(38,39)$, and graded dorsiflexion movements of the right ankle have produced graded BOLD signal changes in motor areas (40). This is indicative for the critical involvement of and interaction between these areas for respective movements. In order to better understand the cortical activation mechanisms during leg movements, and to identify the cortical network associated with control of the lower limb motor functions, invasive electrical stimulation and non-invasive brain stimulation (NIBS) have been used. Experiments in which the motor cortex, or pyramidal tract, have been stimulated during locomotion (41-43) suggest that the effects of a corticospinal volley are mediated by interneuronal pathways that are influenced by, or part of, the spinal CPG for locomotion (44). NIBS studies have shown that anodal transcranial current stimulation (tDCS), a type of NIBS, applied over the leg motor cortex, can influence corticomotor excitability of different structures that are considered to play a role in the control of walking $(15,45,46)$. Accordingly, tDCS leads to an increase in maximal voluntary pinch force, generated by the toes (16). Additionally, tDCS has been suggested to enhance activity of subcortical structures (47), as it accelerated automatic postural responses which arise from subcortical structures (48). 


\subsubsection{Corporal balance}

The ability to stand, and to walk depends on a complex interaction of physiological mechanisms involved in the neuronal control of corporal balance. Corporal balance can be defined as the ability to maintain a stable body position based on weight support, whether stationary or dynamic (49). The maintenance of balance is essential for the majority of motor activities in daily life. This includes rather automated processes such as the maintenance of an upright posture as well as more complex movements during sports or when balance is disrupted unpredictably. It is a motor skill mediated mainly by the extrapyramidal tract, which is discernable from the pyramidal tract (corticobulbar and corticospinal tracts) which pass through the pyramids of the medulla (50). The extrapyramidal tract is found in the reticular formation in the medulla and pons. Its target neurons are found in the spinal cord and are responsible for movement, walking and reflexes. This tract is influenced by pathways from the basal ganglia, sensory cortex, vestibular nuclei and also the cerebellum (51). Therefore, corporal balance control is considered a complex motor function, since it is dependent on the integration of a large central nervous system network $(52,53)$.

The cerebral cortex is involved in the central control of postural balance via two main loops, one including the cerebellum and one including the basal ganglia (Figure 3). Studies suggest that the cerebellar-cortical loop is responsible for adapting corporal balance based on prior experience, whereas the basal ganglia are responsible for pre-selecting and optimizing postural responses based on current context (54). 


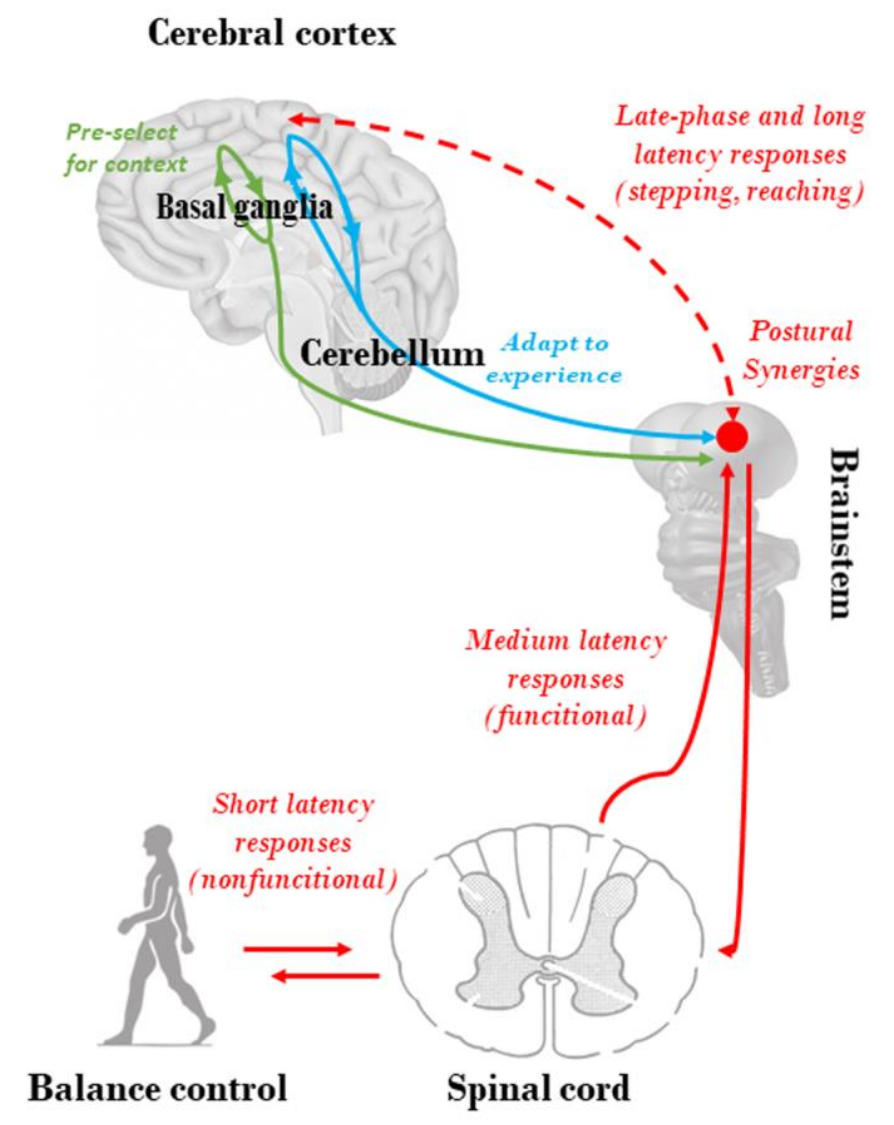

Figure 3. Neural pathways involved in central control of postural balance [Adapted from Jacobs and Horak, 2007(54)].

Through the processing of information from the spinal cord, brainstem, and cerebral cortex, the cerebellum is an important structure involved in static and dynamic balance control (55). The cerebellum is involved in adapting response magnitude and in tuning the coordination of postural responses based on practice and knowledge of results, similar to its contribution to adaptation and coordination of other movements (56). The cerebral cortex likely influences postural responses directly via corticospinal loops and indirectly via communication with the brainstem centers that harbor the synergies for postural responses, thereby providing both speed and flexibility for pre-selecting environmentally appropriate responses to a loss of balance (54). 


\subsection{Plasticity of the central nervous system}

One fundamental function of the central nervous system is to control voluntary movements. Recent evidence suggests that this role emerges from distributed networks rather than discrete representations and that in adult mammals these networks are undergo modifications that are moderated by plasticity mechanisms (57). Neuroplasticity can be broadly defined as the ability of the nervous system to respond to intrinsic and extrinsic stimuli by reorganizing its structure, function and connections; it can be described at many levels, from molecular to cellular to systems to behaviour; and occurs during development, in response to environmental demands, in response to disease, or as a consequence of therapy. Plasticity can be viewed as adaptive when associated with a gain of function (58), or as maladaptive when associated with negative consequences such as loss of function or increased injury, as illustrated by animal models and human studies (59). Also, adaptive plasticity should be distinguished from compensatory behaviours, which subsume the appearance of new motor patterns resulting from the adaptation of remaining motor elements or substitution, meaning that functions are taken over, replaced, or substituted by different effectors or body segments (60).

Functional neuronal plasticity is based on synaptic plasticity, which is the ability of the synapses to strengthen or weaken over time, in response to increases or decreases in their activity (61). Plasticity at synapses can be regulated at the presynaptic site by changing the release of neurotransmitters or postsynaptically by changing the number, types, or properties of neurotransmitter receptors (62). While most research attention is focused on the functional aspects of synaptic plasticity and their key contribution to learning and memory mechanisms, work in the last decade has also clearly demonstrated the importance of associated structural 
rearrangements. These consist of different types of morphological changes (enlargement, growth, pruning, stabilization), affecting different cellular compartments (spines, terminals, astrocytic processes), and take place on different time scales (minutes to days), making them sometimes difficult to relate to functional changes (63).

Since memories are postulated to be represented by vastly interconnected networks of synapses in the brain, synaptic plasticity is one of the important neurochemical foundations of learning and memory (61). Glutamatergic systems play a crucial role for synaptic plasticity relevant for learning and memory formation (64). Glutamate is the major excitatory neurotransmitter in the nervous system. Glutamate pathways are linked to many other neurotransmitter pathways, and glutamate receptors are found throughout the brain and spinal cord in neurons and glia (Altevogt et al., 2011). Studies using in vitro synaptic plasticity models have identified the regulated trafficking of postsynaptic alpha-amino-3-hydroxy-5-methyl-4isoxazolepropionate (AMPA) type glutamate receptors as a prevalent mechanism underlying activity-induced changes in synaptic transmission $(65,66)$. Excitatory synapses contain AMPA-type receptors to transmit signals and calcium-permeable $\mathrm{N}$-methyl-D-asparate (NMDA) type receptors to trigger long-term changes in synaptic transmission: long term potentiation (LTP) and long term depression (LTD). While different mechanisms can regulate the onset or magnitude of LTP and LTD, in many cases, there appears to be one common mechanism controlling the postsynaptic expression: the addition and removal, respectively, of synaptic AMPA receptors (67, $68)$.

At the cellular level, LTP and LTD are the most widely studied neuroplastic mechanisms considered to be fundamental for learning and memory formation (69, 
70). It is well known that increased postsynaptic intracellular calcium concentration is an important signal for the induction of LTP and LTD (71-73). High enhancement of intracellular calcium induces LTP, whereas low enhancement results in LTD (74). The mechanisms of synaptic alteration are in accordance to the rules of Hebbian plasticity, characterized by longevity, input specificity and associativity. Learning and memory formation are based on modifications of synaptic strength among neurons (75).

LTP and LTD processes are most detailed studied at glutamatergic synapses, especially in the region of the hippocampus, but also in other cortical and subcortical areas (73). Plasticity of the glutamatergic system is accomplished primarily via calcium-permeable NMDA receptors (70). LTP is accomplished by activation of postsynaptic NMDA receptors and calcium-dependent protein kinases which results in the above-mentioned postsynaptic insertion of AMPA receptors (72). LTD is generated by moderate activation of NMDA receptors and another type of calciumdependent enzymes which leads to the internalization of postsynaptic AMPA receptors (72).

These cellular mechanisms are important for adaptive reorganization of cortical networks of the brain following physiological or pathological changes (76). After cortical injury, the structure and function of undamaged parts of the brain are remodeled during recovery, and shaped by the sensorimotor experiences of the individual in the weeks to months following injury. This reorganization suggests that rehabilitative interventions may work via modulation of neuroplastic mechanisms (77). 


\subsubsection{Neuroplasticity of the human primary motor cortex}

The human motor system is reorganizing itself more or less permanently on the basis of input. This capacity to reorganize plays a crucial role not only in learning, but also in recovery of motor functions after damage to the brain (78). Human motor behavior is not the result of a stereotyped and static series of detailed musclespecific central commands, but is characterized by an extreme flexibility. It has been shown that neural representations of the limbs are flexible and continuously updated by body movements (79). Repetition of movement leads to the strengthening of motor cortex representations, whereas inactivity or non-use results in the shrinkage of these representations. This shows that the adult human brain is not a rigid system, but continuously undergoes plastic changes caused by alterations of the sensory flow from peripheral receptors and nerve fibers (78).

Recent evidence has directly demonstrated that the primary motor cortex (M1) contains a substrate for and a mechanism to implement plasticity, thereby placing the intrinsic circuitry of M1 in a key position to account for brain network (re-)organization during a new skill training process, or after a neuronal injury (57). The interactions between cortico-thalamic-striatal and cortico-thalamic-cerebellar structures and the limbic system, and the specific associative-premotor and sensorimotor networks, are essential for M1 to successfully modulate synaptic efficacy, and promote neuroplasticy (80).

Motor cortical plasticity has been studied in patients who had a unilateral immobilization of the ankle joint without any peripheral nerve lesion. The motor cortex area of the inactivated tibial anterior muscle dimished compared to the unaffected leg without changes in spinal excitability or motor threshold (81). This demonstrates that M1 has the intrinsic circuitry required for reorganization, and the results further 
suggest that the details of $\mathrm{M} 1$ organization likely depend, probably moment to moment, on the precise balance of excitatory and inhibitory influences within the network of M1 connections. Representations increase or decrease depending on use, and to determine how fast such changes can occur, motor learning can be investigated (2).

M1 networks seem to be active during different time points of motor learning (82-84). Motor learning can modulate functional connectivity of the cortical motor network, and early skill learning has been shown to lead to enhanced inter- and intrahemispheric coupling (85). M1 seems to play a crucial part in fast motor learning (86, 87). Rodent studies have shown that motor learning can induce recruitment of neurons in the M1 and modulate synaptic efficacy through LTP and LTD $(69,88-90)$. These results are supported by human studies, which also suggest that LTP-like plasticity in the M1 is involved in motor learning (91-93).

In humans, transcranial stimulation with electrical and magnetic devices has a been used to study M1 map plasticity (94-96). Transcranial magnetic stimulation (TMS), although with significantly less spatial resolution than intracortical techniques, has been established as a powerful mapping tool for clinical and research applications (97). TMS is a noninvasive technique that utilizes short, rapidly changing magnetic field pulses to induce electrical currents in underlying cortical tissue (98). A simple example of a TMS-based connectivity measure involves delivering a single TMS pulse to the primary motor cortex, and then measuring the induced contralateral muscle contraction in the form of a motor evoked potential (MEP). For the TMS pulse to reach the muscle it must cross synapses in the anterior horn of the spinal cord and at the neuromuscular junction (99). Non-invasive brain stimulation tools are probed as well as treatment approaches, since specific protocols are able to induce 
neuroplasticity, and thus are able to enhance training-induced cognitive and motor learning (3).

\subsection{Non-invasive brain stimulation in humans}

In the past three decades, our understanding of brain-behavior-relationships has been significantly improved by research using non-invasive brain stimulation (NIBS) techniques. These methods, such as TMS, repetitive transcranial magnetic stimulation (rTMS), transcranial direct current stimulation (tDCS), and paired associative stimulation (PAS), allow the non-invasive and safe modulation of neural processes in the healthy and pathologically altered brain, enabling researchers to directly study neural plasticiy and its association with behavioral alterations. Here, we introduce TMS and tDCS, which are related to our studies.

\subsubsection{Transcranial magnetic stimulation (TMS)}

Transcranial magnetic stimulation is a neurostimulation and neuromodulation technique, based on the principle of electromagnetic induction of an electric field in the brain. It was introduced by Anthony Barker in 1985 (100). The induced electrical field is of sufficient magnitude and density to depolarize neurons, leading to induction of cortical activity, in physiological and pathological conditions (101). TMS is thought to not activate corticospinal neurons directly; rather it activates them indirectly through synaptic inputs from intracortical neurons. This assumption is based on the observation that motor cortex TMS produces a corticospinal volley with indirect waves rather than with the early direct wave (102).

The design of TMS consists of a main stimulator and a stimulating coil, and it can be applied with one stimulus at a time, single-pulse TMS, in pairs of stimuli separated by a variable interval, paired-pulse TMS, or in trains, repetitive TMS. 
Single-pulse TMS can be used, for example, for mapping motor cortical outputs, studying central motor conduction time, and studying causal chronometry in brainbehavior relations. In paired pulse techniques, TMS can be delivered to a single cortical target using the same coil or to two different brain regions using two different coils. Paired pulse techniques can provide measures of intracortical facilitation and inhibition, as well as study cortico-cortical interactions (101). In our first two studies discussed below, single-pulse TMS was applied to the lower limb primary motor cortex (M1) for identification of the motor cortex representation of the tibialis anterior (TA) muscle, and to monitor its cortical excitability. This was done via recording of motor evoked potentials (MEP), which we introduce in more detail below.

\subsubsection{Motor evoked potential (MEP)}

The Motor evoked potential (MEP) is an electrical muscular response elicited after artificial stimulation of the corticospinal tract anywhere above the spinal motor neuron. Usually, it is induced by stimulation over the motor cortex via single-pulse TMS, and recorded via surface electromyography (EMG) (103). The amplitude of the MEP reflects not only the integrity of the corticospinal tract, but also the excitability of the motor cortex and nerve roots and the conduction along the peripheral motor pathway to the muscles (104).

To record MEP as a global measure of cortiço-spinal excitability, single-pulse TMS is applied to the primary motor cortex. To monitor excitability alterations of a target area due to an intervention, a baseline TMS intensity is defined which induces a moderately sized MEP amplitude of the target area (hot-spot), and then this intensity is kept constant throughout the experiment. Alterations of MEP amplitudes in this case index intervention-dependent excitability changes (105). Moreover, MEP amplitudes are altered after the application of modulators of inhibitory and excitatory 
transmission in neuronal networks. For instance, the MEP is depressed by modulators of GABAA receptors, but increased by dopamine agonists and various norepinephrine agonists (106).

\subsubsection{Transcranial direct current stimulation (tDCS)}

Over the past two decades, the interest in human brain stimulation through the use of galvanic current has been increased. The history of electrical brain stimulation goes back to the nineteenth century, when the first reports describing the application of an electric current to an isolated area of the exposed brain made cerebral stimulation a great neuroscientific novelty of that time $(107,108)$. In 1802, Giovanni Aldini concluded, after electrical stimulation of the meninges and cortical surface of the corpses of two recently decapitated prisoners, that the cortical surface was electrically excitable (109). In the mid-1960s, it was obeserved that the gradient of electrical potentials produced by low intensity continuous currents, which did not induce action potentials, was able to alter neural excitability and spontaneous activity $(110,111)$. In 1998, Priori and colleagues observed a suppression of cortical excitability in the human motor cortex after anodal stimulation, when preceeded by cathodal stimulation of the target area with weak direct currents (112). Transcranial direct current stimulation (tDCS), as currently applied, was introduced by Paulus and Nitsche in 2000. The authors demonstrated in a pioneering study the polaritydependent effect of tDCS on cortical excitability in the motor cortex of healthy subjects (113). Since then, tDCS has been widely used for therapeutic purposes, and the analysis of brain functions of healthy humans.

tDCS differs from the brain stimulation techniques applied in the early studies mentioned above, and from transcranial electrical stimulation and TMS, which induce neuronal firing by suprathreshold neuronal membrane depolarization (114). The 
principal mechanism of action of tDCS is a subthreshold modulation of neuronal membrane potentials, which alters cortical excitability and activity dependent on the current flow direction through the target neurons $(115,116)$ via immediate changes of neuronal firing by hyperpolarizing or depolarizing brain tissue $(117,118)$. It has been shown that tDCS also modifies the synaptic microenvironment, for instance, by modifying synaptic strength NMDA receptor-dependently and altering GABAergic activity $(117,119,120)$. tDCS interferes with brain excitability through modulation of intracortical and corticospinal neurons $(121,122)$. Sufficiently long stimulation moreover results in neuroplastic cortical excitability alterations, similar to LTP and LTD (113). The mechanistic aspects of the induction of LTP and LTD via tDCS is not fully understood, however, it is suggested that its effects occur by changes in the functional expression of proteins and depend mainly on the neuronal influx of calcium controlled by alterations of NMDA receptor activity $(74,123)$.

The aftereffect of tDCS is thought to modulate cortical excitability in a polarityspecific manner (5). Stimulation of M1 with an anode positioned over M1 hand area is usually reported to increase MEP size, while cathodal tDCS has the opposite effect (113). It is suggested that those excitability changes occur in the intracortical interneurons within the cortex. The aftereffects are dependent on modulation of both GABAergic and glutamatergic synapses. Anodal and cathodal tDCS reduce GABA, which might gate plasticity of glutamatergic synapses, which is controlled by tDCS. The respective stimulation-induced calcium alterations will then induce polaritydependent LTP- or LTD-like plasticity dependent on the mechanisms described above (118).

The neurophysiological effects outlast the stimulation period by up to $90 \mathrm{~min}$ $(113,124)$. The duration, strength and direction of the effects depend on the duration, 
polarity and intensity of tDCS. The expected effects of polarity on excitability (excitability enhancment after anodal stimulation, and decrease after cathodal tDCS) are observed after tDCS application of durations between 5 and 20 min using $1 \mathrm{~mA}$ (5). Further prolongation of duration or increasing intensity of stimulation can reverse the after-effects $(125,126)$.

tDCS has been used as a probe to modulate attention, memory, motor, and language functions in humans, based on respective excitability and neuroplasticity alterations. On the basis of human neuroimaging studies, it was proposed that application of noninvasive stimulation with parameters that enhance motor cortical excitability, and plasticity could secondarily facilitate motor learning via boosting respective task-associated cortical alterations (127). Motor skill learning and adaptation are associated with functional and structural changes of a distributed brain network that includes primary motor, somatosensory, premotor, supplementary motor and posterior parietal cortex, as well as the cerebellum and basal ganglia (128130). Thus, several candidate brain networks are accessible to tDCS for investigating neuromodulatory effects on different features of motor learning (131). The effects of tCDS on motor learning seem to be strongest when tDCS is co-applied with motor training $(132,133)$ and applied over multiple days (134-136).

Although most early tDCS studies have been performed in the motor cortex (i.e. M1), it should be noticed that tDCS does not only induce long-lasting alterations of motor-evoked potentials, but also affects somatosensory and visual-evoked potentials (114). It has been observed that tDCS can influence the human cerebellum $(137,138)$, and that transcutaneous DC stimulation modulates conduction along the spinal cord and the segmental reflex pathways $(139,140)$. 
tDCS does not induce activity in resting neuronal networks, but modulates spontaneous neuronal activity (141). Consequently, the amount and direction of stimulation effects critically depend on the previous physiological state of the target neural structures $(142,143)$. This brain-state dependency could possibly explain interindividual variability of tDCS effects previously reported $(144,145)$ and the fact that differences in experimental protocols such as stimulation intensity or use of different behavioral tasks result in different outcomes $(125,142)$.

\subsubsection{Optimization of tDCS protocols}

Current protocols of tDCS use relatively standardized stimulation parameters (electrode size of $25-35 \mathrm{~cm}^{2}$, current of 1-2 mA for up to 15-40 min, administered in multiple or single sections), which have been demonstrated to be safe $(5,118,146)$. Considering that differences in stimulation protocols could result in distinct brain current flow patterns across the brain, tDCS dose parameters can be adjusted, in an application-specific manner, to target or avoid specific brain regions (147). Variability in tDCS results has been obersed. Several reasons that may explain this variability in the tDCS results may include (i) use of different stimulation parameters (current density, duration and electrode geometry) and (ii) differences across individuals (146). In order to better understand this variable effect of tDCS the interest to develop optimized tDCS protocols has been growing.

The conventional strategy is to apply a continuous current via two rectangular electrodes, with one electrode placed over the target region and the other electrode placed remotely from the target region (148). The location of the electrodes is typically based on the International 10-20 EEG measurement system or electrophysiological markers, such as the motor hotspot defined by TMS (146). Because tDCS uses electrodes placed on the scalp to inject current, it is difficult to 
precisely control the current flow in the head and brain in order to elicit the desired current density in a target brain region of interest (ROI). In particular, current delivery to the $\mathrm{ROI}$ is limited due to shunting via the scalp, cerebrospinal fluid (CSF), gyral depth, distance between anode and cathode, and electrode connector positions (149). At a constant current intensity level, differences in electrode size, configuration, and placement result in different distribution of the current across the $\mathrm{ROI}$, and throughout the brain $(150,151)$. Therefore, not only the current intensity applied is critical to the tDCS results, but the shape, the size, the placement of the electrodes, and also the amount of contact medium (e.g. saline, gel or conductive cream) used has to be taken in account.

In order to target as precisely as possible the ROI and optimize tDCS protocols, modelling systems based on finite element head models have been created to investigate the induced current density distribution by analysis of electrical field orientation (152-154). Taking the dependency of tDCS effects from the relation of electrical field and neural spatial orientation into account, it is important to calculate the distribution of electric field strength and orientation via computational modelling. Considering that the components of the field perpendicular and parallel to the cortical surface are of special importance, since pyramidal cells are mostly aligned perpendicular to the surface, while many cortical interneurons and axonal projections of pyramidal cells tend to align tangentially $(155,156)$, an important element in modeling is to provide the electric field distribution and orientation relative to the grey matter (GM) and white matter (WM) surfaces. The use of modelling systems to investigate the impact of stimulation electrode shape, placement and size of the electrodes on electrical field distribution is thus an important tool to optimize tDCS effects on cortical excitability and behaviour. 


\subsection{Aims of the study}

The purpose of this work was to investigate the impact of tDCS applied over the lower limb motor network on cortical excitability, motor learning, and corporal balance control in healthy humans. According to the literature, tDCS effects are stimulation parameter-dependent. In our first study, we investigated the effect of electrode size, and placement on lower limb motor cortex excitability in healthy subjects, for optimization of tDCS effcts over the lower limb motor cortex representation by systematically exploring the impact of electrode size, and current flow direction based on computational modeling.

It was shown that administration of tDCS over M1 enhances motor performance, associated with respective physiological alterations, via its impact on cortical excitability, and plasticity. Most of these studies were however conducted for tDCS applied over the upper limb motor cortex area. Taking into account the importance of lower limb motor functions for daily life, for the second study, we explored the impact of tDCS on performance of a visuo-motor lower limb motor learning task in healthy humans. Based on the relevance of stimulation focality, which is particularly challenging for cortical areas remote from the brain surface as the leg motor cortex respesentation, we investigated the specificity of tDCS by finite element modeling regarding two different sizes of electrodes $\left(8 \mathrm{~cm}^{2}\right.$ vs. $\left.35 \mathrm{~cm}^{2}\right)$. As tDCS had interindividual heterogeneous effects on motor performance, and sensitivity to transcranial magnetic stimulation (TMS) has been revealed as a potential marker of responsivity to tDCS for the upper limb motor cortex (157), we furthermore aimed to explore the relevance of this parameter for the stimulation effects.

Beyond its impact on motor cortex plasticity, recent studies have shown that tDCS applied over the cerebellum (ctDCS) impacts also on motor functions in 
humans, thus for the third study, we explored the effects of cerebellar tDCS on corporal balance in healthy humans. The impact of tDCS on performance was explored via tests of static (right and left Athlete Single Leg tests) and dynamic balance (Limits of Stability test) performed using the Biodex Balance System before and immediately after cerebellar tDCS. The knowledge we aimed to gain via these studies might perspectively help to optimize the effects of tDCS on cortical plasticity and motor (re-) learning for clinical therapeutic interventions. 


\section{Chapter 2- Original articles}

This chapter contains three published articles. The first and the second study focused on optimized tDCS protocol effects over lower limb motor cortex excitability and motor learning in healthy humans. The third study investigated the effect of cerebellar tDCS over balance control in healthy individuals.

I. Foerster ÁS, Rezaee, Z, Paulus W, Nitsche MA, Dutta A. (2018). Effects of cathode location and the size of anode on anodal transcranial direct current stimulation over the leg motor area in healthy humans. Frontiers of Neuroscience (Published).

II. Foerster Á, Dutta A, Kuo MF; Paulus W, Nitsche MA. (2018). Effects of anodal transcranial direct current stimulation over lower limb primary motor cortex on motor learning in healthy individuals. European Journal of Neuroscience. 2018 Feb 14. doi: 10.1111/ejn.13866 (Published).

III. Foerster Á, Melo L, Mello M, Castro R, Shirahige L, Rocha S, Monte-Silva K. (2017). Cerebellar Transcranial Direct Current Stimulation (ctDCS) Impairs Balance Control in Healthy Individuals. Cerebellum 16(4):872-875 (Published).

\subsection{Effects of cathode location and the size of anode on anodal transcranial direct current stimulation over the leg motor area in healthy humans}

The efficacy of transcranial direct current stimulation to induce physiological effects depends on different stimulation aspects, such as current density, electrode size, electrode placement/configuration, stimulation duration, and combination with task performance or rehabilitation therapy. The conventional tDCS strategy is to apply a continuous current via two rectangular electrodes, with one electrode placed over the target region and the other electrode placed remotely from the target region $(113,148,158)$. Modelling systems based on finite element head models have been 
created to investigate the induced current density distribution (152-154). The location of the electrodes is typically based on the International 10-20 EEG measurement system or electrophysiological markers, such as the motor hotspot defined by transcranial magnetic stimulation (TMS). In this study, we investigated the effects of cathode location and the size of anode for anodal tDCS of the right leg area of the motor cortex, which is challenging due to its depth and orientation in the interhemispheric fissure. We first computationally investigated the effects of cathode location and the size of the anode to find the best montage for specificity of stimulation effects for the targeted leg motor area using finite element analysis (FEA). We then compared the best electrode montage found from FEA with the conventional montage (contralateral supraorbital cathode) via neurophysiological testing of both, the targeted as well as the contralateral leg motor area. The conventional anodal tDCS electrode montage for leg motor cortex stimulation with a large-anode $(5 \mathrm{~cm} \times 7 \mathrm{~cm}$, current strength $2 \mathrm{~mA})$ affected the contralateral side more strongly in both the FEA and the neurophysiological testing when compared to the other electrode montages. A small anode $(3.5 \mathrm{~cm} \times 1 \mathrm{~cm}$, current strength $0.2 \mathrm{~mA})$ with the same current density at the electrode surface and identical contralateral supraorbital cathode placement improved specificity. The best cathode location for the small anode in terms of specificity for anodal tDCS of the right-leg motor area was T7 (1010 EEG system). Our results show that a small-anode $(3.5 \mathrm{~cm} \times 1 \mathrm{~cm})$ with the same current density at the electrode surface as a large anode $(5 \mathrm{~cm} \times 7 \mathrm{~cm})$ resulted in similar cortical excitability alterations of the targeted leg motor cortex respesentation while the small anode with the cathode placed at T7 resulted in the best specificity. These results might help to optimize future studies targeting modulation of lower limb motor cortex representations via tDCS. 
OPEN ACCESS

Edited by: Mikhail Lebeder,

Duke University, United States

Reviewed by: Marco lacoboni

University of California, Los Angeles,

United States

Vassiliy Tsytsarev,

University of Maryland, College Park,

United States

Mayank A. Jog,

Keck School of Medicine of USC

United States

${ }^{*}$ Correspondence:

Águida S. Foerster

foerster.as@gmail.com;

foerster@ifado.de

Anirban Dutta

anirband@buffalo.edu

adutta@case.edu

Specialty section: This article was submitted to

Neural Technolog

a section of the journa

Frontiers in Neuroscience

Received: 27 October 2017

Accepted: 12 June 2018

Published: 04 July 2018

Citation:

Foerster ÁS, Rezaee Z, Paulus W,

Nitsche MA and Dutta A (2018)

Effects of Cathode Location

and the Size of Anode on Anoda

Transcranial Direct Current Stimulation

Over the Leg Motor Area in Healthy

Humans. Front. Neurosci. 12:443.

doi: 10.3389/fnins.2018.00443

\section{Effects of Cathode Location and the Size of Anode on Anodal Transcranial Direct Current Stimulation Over the Leg Motor Area in Healthy Humans}

Águida S. Foerster ${ }^{1,2 *}$, Zeynab Rezaee ${ }^{3}$, Walter Paulus ${ }^{1}$, Michael A. Nitsche ${ }^{2,4}$ and Anirban Dutta ${ }^{3 *}$

' Department of Clinical Neurophysiology, Universitätsmedizin Göttingen, Georg-August-Universität Göttingen, Göttingen Germany, ${ }^{2}$ IfADo - Leibniz Research Center for Working Environment and Human Factors, Dortmund, Germany,

${ }^{3}$ Department of Biomedical Engineering. University at Buffalo, The State University of New York (SUNY, Buffalo, NY,

United States, " Department of Neurology, BG University Hospital Bergmannsheil, Bochum, Germany

Objective: Non-invasive brain stimulation such as transcranial direct current stimulation (tDCS) involves passing low currents through the brain and is a promising tool for the modulation of cortical excitability. In this study, we investigated the effects of cathode location and the size of anode for anodal tDCS of the right-leg area of the motor cortex, which is challenging due to its depth and orientation in the inter-hemispheric fissure.

Methods: We first computationally investigated the effects of cathode location and the size of the anode to find the best montage for specificity of stimulation effects for the targeted leg motor area using finite element analysis (FEA). We then compared the best electrode montage found from FEA with the conventional montage (contralateral supraorbital cathode) via neurophysiological testing of both, the targeted as well as the contralateral leg motor area.

Results: The conventional anodal tDCS electrode montage for leg motor cortex stimulation using a large-anode $(5 \mathrm{~cm} \times 7 \mathrm{~cm}$, current strength $2 \mathrm{~mA})$ affected the contralateral side more strongly in both the FEA and the neurophysiological testing when compared to other electrode montages. A small-anode $(3.5 \mathrm{~cm} \times 1 \mathrm{~cm}$ at $0.2 \mathrm{~mA})$ with the same current density at the electrode surface and identical contralateral supraorbital cathode placement improved specificity. The best cathode location for the small-anode in terms of specificity for anodal tDCS of the right-leg motor area was T7 (10-10 EEG system).

Conclusion: A small-anode $(3.5 \mathrm{~cm} \times 1 \mathrm{~cm})$ with the same current density at the electrode surface as a large-anode $(5 \mathrm{~cm} \times 7 \mathrm{~cm})$ resulted in similar cortical excitability alterations of the targeted leg motor cortex respresentation. In relation to the other stimulation conditions, the small-anode montage with the cathode positioned at T7 resulted in the best specificity.

Keywords: lower limb motor cortex, stimulation parameters, motor cortex excitability, modeling, transcranial direct current stimulation (tDCS) 


\section{INTRODUCTION}

Clinical applications of non-invasive brain stimulation (NIBS) are currently an evolving area and increasingly used as an adjuvant treatment during motor rehabilitation (Flöel, 2014). Transcranial direct current stimulation (tDCS) is a NIBS modality that involves application of low intensity direct currents using two or more electrodes for a certain duration, which can alter corticospinal excitability polarity-dependently for up to $60 \mathrm{~min}$ after the end of the stimulation (Bailey et al., 2016). The first studies were conducted in the hand area of the motor cortex that showed corticospinal excitability alterations, monitored by transcranial magnetic stimulation (TMS)-induced motor evoked potentials (MEP) (Rossi et al., 2009), of up to $40 \%$. In the motor cortex, excitability enhancement was achieved by anodal stimulation, whereas cathodal stimulation reduced excitability (Nitsche and Paulus, 2000). Moreover, the strength and duration of these after-effects are controlled by current intensity and duration (Nitsche and Paulus, 2001; Nitsche et al., 2003b; MonteSilva et al., 2010, 2013; Batsikadze et al., 2013). Pharmacological studies (Liebetanz et al,, 2002; Nitsche et al., 2003a) identified a role of tDCS-induced membrane polarization and NMDA receptor activation for these sustained after-effects (Nitsche and Paulus, 2001).

Awareness about the relevance of computational modeling for rational design of electrode montages, taking into account not only the electric field strength but also the current flow direction in relation to neuronal orientation (Das et al., 2016), has increased recently. Computational modeling can help to identify optimal electrode positions, and improve efficacy of stimulation (Datta et al., 2011). In this study, we focused on the application of tDCS over the leg area of the motor cortex, which presents a challenge due to its depth and orientation in the interhemispheric fissure, and has not been explored as much as tDCS of the hand area of the motor cortex. Some studies, however, have shown that tDCS can modulate the excitability of the leg area of the motor cortex. Jeffery et al. (2007) showed that $10 \mathrm{~min}$ of stimulation with the anode over the leg area of the motor cortex in healthy humans increased corticospinal excitability of the anterior tibial (TA) muscle by up to $59 \%$ compared to baseline values for up to $60 \mathrm{~min}$ after stimulation. Cathodal tDCS, however, did not decrease corticospinal excitability. In a functional outcome study in healthy humans, anodal tDCS has been shown to transiently enhance maximal leg pinch force for up to $30 \mathrm{~min}$ after stimulation compared to baseline, but did not affect reaction time (Tanaka et al., 2009). Also here, cathodal tDCS did not alter performance. Roche et al. (2011) showed that anodal tDCS over the same area induced effects on spinal network excitability similar to those observed during co-contraction of lower-limb muscles. Such indirect effects on spinal network excitability may be suited to support postural stability and balance, as shown by the recent studies conducted in healthy humans (Dutta et al., 2014a; Kaminski et al., 2016).

Regarding clinical application of tDCS over the primary motor cortex leg area, anodal stimulation on the lesioned cortex with a large square sponge electrode $(5 \mathrm{~cm} \times 5 \mathrm{~cm})$ with $2 \mathrm{~mA}$ for 10 min improved balance and strengthened the affected lower limb in stroke patients (Sohn et al., 2013). Jayaram and Stinear (2009) explored the effects of anodal tDCS over the lesioned motor cortex of nine chronic stroke survivors using a small $8.1 \mathrm{~cm}^{2}$ saline-soaked sponge electrode as anode (unlike most other studies, which used relatively large $25-36 \mathrm{~cm}^{2}$ stimulation electrodes) whose edge was aligned to the midsagittal plane, and a large $36 \mathrm{~cm}^{2}$ cathode which was placed above the contralateral orbit. They investigated bilateral modulatory effects of stimulation on the tibialis anterior (TA), medial gastrocnemius, medial hamstrings, and vastus lateralis muscles. Anodal tDCS over the ipsilesional motor cortex increased paretic limb and decreased non-paretic limb motor excitability, and thus showed a relatively focal effect. Regarding effects on motor functions, a single session of anodal tDCS of the paretic lower limb was shown to increase knee extensor force for up to $30 \mathrm{~min}$ following stimulation in hemiparetic stroke survivors (Tanaka et al., 2011). van Asseldonk and Boonstra (2016) showed similar montage-related performance differences in 10 healthy subjects and 10 chronic stroke survivors that also revealed a large inter-individual variability of effects. In that study, two montages with a $5 \mathrm{~cm} \times 7 \mathrm{~cm}$ anode placed over the lesioned hemisphere with the short edge of the rectangular electrode aligned to the mid-sagittal fissure and centered over the motor cortex representation of the leg, and the cathode placed over the supraorbital region (called unihemispheric montage) or over the motor cortex contralateral to the targeted area (called bihemispheric montage) were compared. In the study of van Asseldonk and Boonstra (2016), subjects with the largest effect for one montage often showed opposite effects for the other. This underscores the relevance of the placement of the electrodes when aiming to stimulate the leg area, analogous to what has been described for the hand area (Bikson et al., 2010; Moliadze et al., 2010). Placement of the electrodes is not only critical for the electric field strength, but also electric field direction (Rawji et al., 2018). Both factors are relevant for stimulation of the leg area of the motor cortex due to its depth and orientation in the inter-hemispheric fissure. However, a comprehensive finite element modeling of tDCS of the leg motor area with a realistic head model and physiological validation of the computational results has not been conducted so far. Stimulation parameters and brain anatomy affect efficacy and specificity of tDCS, which is particularly challenging for cortical areas not on the brain surface such as the leg area of the motor cortex.

In our preliminary study (Dutta et al., 2012) using a simple three-shell head model, we hypothesized that not only the electric field strength but also the electric field direction is relevant for the effects of anodal tDCS over the leg motor area. For the present study, our goal was to maximally stimulate the targeted leg motor representation while avoiding stimulation of the contralateral leg motor volume. We investigated simple two-electrode unihemispheric montages using a realistic computational head model and explored the impact of cathode placement and anode size on anodal tDCS over the motor cortex leg area. We then evaluated the appropriateness of the computational models via neurophysiological testing in healthy individuals. 


\section{MATERIALS AND METHODS}

\section{Finite Element Model of the Human Head}

The head model for finite element modeling was developed using the freely available SimNIBS software pipeline. ${ }^{1}$ The SimNIBS software pipeline (Windhoff et al., 2013) uses fat-suppressed T1-weighted magnetic resonance images (MRI) as input for FreeSurfer (Fischl, 2012). We used the Colin27 average brain (Collins et al., 1998; Holmes et al., 1998), which is the stereotaxic average of 27 T1-weighted MRI scans of the same individual, to create the head model (see iso2mesh toolbox (iso2mesh; Fang and Boas, 2009). The Colin27 average brain has been widely adopted as a stereotaxic template that includes and labels cerebellum, brain stem, and ventricles. After segmentation, different components like scalp, skull, cerebrospinal fluid (CSF), white matter (WM), and gray matter (GM) of the brain were modeled as different volume conductors with their own specific conductivity (Windhoff et al., 2013), as shown in Table $\mathbf{1}$.

The anode and cathode injected a specified amount of current (source) in the volume conductor. The electrodes were modeled as saline-soaked sponge cuboids (see section "Electrode Montages for Finite Element Modeling"). We analyzed the headmodel for electric field distribution using the Finite Element Method (FEM), provided in the SimNIBS pipeline, which provides a powerful numerical tool to solve the required partial differential equations (PDE).

The quasi-static formula for direct current stimulation is given below,

$$
-\nabla \cdot(\sigma \nabla V)=\sin \Omega
$$

where $\Omega$ is the volume conductor, $V_{(x, y, z)}$ is the scalar potential field, $\sigma_{(x, y, z)}$ is the conductivity tensor, $S$ is the source term. The Dirichlet boundary condition is presented in Section "Electrode Montages for Finite Element Modeling". FEM divides the volume conductor into spatial elements and nodes for discrete computations of the PDE. The tetrahedral head meshes for FEM were generated using the "mri2mesh" tool in the SimNIBS software pipeline (Windhoff et al., 2013) with an average tetrahedron volume of $1 \mathrm{~mm}^{3}$. The continuity of the solution is maintained at the boundary of the elements using shape function objects. The electric field values at the nodes within the bilateral leg area cluster in the cortical tissue (not CSF) were captured by Boolean intersection with a sphere of $1 \mathrm{~cm}$ radius centered at $(-7 \mathrm{~mm},-38 \mathrm{~mm}, 75 \mathrm{~mm})$ and $(6 \mathrm{~mm},-38 \mathrm{~mm}, 75 \mathrm{~mm})$ in the MNI coordinates, as shown in Figure 1. The cortical tissue

${ }^{1}$ www.simnibs.org

\section{TABLE 1| Electrical conductivity.}

\begin{tabular}{lc}
\hline Component & Electrical conductivity $\left(\mathbf{S ~ m}^{-1}\right)$ \\
\hline Scalp & 0.465 \\
Skull & 0.010 \\
CSF & 1.654 \\
Gray matter & 0.276 \\
White matter & 0.126
\end{tabular}

cluster after Boolean intersection with the sphere was comparable to the functional MRI activation volume $\left(\sim 1450 \mathrm{~mm}^{3}\right)$ during plantar $\left(45^{\circ}\right)$ and dorsal flexion $\left(10^{\circ}\right)$ of the foot at a rate of approximately $0.5 \mathrm{~Hz}$ (Alkadhi et al., 2002). All node values within the cortical tissue clusters were imported in Matlab (The Mathworks, Inc., United States) to compute the average magnitude and direction (described in section "Optimization of Electrode Montage").

\section{Electrode Montages for Finite Element \\ Modeling}

The electrode positions were defined with fiducials at $\mathrm{Nz}, \mathrm{Iz}$, right, and left preauricular points for registration with the head model in accordance with the 10-10 system defined in Oostenveld and Praamstra (2001). We explored the effects of the following electrode positions, and sizes: motor cortex anode (large: $5 \mathrm{~cm} \times 7 \mathrm{~cm}$ at $2 \mathrm{~mA}$ and small: $3.5 \mathrm{~cm} \times 1 \mathrm{~cm}$ at $0.2 \mathrm{~mA}$ ) at the approximate TA muscle hotspot based on neurophysiological testing (Dutta et al., 2014b) - $15 \mathrm{~mm}$ left lateral and $20 \mathrm{~mm}$ posterior to $\mathrm{Cz}(1 \mathrm{~mm},-28 \mathrm{~mm}, 87 \mathrm{~mm})$. The cathode $(5 \mathrm{~cm} \times 7 \mathrm{~cm})$ was placed at Fp1 $(-21 \mathrm{~mm}, 70 \mathrm{~mm}, 15 \mathrm{~mm})$, F7 $(-53 \mathrm{~mm}, 32 \mathrm{~mm}, 2 \mathrm{~mm})$, T7 $(-70 \mathrm{~mm},-16 \mathrm{~mm},-8 \mathrm{~mm})$, P7 $(-58 \mathrm{~mm},-65 \mathrm{~mm},-6 \mathrm{~mm}), \mathrm{Oz}(1 \mathrm{~mm},-101 \mathrm{~mm}, 6 \mathrm{~mm})$, P8 $(56 \mathrm{~mm},-64 \mathrm{~mm},-6 \mathrm{~mm}), \mathrm{T} 8(55 \mathrm{~mm}, 30 \mathrm{~mm},-1 \mathrm{~mm})$, and Fp2 $(25 \mathrm{~mm}, 68 \mathrm{~mm}, 15 \mathrm{~mm})$. Here, $(x, y$, and $z)$ refer to the MNI stereotaxic space (Jurcak et al., 2007); the $x$ direction is mediolateral, the $y$ direction anterior-posterior, and the $z$ direction ventro-dorsal. This resulted in eight montages with cortical projection of their respective electrode center denoted using the 10-10 EEG system (Koessler et al., 2009). The contralateral supraorbital cathode position (Fp2) was termed "conventional montage," since this montage was most often used in prior tDCS studies of the leg motor area (Madhavan and Shah, 2012). These eight electrode montages were evaluated computationally, as described in Section "Optimization of Electrode Montage," based on the Colin27 FEM head model (see section "Finite Element Model of the Human Head"). Transcranially injected direct current per unit area at the top of the saline-soaked sponge anode was set constant at $0.057 \mathrm{~mA} / \mathrm{cm}^{2}$ which was a Dirichlet boundary condition for the FEM head model.

\section{Optimization of Electrode Montage}

The tDCS current per unit area at the top of the sponge electrodes was kept constant at $0.057 \mathrm{~mA} / \mathrm{cm}^{2}$ for the computational optimization of the electrode montage that resulted in $2 \mathrm{~mA}$ direct current for the large-anode and $0.2 \mathrm{~mA}$ for the smallanode. This current amplitude is considered to be safe and adequate for experimental validation in healthy humans (Nitsche et al., 2003b). The electric field values (see section "Finite Element Model of the Human Head") at the nodes within the bilateral leg motor volume were extracted with the "CutSphere" command of $\mathrm{Gmsh}^{2}$ (Geuzaine and Remacle, 2009) and imported in Matlab (The Mathworks Inc., United States) as a text file for computing their average magnitude and direction. Here, the 
A Targeted motor cortex sphere centered at $(-7 \mathrm{~mm},-38 \mathrm{~mm}, 75 \mathrm{~mm})$ in the MNI coordinates
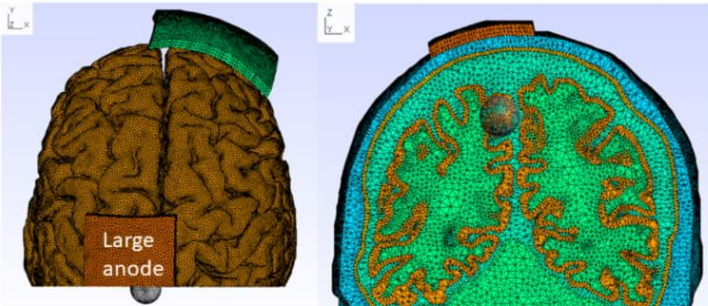

B Contralateral motorcortex sphere centered at $(6 \mathrm{~mm},-38 \mathrm{~mm}, 75 \mathrm{~mm})$ in the $\mathrm{MNI}$ coordinates
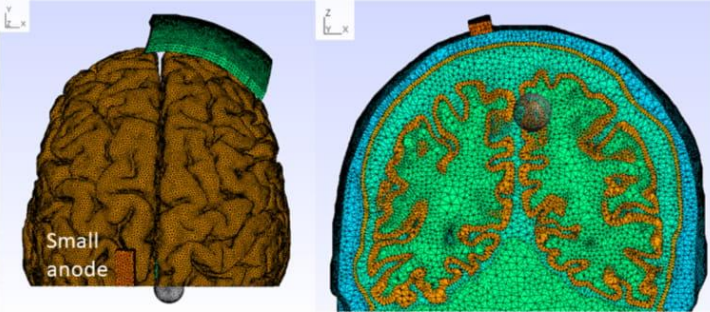

FIGURE 1 | Colin27 FEM head model for the "conventional montage" electrode arrangement with the cathode $(5 \mathrm{~cm} \times 7 \mathrm{~cm})$ at Fp2 (10-10 EEG system) and anode centered at $15 \mathrm{~mm}$ left lateral and $20 \mathrm{~mm}$ posterior to $\mathrm{Cz}$ (10-10 EEG system). (A) Large-anode: $5 \mathrm{~cm} \times 7 \mathrm{~cm}$ at $2 \mathrm{~mA}$, (B) Small-anode: $3.5 \mathrm{~cm} \times 1 \mathrm{~cm}$ at $0.2 \mathrm{~mA}$. The cortical tissue cluster found after Boolean intersection with the sphere of a radius of $1 \mathrm{~cm}$ captures the electric field at the targeted [centered at $(-7 \mathrm{~mm}$, $-38 \mathrm{~mm}, 75 \mathrm{~mm}) \mathrm{MNI}$ coordinates - A] and the contralateral [centered at $(6 \mathrm{~mm},-38 \mathrm{~mm}, 75 \mathrm{~mm}) \mathrm{MNI}$ coordinates - B] leg motor cortex, as shown with top (left panel) and coronal (right panel) sectional views.

cortical tissue cluster found after Boolean intersection of the cortical tissue with the sphere of a radius of $1 \mathrm{~cm}$ with centroids at $(-7 \mathrm{~mm},-38 \mathrm{~mm}, 75 \mathrm{~mm})$ and $(6 \mathrm{~mm},-38 \mathrm{~mm}, 75 \mathrm{~mm})$ in MNI coordinates (see Figure 1) represented the targeted and contralateral leg motor volume respectively. The specificity of the electric field $\underset{E F}{\rightarrow})$ for different cathode locations (Fp1, F7, T7, P7, $\mathrm{Oz}, \mathrm{P} 8, \mathrm{~T} 6, \mathrm{~T} 8$, and Fp2) was determined by the laterality of the volume-averaged electric field strength (Opitz et al., 2015) toward the targeted leg motor volume. Therefore, the specificity was computationally (comp) found based on the volume-averaged magnitude of the electric field $(|E F|=\sqrt{E F o E F})$ or volumeaveraged electric field strength (Opitz et al., 2015),

$$
\text { Specificity }_{\text {comp }}^{\text {montage }}=\frac{\left(|E F|_{\text {targeted }}-|E F|_{\text {contralateral }}\right)}{\left(|E F|_{\text {targeted }}+|E F|_{\text {contralateral }}\right)}
$$

The best montage based on the computational (comp) analysis, Specificity comp , was compared with the "conventional montage" based on neurophysiological testing (see section "Experimental Validation"). Our goal was to maximally stimulate the targeted leg motor volume [centroid at $(-7 \mathrm{~mm},-38 \mathrm{~mm}$, $75 \mathrm{~mm})$ MNI coordinates] while avoiding stimulation of the contralateral leg motor volume [centroid at $(6 \mathrm{~mm},-38 \mathrm{~mm}$, $75 \mathrm{~mm}$ ) MNI coordinates] - see Figure 1. The volume-averaged electric field $(\rightarrow)$ unit vector was also computed for the targeted (targ in Equation 2b) and contralateral (contra in Equation 2b) leg motor volumes, and the angle between these vectors was used for comparison

$$
\text { Angle }_{\text {comp }}^{\text {montage }}=\angle \overrightarrow{E F}_{\text {targeted }}-\angle \overrightarrow{E F}_{\text {contralateral }}
$$

\section{Experimental Validation}

Twelve healthy subjects, seven males and five females (age: 21-36 years, all right-leg dominant) volunteered for the study. The subjects signed an informed consent form before participation and the study was approved by the Institutional Review Board of the University Medical Center, Goettingen, Germany. The experiment consisted of multiple sessions of anodal or sham tDCS with each session addressing a separate electrode montage (list given in Table 2, complete cross-over design) in randomized order, with sufficient (1 week) "wash-out" time in between the sessions.

The anode was placed over the dominant right-leg motor cortex representation, as shown in Figure 1. Figure 1 also shows the targeted and contralateral leg motor volumes, which were used to compute the specificity of the stimulation. A transcranial DC stimulator (NeuroConn, Germany) delivered the currents for $10 \mathrm{~min}$ via the anode centered on the scalp at the position where TMS of the primary motor cortex elicited maximal twitches in the resting dominant right-leg TA muscle. TMS was delivered with a Magpro Stimulator (MagVenture, United States) through a butterfly coil (MC-B70, MagVenture, 
TABLE 2 | Electrode montages and stimulation parameters for neurophysiological testing.

\begin{tabular}{lcc}
\hline Montage & Anode & Cathode \\
\hline $\begin{array}{l}\text { Large-anode in } \\
\text { conventional montage }\end{array}$ & $5 \mathrm{~cm} \times 7 \mathrm{~cm}$ at $2 \mathrm{~mA}$ & $5 \mathrm{~cm} \times 7 \mathrm{~cm}$ at FP2 \\
$\begin{array}{l}\text { Small-anode in } \\
\text { conventional montage }\end{array}$ & $3.5 \mathrm{~cm} \times 1 \mathrm{~cm}$ at $0.2 \mathrm{~mA}$ & $5 \mathrm{~cm} \times 7 \mathrm{~cm}$ at FP2 \\
$\begin{array}{l}\text { Small-anode in side } \\
\text { montage }\end{array}$ & $3.5 \mathrm{~cm} \times 1 \mathrm{~cm}$ at $0.2 \mathrm{~mA}$ & $5 \mathrm{~cm} \times 7 \mathrm{~cm}$ at T7 \\
$\begin{array}{l}\text { Small-anode in sham } \\
\text { montage }\end{array}$ & $3.5 \mathrm{~cm} \times 1 \mathrm{~cm}$ at $0 \mathrm{~mA}$ & $5 \mathrm{~cm} \times 7 \mathrm{~cm}$ at FP2 \\
& & \\
\end{tabular}

United States) and the resting muscle activity as well as the MEP were monitored using biofeedback software (Signal 2 software, CED, United Kingdom). For TMS of the right-leg motor area, a right-to-left oriented current flow in the brain tissue is required for MEP generation and conversely, when stimulating the left-leg motor area with TMS, a left-to-right oriented current is optimal. The handle of the TMS butterfly coil was thus aligned approximately $90^{\circ}$ to the parasagittal plane to induce a tissue current that runs in the coronal plane in the required direction (Groppa et al., 2012). The location of the coil on the scalp for the targeted rightleg, called the "target-hotspot," was identified with singlepulse TMS by adjusting the coil position until it resulted in the largest MEP at a moderate suprathreshold stimulation intensity. Then, the contralateral left-leg hotspot, called the "contralateral hotspot," was identified. Both hotspots were marked with water-resistant ink to reduce variability of coil placement during bilateral testing of corticospinal excitability. Corticospinal excitability alterations (Rossini et al., 1999) were evaluated using single-pulse TMS intensity that elicited 10 MEPs of average $0.5 \mathrm{mV}$ amplitude at baseline before intervention. Corticospinal excitability was monitored at the "target-hotspot" as well as the "contralateral-hotspot." Corticospinal excitability was measured before and immediately after the completion of tDCS as well as every $15 \mathrm{~min}$ for the next $60 \mathrm{~min}$, and then every $30 \mathrm{~min}$ for next $60 \mathrm{~min}$ for each session, and $24 \mathrm{~h}$ for the real stimulation conditions, 10 MEPs were recorded for each time bin. For sham tDCS, the current was ramped up for $15 \mathrm{~s}$ and then down to zero in $15 \mathrm{~s}$ for blinding purposes. All subjects included in this study responded at baseline to single-pulse TMS with 10 MEPs of an average $0.5 \mathrm{mV}$ at the "target-hotspot" as well as at the "contralateralhotspot."

During anodal tDCS of the "target-hotspot," the current was ramped up linearly for $15 \mathrm{~s}$ to a constant amplitude of either 2 or $0.2 \mathrm{~mA}$ which was maintained for $10 \mathrm{~min}$ before being ramped down linearly for $15 \mathrm{~s}$.

The specificity of the corticospinal excitability after-effects based on MEP-based neurophysiological (neurophys) measures at the "target-hotspot" and the "contralateral-hotspot" was computed as,

$$
\text { Specificity }_{\text {neurophys }}^{\text {montage }}=\frac{\left(M E P_{\text {targeted }}-M E P_{\text {contralateral }}\right)}{\left(M E P_{\text {targeted }}+M E P_{\text {contralateral }}\right)}
$$

Here, $M E P_{\text {targeted }}$ is the MEP-based measure of corticospinal excitability at the "target-hotspot" and the $M E P_{\text {contralateral }}$ is the one at the "contralateral-hotspot."

Two-way repeated measure ANOVAs (within subject factors: time post-tDCS and tDCS-condition, dependent variables: baseline-normalized MEP and Specificity montage electrophys $_{\text {) }}$ were conducted to calculate the effect of the tDCS-conditions: large-anode in the "conventional montage," small-anode in the "conventional montage," small-anode in the "side montage," and small-anode in the "sham montage." Pairwise post hoc comparisons were carried out using $t$-statistics with Bonferroni correction ("multcompare" in Matlab). Alpha was set at $P<0.05$.

\section{RESULTS}

The results from the computational modeling of the electric field at the targeted right-leg motor volume [centroid at $(-7 \mathrm{~mm}$, $-38 \mathrm{~mm}, 75 \mathrm{~mm}$ ) in MNI coordinates] and the contralateral left-leg motor volume [centroid at $(6 \mathrm{~mm},-38 \mathrm{~mm}, 75 \mathrm{~mm})$ in MNI coordinates] are shown in Figure 2. The maximum electric field magnitude at the targeted leg motor volume for the largeanode, $5 \mathrm{~cm} \times 7 \mathrm{~cm}$, at $2 \mathrm{~mA}$, was around $0.4 \mathrm{~V} / \mathrm{m}$, while for the small-anode, $3.5 \mathrm{~cm} \times 1 \mathrm{~cm}$ at $0.2 \mathrm{~mA}$, it was around $0.05 \mathrm{~V} / \mathrm{m}$. Therefore, the maximum electric field strength was about onetenth at the targeted right-leg motor volume with the smallanode (Figures 2B,D) when compared to that for the large-anode (Figures 2A,C). For the small-anode, the maximum electric field strength was found to be higher at the targeted right-leg motor volume than the contralateral left-leg motor volume with the cathode at T7 (Figure 2D) when compared to the cathode at Fp2 (Figure 2B). This difference in the electric field strength was captured with the specificity metric from finite element analysis. The Specificitycomp for the large-anode (in black) and small-anode (in gray) for different cathode locations is shown in Figure 3A. The T7 cathode location provided the best specificity for both the large-anode and the small-anode. This best montage identified by computational analysis with the smallanode positioned over the "target-hotspot" and the cathode over T7 was labeled "side montage" for neurophysiological testing. Also, the angle between the average electric field direction (unit vector) at the targeted right-leg and the contralateral left-leg motor volume, Angle montage are shown in Figure 3B. The small-anode resulted in a larger Angle comp $^{\text {moge }}$ when compared to the large-anode, however the distribution across cathode locations, Fp1, F7, T7, P7, Oz, P8, T6, and Fp2 (10-10 EEG system) was similar for the small-anode and the large-anode.

For neurophysiological evaluation based on Specificity $\begin{gathered}\text { montage } \\ \text { neurophys }\end{gathered}=\frac{\left(M E P_{\text {targeted }}-M E P_{\text {contralateral }}\right)}{\left(M E P_{\text {tage }}+M E P_{\text {contal }}\right)}$, the "side montage" was compared with the "conventional montage."

Figure 4 shows the results from the neurophysiological testing of corticospinal excitability changes following anodal tDCS. All results are displayed as mean \pm standard error of means. The corticospinal excitability changes are presented as MEPs individually normalized to baseline (baseline-normalized 


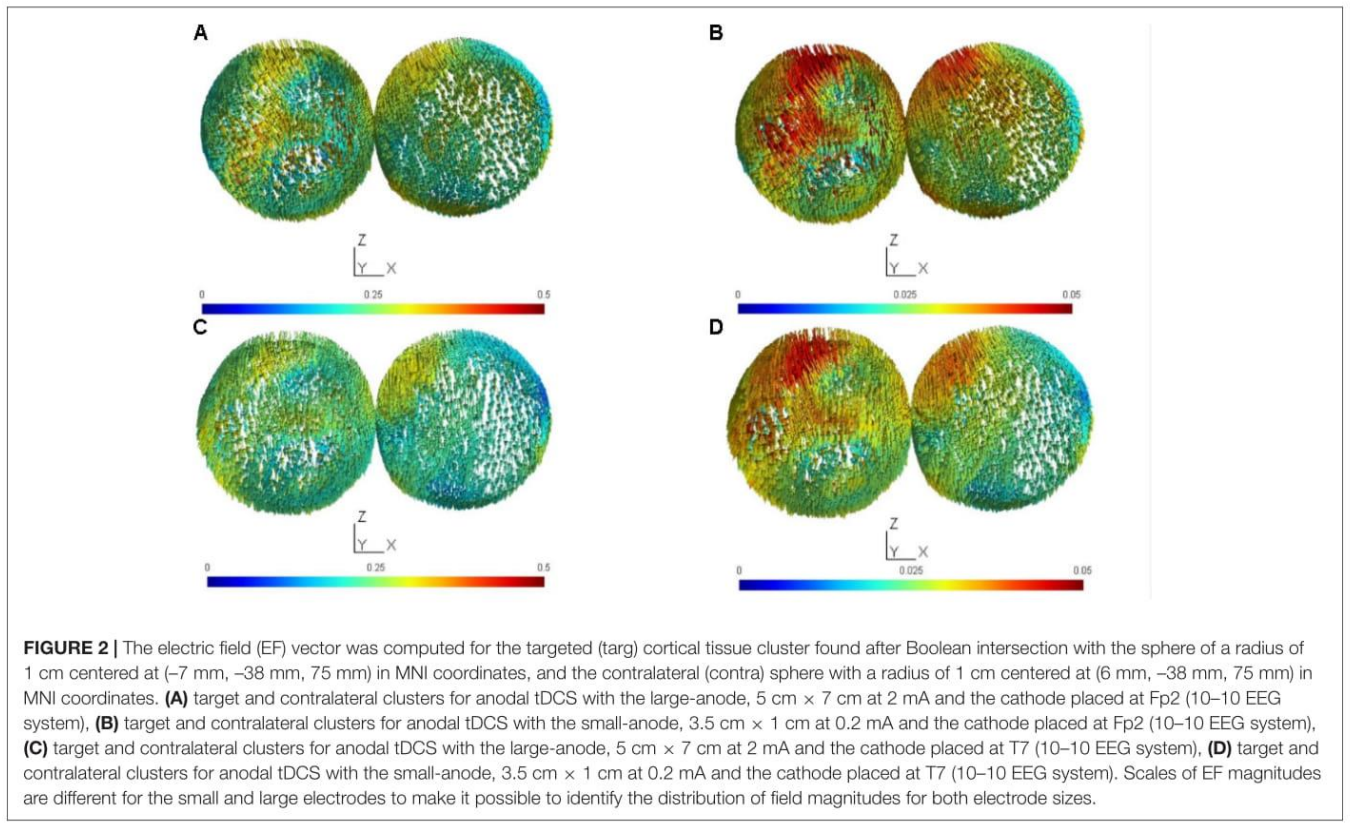

MEP) from the targeted right-leg and the contralateral leftleg TA muscles before and immediately after the completion of anodal tDCS as well as every $15 \mathrm{~min}$ for the next $60 \mathrm{~min}$, and then every $30 \mathrm{~min}$ for the next $60 \mathrm{~min}$ for each session. The repeated measure two-way ANOVA [within subject factors: time post-tDCS(min): $0,15,30,45,60,90,120$, and tDCScondition: large-anode "conventional montage" small-anode "conventional montage," small-anode "side montage," smallanode "sham montage"] conducted for the dependent variable baseline-normalized MEP of the right-leg "target-hotspot" showed significant main effects of time post-tDCS $[F(6)=4.65$, $P<0.05]$ and tDCS-condition $[F(3)=23.44, P<0.05]$, but no significant interaction $[F(18)=1.18, P=0.264]$. For the dependent variable baseline-normalized MEP of the left-leg "contralateral-hotspot," a significant main effect was found only for tDCS-condition $[\mathrm{F}(3)=9.79, \mathrm{P}<0.05]$ but not for time $[F(6)=2.08, P=0.0528]$ or the respective interaction $[F(18)=0.6$, $P=0.9011]$

The Specificity $\begin{gathered}\text { montage } \\ \text { electrophys }\end{gathered}$ effects for different tDCS conditions is shown in Figure 5 for the single subject level. All results are displayed as mean \pm standard error of means. The post hoc tests using $t$-statistics with Bonferroni correction revealed that the baselinenormalized MEP of the right-leg "target-hotspot" for the small-anode in the "sham montage" was lowest and differed significantly $(P<0.05)$ from the other tDCS-conditions after intervention (Figure 6A). The baseline-normalized MEP of the left-leg "contralateral-hotspot" were highest for the large-anode "conventional montage," and differed significantly $(P<0.05)$ from the other tDCS-conditions (Figure 6B). Consequently, Specificity montage of the corticospinal excitability after-effects, which is the normalized difference between the baselinenormalized MEP of the right-leg "target-hotspot" and the left-leg "contralateral-hotspot" was found to be negative (95\% confidence interval) for the large-anode "conventional montage" in the post hoc tests (see Figure 6C). Post hoc tests revealed that the Specificity $\begin{aligned} & \text { montage } \\ & \text { neurophys }\end{aligned}$ was significantly different $(P<0.05)$ for different tDCS-conditions, with the small-anode "side montage" having the highest mean (i.e., best montage based on Specificity $\left.\begin{array}{c}\text { montage } \\ \text { neurophys }\end{array}\right)$, followed by the small-anode "conventional montage," the large-anode "conventional montage," and then the small-anode "sham montage" - see Figure 6C.

\section{DISCUSSION}

The results of this study supply information about the effects of electrode montage and anode size on the specificity of anodal tDCS after-effects on the leg motor area. All active stimulation conditions induced the expected target motor cortex excitability enhancements. Hereby, the small-anode "side montage" configuration, i.e., $3.5 \mathrm{~cm} \times 1 \mathrm{~cm}$ anode placed over the right-leg motor "target-hotspot" with the cathode placed over T7 (10-10 EEG system) was found to be superior to both "conventional montages" with the cathode positioned over Fp2 (10-10 EEG system) in terms of specificity in both the 

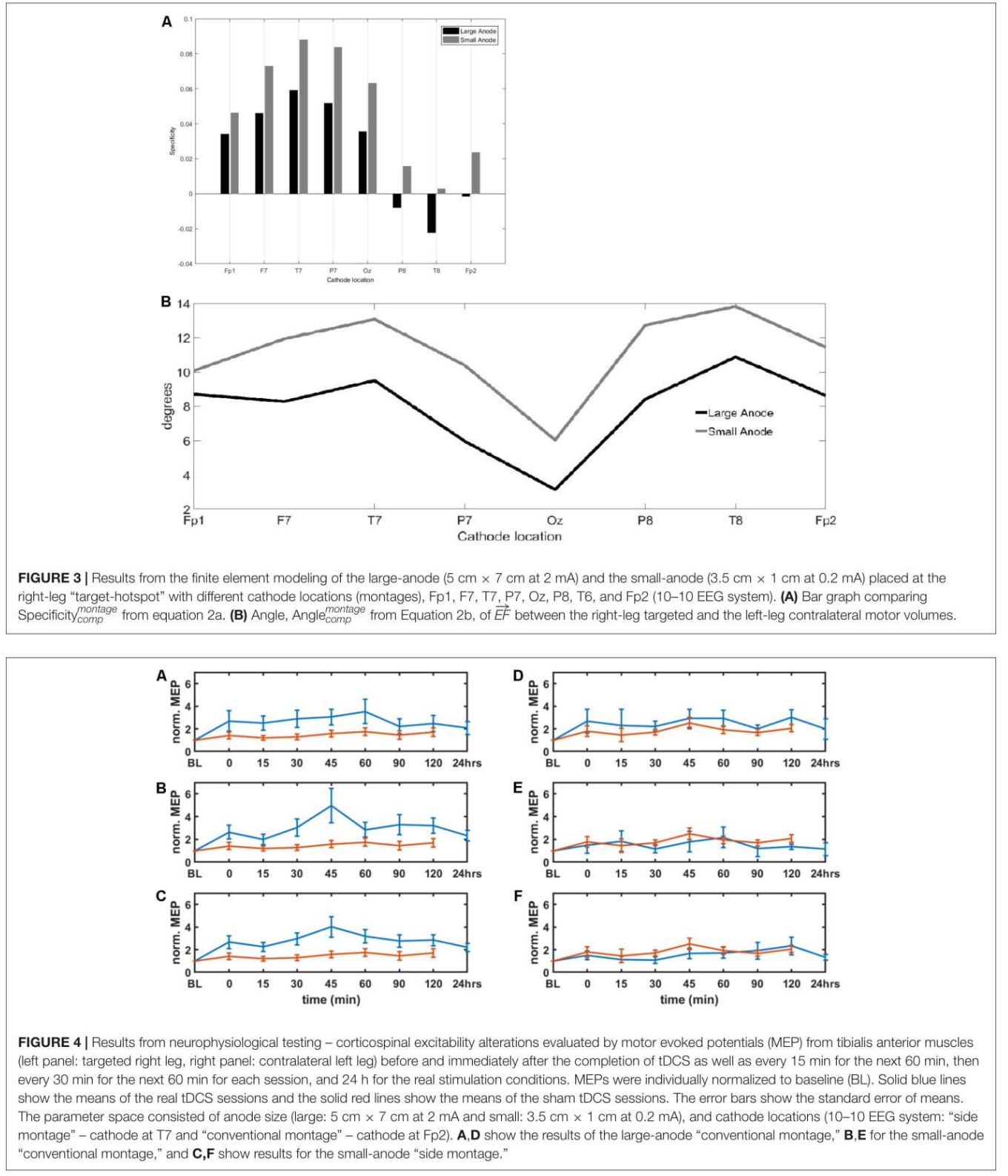

computational analysis (Figure 3) and neurophysiological testing (Figure 6C). The simulated maximum electric field strength was about one-tenth at the targeted right-leg motor volume with the small-anode (Figures 2B,D), as compared to that induced by the large-anode (Figures 2A,C). Nevertheless, the small-anode montage altered cortical excitability, in agreement 

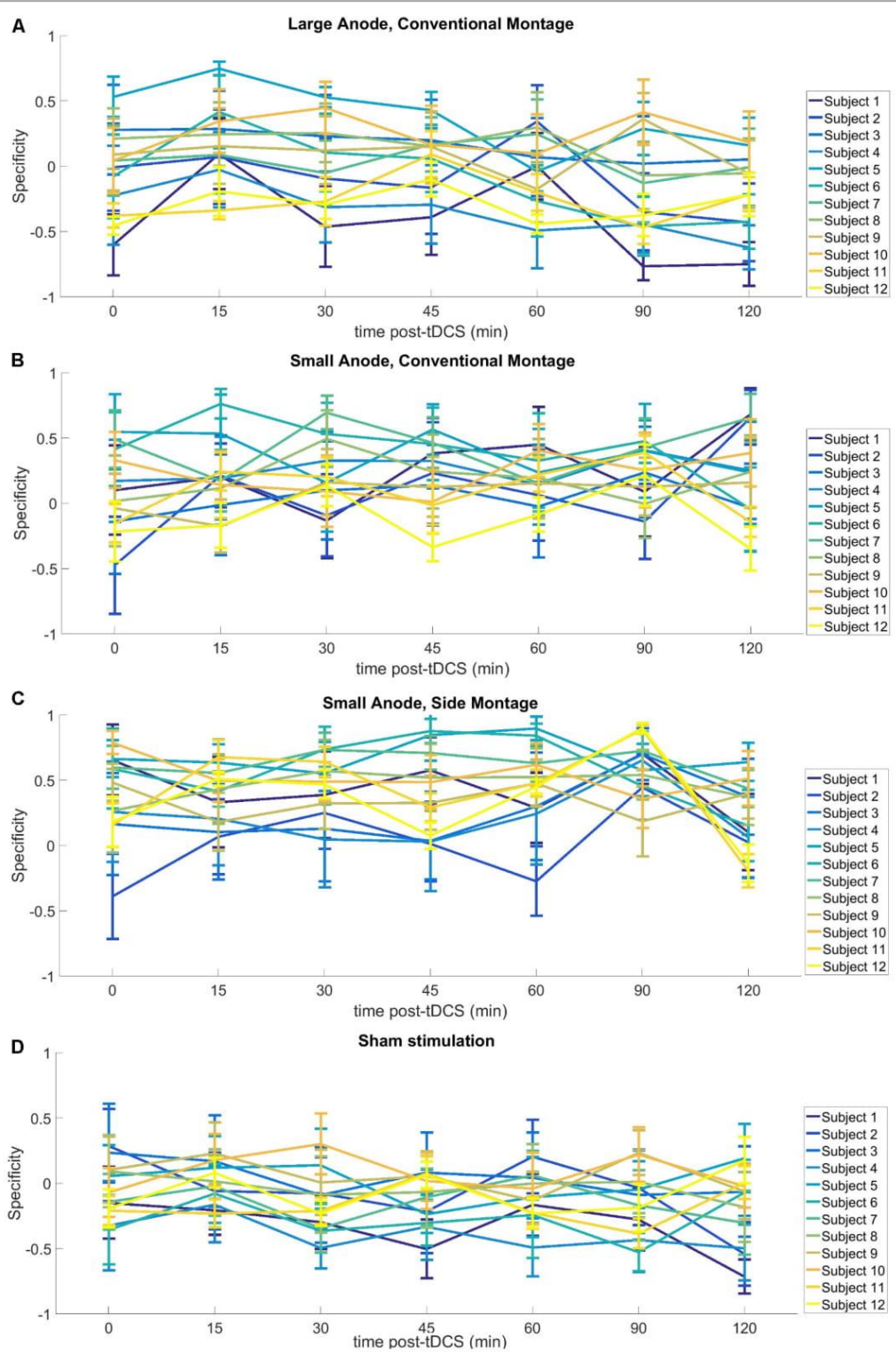

FIGURE $\mathbf{5}$ | Results from neurophysiological testing - Specificity montage - of the corticospinal excitability after-effects for different tDCS-conditions with regard to interindividual variability, (A) large-anode, "conventional montage": large anode, $5 \mathrm{~cm} \times 7 \mathrm{~cm}$ at $2 \mathrm{~mA}$, over the right-leg "target-hotspot" with the cathode over Fp2 (10-10 FFG system), (B) small-anode, "conventional montage": small anode, $3.5 \mathrm{~cm} \times 1 \mathrm{~cm}$ at $0.2 \mathrm{~mA}$, over right-leg "target-hotspot" with the cathode over Fp2 (10-10 EEG system), (C) small anode, "side montage": small-anode, $3.5 \mathrm{~cm} \times 1 \mathrm{~cm}$ at $0.2 \mathrm{~mA}$, over right-leg "target-hotspot" with the cathode over T7 (10-10 EEG system), (D) Sham stimulation: large anode, $5 \mathrm{~cm} \times 7 \mathrm{~cm}$ at $2 \mathrm{~mA}$, at right-leg "target-hotspot" with the cathode at Fp2 (10-10 EEG system). Here, relatively large individual variability is notable, including the sham montage. 

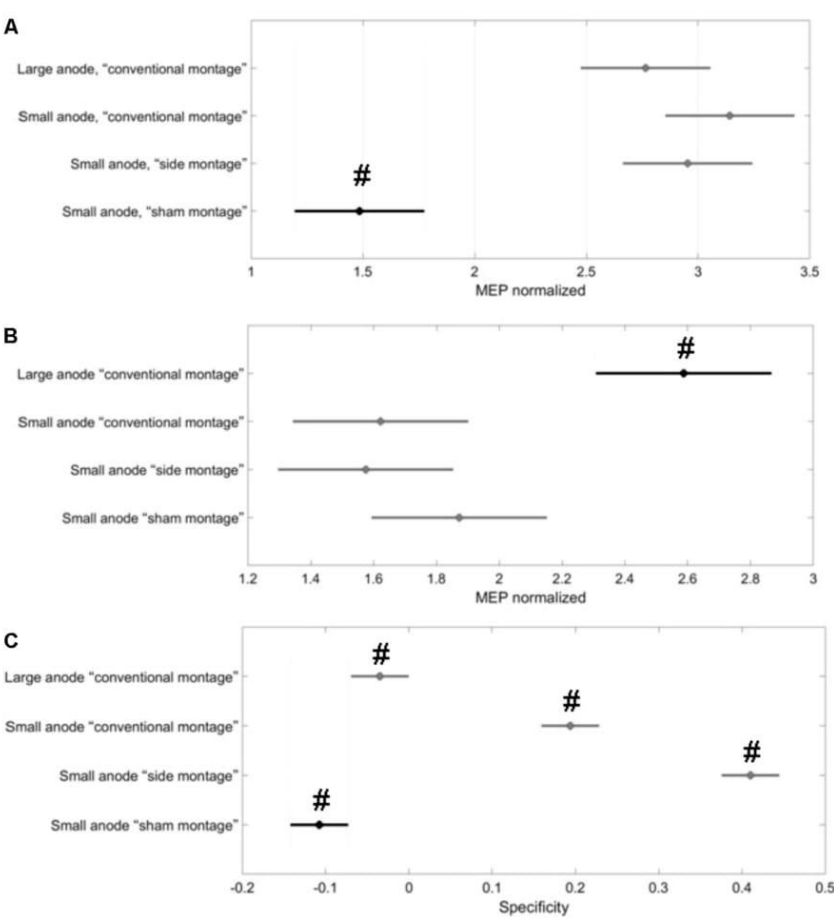

FIGURE 6 | Results from the post hoc tests $(P<0.05)$ presented with $95 \%$ confidence intervals generated from all MEPs. Black represents the data contrasted in the respective post hoc comparison with the other conditions (in gray). Here, two group means are significantly different if their intervals are disjoint; they do not differ significantly if their intervals overlap. (A) The baseline-normalized MEP of the right-leg "target-hotspot" was lowest and significantly different (marked with \#) in the "sham montage" when compared to all real tDCS conditions. (B) the baseline-normalized MEP of the left-leg "contralateral-hotspot" was largest for the large-anode "conventional montage," which differed significantly (marked with \#) from all other tDCS-conditions. (C) Specificity elentage $^{\text {monohy }}$ values were significantly different from each other for all tDCS-conditions (marked with \#), with the small-anode "side montage" having the highest mean, then the small-anode "conventional montage," followed by large-anode "conventional montage" and then, the small-anode "sham montage.

with prior works (Madhavan and Stinear, 2010), in both the "conventional montage" (Figures 4B,E) as well as "side montage" (Figures 4C,F). Therefore, the physiological effects over this target region did not correlate linearly with simulated electrical field (EF) strength. This finding is in accordance with those of a recent study, where it was shown that for a relatively large range of stimulation intensities, anodal tDCS over the motor cortex resulted in similar MEP alterations (Jamil et al., 2017), thus physiological effects may not scale linearly with electric field strength. Alternatively, it cannot be ruled out that the currently available models do not deliver sufficiently correct simulations of EF strength.

In this study, in contrast to the large-anode "conventional montage," the small-anode electrode arrangements resulted in a positive Specificity $\begin{gathered}\text { montage } \\ \text { in the computational analysis for the }\end{gathered}$ "conventional montage" as well as the "side montage," which was confirmed by neurophysiological testing of the Specificity montage
Here, Specificitycomp $\begin{gathered}\text { montage } \\ \text { com }\end{gathered}$ was defined based on the volume averaged magnitude of the electric field or volume-averaged electric field strength (Opitz et al., 2015). Neurophysiological testing confirmed in concurrence with the computational analysis that the small-anode "side montage" provided the best specificity across all evaluated tDCS-conditions: large-anode "conventional montage," small-anode "conventional montage," small-anode "side montage," and small-anode "sham montage." Beyond EF strength that was used to define Specificity montage $_{\text {comp }}$, directionality of the current flow might have relevantly contributed to the specificity differences between electrode arrangements. We found from Figure 3B that tDCS cathode locations over F7, T7, and $\mathrm{P} 7$, with the anode over the left primary motor cortex resulted in $\overrightarrow{E F}$ that was primarily in the right-to-left direction in the coronal plane at the right-leg "target-hotspot." This is in accordance with the respective TMS results (Priori et al., 1993) showing that the threshold is lowest for MEPs in the right-leg TA muscle when 
the current in the TMS coil flows from the left to the right side in the coronal plane, i.e, right-to-left oriented induced current in the right-leg "target-hotspot." Figure $3 \mathbf{B}$ shows that the EF direction differs on an average by $11.5^{\circ}$ for the small anode and $9^{\circ}$ for the large anode for the targeted and contralateral ROIs of the "conventional montage" (Fp2 cathode location). Here, the electric field direction is primarily posterior-anterior (PA) (rather than medio-lateral) in the "conventional montage" (Fp2 cathode location). Neurophysiological results showed that MEPs increased comparably in all real tDCS conditions for the targeted leg when compared to sham (see Figure 6A for the targeted leg). However, for the contralateral leg, only the large anode in the conventional montage resulted in a significant increase of MEP as compared to sham stimulation, as shown in Figure 6B. The relatively small difference in EF directions and higher magnitude of the electric field in the large anode conventional montage design, which covers a large volume of the brain including contralateral M1 (caused by the distant anterior position of the return electrode in the "conventional montage") can explain the identically directed effects of stimulation at the targeted and contralateral M1 in this condition. The relatively high magnitude of the EF in this condition $(\sim 0.4 \mathrm{~V} / \mathrm{m}$ with the large anode - see Figure 2) should be sufficient to affect M1 bilaterally. The relatively small difference of EF directions in the right and left motor cortices, most probably caused by the long-distant anterior position of the return electrodes, explains the identically directed effects of stimulation with the large electrodes on both areas, taking also into account that tDCS does not have an effect only on pyramidal neurons, but also on interneurons, which might be directed relevantly in AP/PA directions (Nitsche et al., 2005). The differences between the results of the small and large electrodes with the Fp2 return electrode positions, which resulted in similarly oriented EF vectors, and roughly comparable ipsi- and contralateral EF strength, are most probably caused by different specificity values, as shown by the results of the modeling, where the small electrode resulted in higher specificity in favor of the targeted motor cortex as compared to the large electrode, which resulted in zero specificity. Moreover, the lower absolute EF strength generated by the small electrode according to the modeling results, might have contributed, taking into account that a critical EF strength is assumed to exist, below which no excitability alterations are expected. The specific foundations for these results should, however, be further explored in future studies.

Some limitations of the study should be taken into account. The SimNIBS automated software pipeline (Windhoff et al., 2013) used in this study for computational modeling did not use a subject-specific head model. Therefore, the accuracy of the computed values is limited by the dimensions, the tissues modeled, and the isotropic conductivity values selected for the volume conduction head model. Thus whereas relations between different electrode configurations and placements should be relatively reliable, exact numerical results should be treated with caution. Nevertheless, such simple head models may increase our understanding of how stimulation parameters affect the electric field distribution. For example, Faria et al. (2011) showed that the magnitude of the current density falls more rapidly for smaller electrodes so one will need a higher current density at the electrode to get the same current density (or electric field strength) at deeper cortical targets. In addition, in the "sham montage," we observed an enhancement of post MEP amplitudes, most probably caused by difficulties of the participants to remain completely relaxed regarding muscle tone over the prolonged time course of the experiment. This most likely also resulted in high inter-individual variability in MEP measures (Figure 5). Another factor which might have contributed to this variability is the substantial intrinsic trialto-trial amplitude variability of MEPs, due to state differences of brain activity, and other factors. The recently introduced EEG-adapted stimulation protocols might be helpful to reduce such variability in future (Zrenner et al., 2018). However, the variability of the MEP difference between the targeted and the contralateral RoIs (i.e., the specificity) was not affected as much (as shown in Figure 6C). Nevertheless, the negative specificity in the "sham montage," in Figure 6C, is notable with the nondominant leg showing higher cortical excitability alterations than the dominant leg. This asymmetry might be related to an impact of foot dominance on MEP, similar to results shown for hand dominance in young adults (Bernard and Seidler, 2012). Since only one montage was tested as sham condition, and post-tDCS measures were covering a shorter time course in the sham as in the real stimulation conditions, blinding might potentially have been compromised in some participants; however, the respective multiple-session experimental design and the randomized order of experiments should have prevented unblinding in most participants.

The results of this study might be relevant for presumptive clinical applications of tDCS for reducing post-stroke maladaptive plasticity at the unaffected contralesional hemisphere that produces inter-hemispheric inhibition (Jones, 2017). While, however, higher specificity of stimulation might be achieved relatively easily in non-lesioned brains via modeling of a standard head, and small electrode sizes might be helpful, this does not easily transfer to patients with brain lesions, in which representations of brain functions, and also physical properties of conductivities, might differ. Here, patient-specific individual head-models may be important to optimize tDCS of the leg motor area to make it a viable clinical option in post-stroke neurorehabilitation (Otal et al., 2016).

\section{CONCLUSION}

We conclude that electrode size, cathodal return electrode position have a relevant impact on anodal tDCS effects on excitability of the lower limb motor cortex. In the "conventiona montage" condition, the large-anode affected both the targeted and the contralateral leg motor representations in a similar way while the small-anode in both the "conventional montage" and the "side montage" primarily affected the targeted leg motor representation in terms of corticospinal excitability alterations. Here the "side montage" resulted in more specific effects. The results of this study show that modeling in combination with 
physiological testing is suited to optimize tDCS protocols, and might be relevant for future studies targeting the lower limb motor cortex.

\section{AUTHOR CONTRIBUTIONS}

$\mathrm{MN}$ and $\mathrm{AD}$ contributed to the conception of this investigation. ÁF substantially contributed to the analysis of the electrophysiological data. ZR substantially contributed to the analysis of the computational data. AD, AF, WP, and MN

\section{REFERENCES}

Alkadhi, H., Crelier, G. R., Boendermaker, S. H., Golay, X., Hepp-Reymond, M.-C., and Kollias, S. S. (2002). Reproducibility of primary motor cortex somatotopy under controlled conditions. Am. J. Neuroradiol. 23, 1524-1532.

Bailey, N. W., Thomson, R. H., Hoy, K. E., Hernandez-Pavon, J. C., and Fitzgerald, P. B. (2016). TDCS increases cortical excitability: direct and Fitzgerald, P. B. (2016). TDCS increases cortical excitability: direct
evidence from TMS-EEG. Cortex 74, 320-322 doi: 10.1016/j-cortex 2014 . evidence

Batsikadze, G., Moliadze, V., Paulus, W., Kuo, M.-F., and Nitsche, M. A. (2013). Partially non-linear stimulation intensity-dependent effects of direct current stimulation on motor cortex excitability in humans. J. Physiol. 591, 1987-2000. doi: $10.1113 /$ jphysiol.2012.249730

Bernard, J. A., and Seidler, R. D. (2012). Hand dominance and age have interactive effects on motor cortical representations. PLoS One 7:e45443. doi: 10.1371/ journal.pone. 0045443

Bikson, M. Datta, A. Rahman, A and Scaturro, J. (2010). Electrode montages for tDCS and weak transcranial electrical stimulation: role of "return" electrode's position and size. Clin. Neurophysiol. 121, 1976-1978. doi: 10.1016/j.clinph. 2010.05 .020

Collins, D. L., Zijdenbos, A. P., Kollokian, V., Sled, J. G., Kabani, N. J., Holmes, C. J., et al. (1998). Design and construction of a realistic digital brain phantom. IEEE Trans. Med. Imaging 17, 463-468. doi: 10.1109/42.71 2135

Das, S., Holland, P., Frens, M. A., and Donchin, O. (2016). Impact of transcranial direct current stimulation (tDCS) on neuronal functions. Front. Nurosci. 10:550. doi: $10.3389 /$ fnins. 2016.00550

Datta, A. Baker J. M Bikson, M., and Fridriksson, J. (2011). Individualized model predicts brain current flow during transcranial direct-current stimulation treatment in responsive stroke patient. Brain Stimul. 4, 169-174. doi: 10.1016/j. brs.2010.11.001

Dutta, A., Chugh, S., Banerjee, A., and Dutta, A. (2014a). Point-of-care-testing of standing posture with Wii balance board and microsoft kinect during transcranial direct current stimulation: a feasibility study. NeuroRehabilitation 34, 789-798. doi: 10.3233/NRE- 141077

Dutta, A., Paulus, W., and Nitsche, M. A. (2012). "Effects of cathode placement on anodal transcranial DC stimulation of leg motor area: a simulation study," in Proceeding of the 2nd IASTED International Conference Assistive Technology in Proceeding of the 2nd IASTED International

Dutta, A., Paulus, W., and Nitsche, M. A. (2014b). Facilitating myoelectric-control with transcranial direct current stimulation: a preliminary study in healthy humans. J. Neuroeng. Rehabil. 11:13. doi: 10.1186/1743-0003-11-13

Fang, Q., and Boas, D. A. (2009). "Tetrahedral mesh generation from volumetric binary and grayscale images," in Proceedings of the IEEE International Symposium on Biomedical Imaging: From Nano to Macro, ISBI'09, Chicago, IL, $1142-1145$

Faria, P., Hallett, M., and Miranda, P. C. (2011). A finite element analysis of the effect of electrode area and inter-electrode distance on the spatial distribution of the current density in tDCS. J. Neural Eng. 8:066017. doi: 10.1088/1741-2560/ of the curr

Fischl, B. (2012). FreeSurfer. Neuroimage 62, 774-781. doi: 10.1016/j.neuroimage. 2012.01.021 contributed to the interpretation of the results and writing and reviewing of the manuscript.

\section{FUNDING}

This work was supported by the (i) Alexander von Humboldt Foundation, Germany; (ii) Campus France (PROCOPE Mobility Grants) and INRIA France; and (iii) German Academic Exchange Service (DAAD). The publication of this article was funded by the Open Access Fund of the Leibniz Association.

Flöel, A. (2014). tDCS-enhanced motor and cognitive function in neurologica diseases. Neuroimage 85, 934-947. doi: 10.1016/j.neuroimage.2013.05.098

Geuzaine, C., and Remacle, J. F. (2009). Gmsh: a 3-D finite element mesh generator with built-in pre-and post-processing facilities. Int. J. Numer. Methods Eng. 79, 1309-1331. doi: 10.1002/nme.2579

Groppa, S., Oliviero, A. Eisen, A., Quartarone, A., Cohen, L., Mall, V., et al. (2012). A practical guide to diagnostic transcranial magnetic stimulation: report of an IFCN committee. Clin. Neurophysiol. 123, 858-882. doi: 10.1016/j.clinph.2012 01.010

Holmes, C. J., Hoge, R., Collins, L., Woods, R., Toga, A. W., and Evans, A. C. (1998) Enhancement of MR images using registration for signal averaging. J. Compu Assist. Tomogr. 22, 324-333. doi: 10.1097/00004728-199803000-00032

Jamil, A., Batsikadze, G., Kuo, H. I., Labruna, L., Hasan, A., Paulus, W., et al. (2017). Systematic evaluation of the impact of stimulation intensity on neuroplastic after-effects induced by transcranial direct current stimulation. $I$. Physiol 595, 1273-1288. doi: 10.1113/[P272738

Jayaram, G, and Stinear, J. W. (2009). The effects of transcranial stimulation on paretic lower limb motor excitability during walking. J. Clin. Neurophysiol. 26 272-279. doi: 10.1097/WNP.0b013e3181af1d41

Jeffery, D. T., Norton, J. A., Roy, F. D., and Gorassini, M. A. (2007) Effects of transcranial direct current stimulation on the excitability of the leg motor cortex. Exp. Brain Res. 182, 281-287. doi: 10.1007/s00221-0071093-y

Jones, T. A. (2017). Motor compensation and its effects on neural reorganization after stroke. Nat. Rev. Neurosci. 18, 267-280. doi: 10.1038/nrn.2017.26

Jurcak, V., Tsuzuki, D., and Dan, I. (2007). 10/20, 10/10, and 10/5 systems revisited: their validity as relative head-surface-based positioning systems. Neuroimage 34, 1600-1611 doi: 10.1016/j neuroimage. 2006.09.024

Kaminski, E., Steele, C. I., Hoff, M., Gundlach, C., Rjosk, V., Sehm, B., et al. (2016) Transcranial direct current stimulation (tDCS) over primary motor cortex les area promotes dynamic balance task performance. Clin. Neurophysiol. 127, 2455-2462. doi: 10.1016/j.clinph.2016.03.018

Koessler, L., Maillard, L., Benhadid, A., Vignal, J. P., Felblinger, J., Vespignani, H. et al. (2009). Automated cortical projection of EEG sensors: anatomical correlation via the international 10-10 system. Neuroimage 46, 64-72. doi: 10.1016/. neuroimage.2009.02.006

Liebetanz, D., Nitsche, M. A., Tergau, F., and Paulus, W. (2002). Pharmacological approach to the mechanisms of transcranial DC-stimulation-induced aftereffects of human motor cortex excitability. Brain 125, 2238-2247. doi: 10.1093/ brain/awf238

Madhavan, S., and Shah, B. (2012). Enhancing motor skill learning with transcranial direct current stimulation-a concise review with applications to stroke. Front. Psychiatry 3:66. doi: 10.3389/fpsyt.2012.00066

Madhavan, S., and Stinear, J. W. (2010). Focal and bidirectional modulation of lower limb motor cortex using anodal transcranial direct current stimulation. Brain Stimul. 3, 42-50. doi: 10.1016/j.brs.2009.06.005

Moliadze, V., Antal, A., and Paulus, W. (2010). Electrode-distance dependent after effects of transcranial direct and random noise stimulation with extracephalic reference electrodes. Clin. Neurophysiol. 121, 2165-2171. doi: 10.1016/..clinph. 2010.04 .033

Monte-Silva, K., Kuo, M.-F., Hessenthaler, S., Fresnoza, S., Liebetanz, D., Paulus, W., et al. (2013). Induction of late LTP-like plasticity in the human 
motor cortex by repeated non-invasive brain stimulation. Brain Stimul. 6 , 424-432. doi: 10.1016/j.brs.2012.04.011

Monte-Silva, K., Kuo, M.-F, Liebetanz, D. Paulus, W, and Nitsche, M. A. (2010).

Shaping the optimal repetition interval for cathodal transcranial direct current stimulation (tDCS). J. Neurophysiol. 103, 1735-1740. doi: 10.1152/jn.00924. 2009

Nitsche, M. A., Fricke, K., Henschke, U., Schlitterlau, A., Liebetanz, D., Lang, N., et al. (2003a). Pharmacological modulation of cortical excitability shifts induced by transcranial direct current stimulation in humans. J. Physiol. 553, 293-301. doi: 10.1113/jphysiol.2003.049916

Nitsche, M. A., Liebetanz, D., Lang, N., Antal, A., Tergau, F., and Paulus, W. (2003b). Safety criteria for transcranial direct current stimulation (tDCS) in humans. Clin. Neurophysiol. 114, 2220-2222. doi: 10.1016/S1388-2457(03) humans.

Nitsche, M., and Paulus, W. (2000). Excitability changes induced in the human motor cortex by weak transcranial direct current stimulation. J. Physiol. 527, 633-639. doi: 10.1111/j.1469-7793.2000.t01-1-00633.x

Nitsche, M. A., and Paulus, W. (2001). Sustained excitability elevations induced by transcranial DC motor cortex stimulation in humans. Neurology 57, 1899-1901. doi: $10.1212 /$ WNL.57.10.1899

Nitsche, M. A., Seeber, A., Frommann, K., Klein, C. C., Rochford, C., Nitsche, M. S., et al. (2005). Modulating parameters of excitability during and after transcranial direct current stimulation of the human motor cortex. J. Physiol. 568, 291-303. direct current stimulation of the

Oostenveld, R., and Praamstra, P. (2001). The five percent electrode system for high-resolution EEG and ERP measurements. Clin. Neurophysiol. 112, 713-719. doi: $10.1016 / S 1388-2457(00) 00527-7$

Opitz, A., Paulus, W., Will, S., Antunes, A., and Thielscher, A. (2015). Determinants of the electric field during transcranial direct current stimulation. Neuroimage 109, 140-150. doi: 10.1016/j.neuroimage.2015.01.033

Otal, B., Dutta, A., Foerster, Á., Ripolles, O., Kuceyeski, A., Miranda, P. C., et al. (2016). Opportunities for guided multichannel non-invasive transcranial current stimulation in poststroke rehabilitation. Front Neurol 7:21. doi: $10.3389 /$ fneur. 2016.00021

Priori, A., Bertolasi, L., Dressler, D., Rothwell, J., Day, B., Thompson, P., et (1993). Transcranial electric and magnetic stimulation of the leg area of the human motor cortex: single motor unit and surface EMG responses in the tibialis anterior muscle. Electroencephalogr. Clin. Neurophysiol. 89, 131-137. doi: 10.1016/0168-5597(93)90095-7

Rawji, V., Ciocca, M., Zacharia, A., Soares, D., Truong, D., Bikson, M., et al. (2018). tDCS changes in motor excitability are specific to orientation of current flow. Brain Stimul. 11, 289-298. doi: 10.1016/j.brs.2017.11.001

Roche, N., Lackmy, A., Achache, V., Bussel, B., and Katz, R. (2011). Effects of anodal transcranial direct current stimulation over the leg motor area on lumbar spinal network excitability in healthy subjects. J. Physiol. 589, 2813-2826, doi: 10.1113/jphysiol.2011.205161

Rossi, S, Hallett, M. Rossini, P. M. Pascual-Leone, A, and Safety of TMS Consensus Group (2009). Safety, ethical considerations, and application guidelines for the use of transcranial magnetic stimulation in clinical practic and research. Clin. Neurophysiol. 120, 2008-2039. doi: 10.1016/j.clinph.2009. 08.016

Rossini, P., Berardelli, A., Deuschl, G., Hallett, M., Maertens de Noordhout, A. Paulus, W., et al. (1999). Applications of magnetic cortical stimulation. Electroencephalogr. Clin. Neurophysiol. Suppl. 52, 171-185.

Sohn, M. K., Jee, S. J., and Kim, Y. W. (2013). Effect of transcranial direct current stimulation on postural stability and lower extremity strength in hemiplegic stroke patients. Ann. Rehabil. Med. 37, 759-765. doi: 10.5535/arm.2013.37 stroke

Tanaka, S., Hanakawa, T., Honda, M., and Watanabe, K. (2009). Enhancement of pinch force in the lower leg by anodal transcranial direct current stimulation. Exp. Brain Res. 196, 459-465. doi: 10.1007/s00221-009-1863-9

Tanaka, S., Takeda, K., Otaka, Y., Kita, K., Osu, R., Honda, M., et al. (2011). Single session of transcranial direct current stimulation transiently increases knee extensor force in patients with hemiparetic stroke. Neurorehabil. Neural Repair 25, 565-569. doi: $10.1177 / 1545968311402091$

van Asseldonk, E. H., and Boonstra, T. A. (2016). Transcranial direct current stimulation of the leg motor cortex enhances coordinated motor output during walking with a large inter-individual variability. Brain Stimul. 9, 182-190. doi: 10.1016/.brs.2015.10.001

Windhoff, M., Opitz, A., and Thielscher, A. (2013). Electric field calculations in brain stimulation based on finite elements: an optimized processing pipelin for the generation and usage of accurate individual head models. Hum. Brain Mapp. 34, 923-935. doi: 10.1002/hbm.21479

Zrenner, C., Desideri, D., Belardinelli, P., and Ziemann, U. (2018). Real-time EEG-defined excitability states determine efficacy of TMS-induced plasticity in Luman motor cortex. Brain Stimul 11, 374-389, doi: 10.1016/jbrs.2017.11.016

Conflict of Interest Statement: The authors declare that the research was conducted in the absence of any commercial or financial relationships that coul be construed as a potential conflict of interest.

Copyright $@ 2018$ Foerster, Rezaee, Paulus, Nitsche and Dutta. This is an open-access article distributed under the terms of the Creative Commons Attribution License (CC BY). The use, distribution or reproduction in other forums is permitted, provided the original author $(s)$ and the copyright owner(s) are credited and that the original publication in this journal is cited, in accordance with accepted academic practice. No use distribution or reproduction is permitted which does not comply with these terms. 


\subsection{Effects of anodal transcranial direct current stimulation over lower limb primary motor cortex on motor learning in healthy individuals}

Studies combining tDCS with motor task performance in healthy individuals (911) and in neurological patients $(12,14,159)$ have shown a performance improvement accomplished by tDCS. The majority of these studies were dedicated to upper limb performance, however few studies have investigated excitabilityenhancing and performance-improving effects of anodal tDCS over the lower limb motor cortex of healthy humans. To investigate the effect of anodal tDCS over the lower limb motor cortex (M1) on lower limb motor learning in healthy volunteers, and to explore the impact of stimulation protocol specifics as well as individual characteristics on stimulation effects, we conducted a randomized, single blind and sham-controlled study. Thirty three (mean age $25.81 \pm 3.85,14$ female) volunteers were included, and received anodal or sham tDCS over the left M1 (M1-tDCS). $0.0625 \mathrm{~mA} / \mathrm{cm}^{2}$ anodal tDCS was applied for 15 minutes during performance of a visuo-motor task (VMT) with the right leg. Motor learning was monitored for performance speed and accuracy based on electromyographic recordings. We also investigated the influence of electrode size and baseline responsivity to transcranial magnetic stimulation (TMS) on the stimulation effects. Relative to baseline measures, only M1-tDCS applied with small electrodes and in volunteers with high baseline sensitivity to TMS significantly improved VMT performance. The computational analysis showed that the small anode was more specific to the targeted leg motor cortex volume when compared to the large anode. We conclude that anodal M1tDCS modulates VMT performance in healthy subjects. Since these effects critically depend on sensitivity to TMS and electrode size, future studies should investigate the effects of intensified tDCS and/or model-based different electrode positions in lowsensitivity TMS individuals. 


\title{
Effects of anodal transcranial direct current stimulation over lower limb primary motor cortex on motor learning in healthy individuals
}

\author{
Águida Foerster, ${ }^{1,2}$ (D) Anirban Dutta, ${ }^{3}$ Min-Fang Kuo, ${ }^{2}$ Walter Paulus ${ }^{1}$ and Michael A. Nitsche $e^{2,4}$ \\ ${ }^{1}$ Department of Clinical Neurophysiology, Universitätsmedizin Göttingen, Georg-August Universität, Göttingen, Germany \\ ${ }^{2}$ Deptartment Psychology and Neurosciences, Leibniz Research Centre for Working Environment and Human Factors, \\ Leibniz-Institut für Arbeitsforschung, Ardeystr. 67, 44139 Dortmund, Germany \\ ${ }^{3}$ Department of Biomedical Engineering. University at Buffalo, Buffalo, NY, USA \\ ${ }^{4}$ Department of Neurology, University Medical Hospital Bergmannsheil, Bochum, Germany
}

Keywords: biofeedback, motor task, neuromodulation, transcranial direct current stimulation

Abstract

Transcranial direct current stimulation (tDCS) is a neuromodulatory technique which alters motor functions in healthy humans and in neurological patients. Most studies so far investigated the effects of tDCS on mechanisms underlying improvements in upper limb performance. To investigate the effect of anodal tDCS over the lower limb motor cortex (M1) on lower limb motor learning in healthy volunteers, we conducted a randomized, single-blind and sham-controlled study. Thirty-three $(25.81 \pm 3.85,14$ female) volunteers were included, and received anodal or sham tDCS over the left M1 (M1-tDCS); $0.0625 \mathrm{~mA} / \mathrm{cm}^{2}$ anodal tDCS was applied for 15 min during performance of a visuo-motor task (VMT) with the right leg. Motor learning was monitored for performance speed and accuracy based on electromyographic recordings. We also investigated the influence of electrode size and baseline responsivity to transcranial magnetic stimulation (TMS) on the stimulation effects. Relative to baseline measures, only M1-tDCS applied with small electrodes and in volunteers with high baseline sensitivity to TMS significantly improved VMT performance. The computational analysis showed that the small anode was more specific to the targeted leg motor cortex volume when compared to the large anode. We conclude that anodal M1-tDCS modulates VMT performance in healthy subjects. As these effects critically depend on sensitivity to TMS and electrode size, future studies should investigate the effects of intensified tDCS and/or model-based different electrode positions in low-sensitivity TMS individuals.

\section{Introduction}

Transcranial direct current stimulation (tDCS) is a non-invasive brain stimulation tool used in clinical and experimental settings to induce changes in cortical excitability and modulate cognitive and motor processes (Hummel \& Cohen, 2006; Ziemann et al., 2008; Madhavan \& Shah, 2012). Cortical activity and excitability ar altered by tDCS (Jeffery et al., 2007: Soekadar et al., 2014; Marquez et al., 2015) via immediate changes in neuronal firing by hyperpolarizing or depolarizing brain tissue, and glutamatergic NMDA receptor-dependent plasticity (Liebetanz et al., 2002; Stagg \& Nitsche, 2011). In recent years, the potential to combine tDC with rehabilitation to improve motor recovery of neurological

Correspondence: Michael Nitsche, ${ }^{2}$ Deptartment Psychology and Neurosciences, as above. E-mail: nitsche@ifado.de

Received 26 October 2017, revised 7 February 2018, accepted 7 February 2018

Edited by Gregor Thut. Reviewed by Sangeetha Madhavan. University of Illinois at Chicago, USA; and Michael Ridding, The University of Adelaide, Australi patients by modulating synaptic efficacy with tDCS emerged (Kumru et al., 2016). Respective recovery processes are intrinsically linked to shifts in cortical excitability, which may share mechanisms with tDCS-induced neuromodulation (Campanac \& Debanne, 2007; Bolognini et al., 2009). Corroborating this theory, studies combining tDCS with motor task performance in healthy individuals (Foerste et al. 2013. Cabral et al., 2015. Hashemirad et al. 2016) and in neurological patients (Nair et al., 2011; Kang et al., 2016; Rocha et al., 2016) improved performance. However, the majority of these studies were dedicated to upper limb performance, the effects of tDCS on lower limb function are relatively understudied (Fleming et al., 2017), and stimulation parameters and experimental designs are heterogeneous (see Table 1).

One of the initial studies on tDCS effects on leg motor cortex excitability in healthy humans showed that anodal tDCS $\left(35 \mathrm{~cm}^{2}\right.$ electrode size, $2 \mathrm{~mA}$ stimulation intensity, 10-min stimulation duration) over the leg area of the motor cortex increased corticospina excitability of the tibialis anterior (TA) muscle representation, reflected by an increase in the amplitude of motor evoked potentials (MEP). MEP amplitudes recorded at rest and during active 
2 A. Foerster et al.

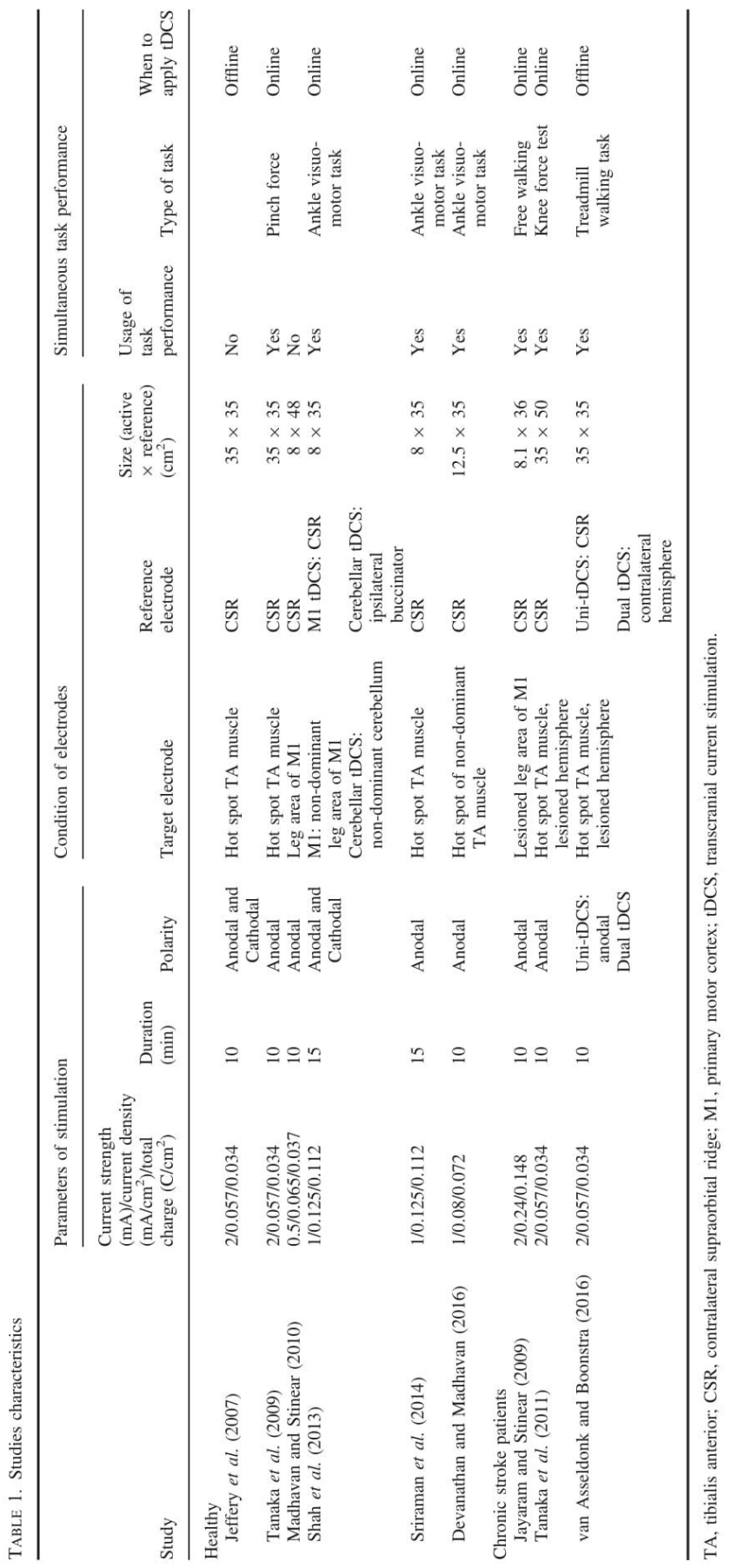

C 2018 Federation of European Neuroscience Societies and John Wiley \& Sons Ltd
European Journal of Neuroscience, 1-11 
contraction were increased following anodal tDCS by 59 and $35 \%$, and remained elevated for up to $60 \mathrm{~min}$ after intervention (Jeffery et al., 2007). However, cathodal tDCS did not suppress leg motor cortex excitability in difference to the impact of cathodal tDCS on the hand motor area, which may be related to differences in orientation and position of the leg motor cortex, or fewer inhibitory circuits available in this area (Porter \& Lemon, 1993; Hallett, 2003; Laczó et al., 2014). Similar effects were described in another study for anodal tDCS $\left(8 \mathrm{~cm}^{2}\right.$ electrode size, $0.5 \mathrm{~mA}$ stimulation intensity, 10-min stimulation duration) of lower limb representations; her interestingly, stimulation of the target region resulted in antagonistic excitability alterations of the contralateral homologue (Madhavan \& Stinear, 2010). For remote effects, it has been shown that anoda tDCS $\left(35 \mathrm{~cm}^{2}\right.$ electrode size, $2 \mathrm{~mA}$ stimulation intensity, 20-min stimulation duration) over the lower limb motor cortex induced effects on spinal network excitability similar to those observed during co-contraction (Roche et al., 2011). For effects of lower limb tDCS on motor performance in healthy humans, Tanaka and colleagues (Tanaka et al., 2009) showed that anodal tDCS $\left(35 \mathrm{~cm}^{2}\right.$ electrode size, $2 \mathrm{~mA}$ stimulation intensity, 10-min stimulation duration) transiently enhanced maximal leg pinch force for up to $30 \mathrm{~min}$ after stimulation compared to baseline. In agreement with the abovementioned physiological study (Jeffery et al., 2007), cathodal tDCS did not change performance. Improvement in target-tracking accuracy of the ankle was observed after anodal tDCS $\left(8 \mathrm{~cm}^{2}\right.$ electrode size, $1 \mathrm{~mA}, 15$-min stimulation duration) over the non-dominant lower limb motor cortex or cerebellum applied during task performance, and again, no effects of M1 lower limb cathodal tDCS wer observed (Shah et al., 2013). Another study showed that the effect of anodal tDCS $\left(8 \mathrm{~cm}^{2}\right.$ electrode size, $1 \mathrm{~mA}$ stimulation intensity, 15-min stimulation duration) on ankle motor skill learning are timing-dependent, with better results when the stimulation is applie during task performance (Sriraman et al., 2014). Finally, Devanathan and Madhavan (Devanathan \& Madhavan, 2016) observed that anodal tDCS $\left(12.5 \mathrm{~cm}^{2}\right.$ electrode size, $1 \mathrm{~mA}$ stimulation intensity, 10-min stimulation duration) over the non-dominant lower limb motor cortex improves reaction time of ankle task performance. Thus taken together, evidence for excitability-enhancing and performance-improving effects of anodal tDCS over the lower limb moto cortex of healthy humans is available.

With regard to patient studies, Jayaram and Stinear (Jayaram \& Stinear, 2009) studied the neuromodulatory effects of anodal tDCS $\left(8.1 \mathrm{~cm}^{2}\right.$ electrode size, $2 \mathrm{~mA}$ stimulation intensity, 10-min stimulation duration) over the ipsilesional motor cortex in chronic stroke patients. Stimulation increased paretic limb and decreased non-paretic limb motor excitability. These relatively focal effects might be explained by the high current density $\left(0.24 \mathrm{~mA} / \mathrm{cm}^{2}\right)$ applied ove the lower limb motor cortex with small electrodes. Positve tDCS effect was observed as well when a single session of anodal tDC ( $35 \mathrm{~cm}^{2}$ electrode size, $2 \mathrm{~mA}$ stimulation intensity, 10-min stimulation duration) over the paretic lower limb motor cortex representation increased knee extensor force in patients with hemiparetic stroke for up to $30 \mathrm{~min}$ following intervention (Tanaka et al. 2011). It was thus postulated that tDCS, when combined with lower extremity strength training, may facilitate post-stroke rehabilitation. Also, a recent study showed beneficial effects of anodal tDCS ( $35 \mathrm{~cm}^{2}$ electrode size, $2 \mathrm{~mA}$ stimulation intensity, 10-min stimulation duration) over the lesioned hemisphere on coordinated moto output during walking with however large interindividual variability (van Asseldonk \& Boonstra, 2016)

Considering the depth of the anatomical representation of the lower limb motor cortex, the relatively high specificity of effect obtained in previous studies with small electrodes and the functional relevance of the TA muscle for walking, in this study we aimed to evaluate the effects of high current density applied through a relatively small $\left(8 \mathrm{~cm}^{2}\right)$ stimulation electrode over the TA muscle motor cortex representation on motor performance in healthy individuals. We specifically aimed to investigate tDCS-induced facilitation of myoelectric control in terms of response time and accuracy during performance of a visuo-motor task (VMT) that was used in our prior work with larger $5 \times 7 \mathrm{~cm}$ stimulation electrodes in healthy individuals (Dutta et al., 2014). Based on the relevance of stimulation focality, which is particularly challenging for cortical areas remote from the brain surface as the leg, we also investigated the specificity of tDCS by finite element modeling regarding two different sizes of electrodes $\left(8 \mathrm{~cm}^{2}\right.$ vs. $\left.35 \mathrm{~cm}^{2}\right)$. As tDCS had interindividual heterogeneous effects on motor performance, and sensitivity to transcranial magnetic stimulation (TMS) has been revealed as a potential marker of responsivity to tDCS for the upper limb motor cortex (Jamil et al., 2017), we furthermore aimed to explore the relevance of this parameter for the stimulation effects.

\section{Methods}

Subjects

Thirty-three (19 men, 14 women, $25.81 \pm 3.85$ years old) healthy right-handed individuals consented to participate in this singleblinded study, which was performed in accordance with the Declaration of Helsinki and approved by the Ethics Committee of the University Medical Center of the University of Goettingen, Germany. All participants were financially compensated. None of them was taking acute or regular CNS-active medication, had a history of neurological or psychological disorders, any contraindications to tDCS, or were smokers. The volunteers were instructed not to practice sport activities or consume alcohol during $24 \mathrm{~h}$ before the experimental session, and the last caffeine consumption had to be at least $2 \mathrm{~h}$ before the start of the experiment. The volunteers were randomly divided into two groups - anodal tDCS $(n=18)$ and sham tDCS $(n=15)$.

\section{Experimental procedures}

Participants were comfortably seated in a chair centered at roughly $60 \mathrm{~cm}$ distance from a computer screen. They performed a visuomotor task (VMT) before, during and after application of anodal or sham tDCS. The VMT was conducted with biofeedback provided on the computer screen (screen size $=31.5 \times 19 \mathrm{~cm}$ and resolution $=1280 \times 1024)$ that required the subject to volitionally activate the TA muscle of the right leg (Fig. 1). Before starting the VMT, the maximum voluntary contraction (MVC) of the subject was measured and entered in custom-written VMT software running in MATLAB (The MathWorks Inc., USA). For the MVC measurement, isometric contraction of the target muscle against a resistance was kept constant for $3 \mathrm{~s}$, and the average rectified EMG during those seconds was used as the MVC value.

\section{Visuo-motor task}

During the VMT, the custom-made software presented visual cues on the computer screen. The participants had to contract the TA muscle isometrically against a resistance as accurate as possible (no instruction about the importance of performance speed was given) in response to the visual cue-TARGET. The TARGET was presented 
4 A. Foerster $e$ al.
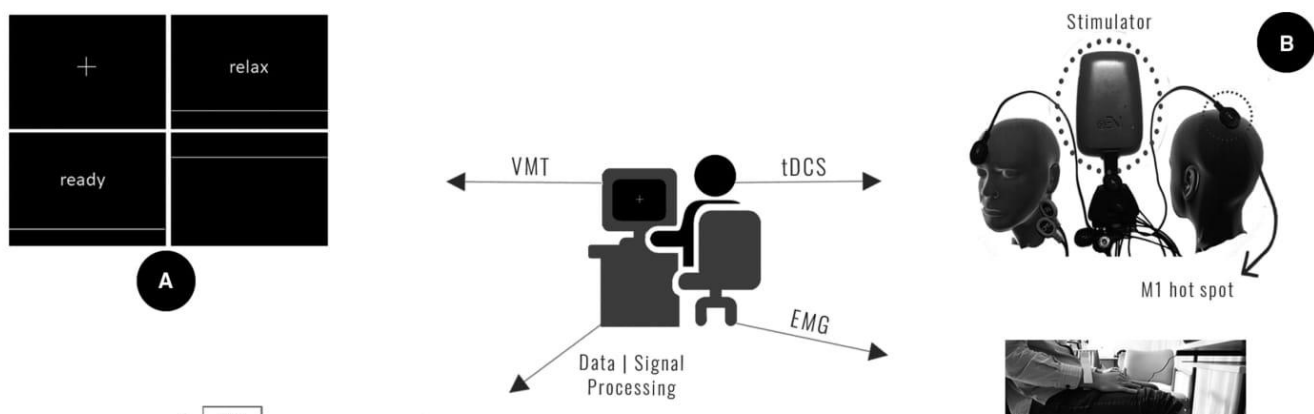

M1 hot spot

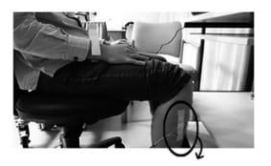

Tibialis anterior muscle
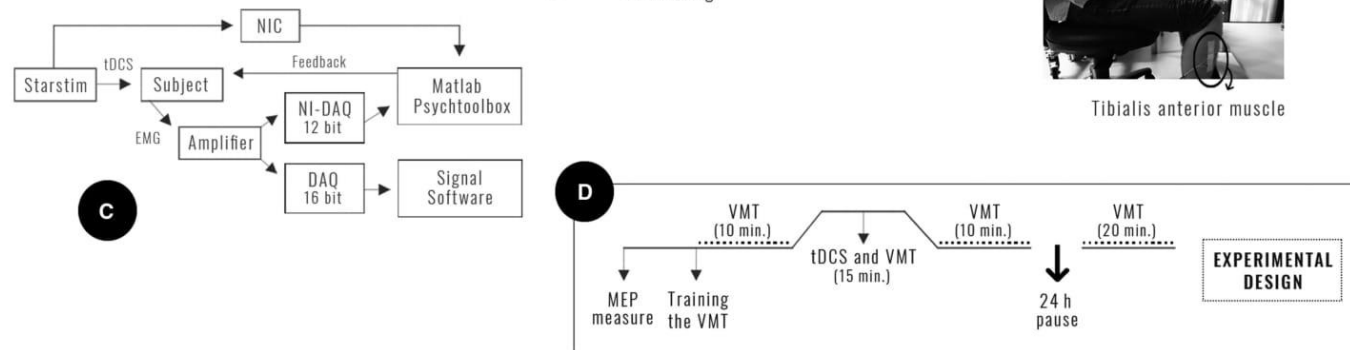

FIG. 1. Experimental setup and design. Participants were asked to sit in a comfortable chair centered at roughly $60 \mathrm{~cm}$ distance from a computer screen. (A) Visuo-motor task: the software presented visual cues (relax, ready and the target, which was a horizontal line on the screen presented at a position which repreVisuo-motor task: the software presented visual cues (relax, ready and the target, which was a horizontal line on the screen presented at a position which represented a degree of muscle contraction between 40 and $80 \%$ of individual maximal voluntary contraction), and the subjects had to follow the target position on the screen anterior tibial muscle. (B) For transcranial direct current stimulation (IDS) was placed above Deforinteers were submitted to two experimental sessions.

as a horizontal line across the full computer screen that jumped to a randomized value between 40 and $80 \%$ of the subject's MVC Visual feedback of muscle activity was provided with a moving average of the rectified EMG in a sliding window of $500 \mathrm{~ms}$ that was normalized to MVC, which was represented by a second horizontal line-CURSOR - with identical proportional dynamics as the TARGET signal. The TARGET cue was presented for $5 \mathrm{~s}$. The participants had to isometrically contract the TA muscle to match the CURSOR to the TARGET line on the computer screen. Each TARGET cue was preceded by a RELAX (duration $=10 \mathrm{~s}$ ) and a READY cue (duration randomized between 3 and $5 \mathrm{~s}$ to prevent anticipatory responses) during each VMT trial (Fig. 1A). In total, 75 trials ( 15 pre, 45 during and 15 post) were performed. Before each block of VMT, we installed a 3-min resting period to avoid muscle fatigue.

\section{tDCS protocol}

Transcranial direct current stimulation was applied through salinesoaked surface sponge electrodes $\left(8 \mathrm{~cm}^{2}\right.$ for the target electrode and $88 \mathrm{~cm}^{2}$ for the return electrode). The anode target electrode was centered over the hot spot of the left M1 cortical representation of the right leg TA muscle (discussed in Section Transcranial magnetic current stimulation). The cathode return electrode was positioned above the contralateral supraorbital ridge (Fig. 1B). We have chosen this relatively large return electrode size $\left(88 \mathrm{~cm}^{2}\right)$ to reduce current density under this electrode and prevent an impact of the return electrode on excitability of the cortex under that electrode (Nitsche et al., 2007). tDCS was applied with a current intensity of $0.5 \mathrm{~mA}$ for $15 \mathrm{~min}$ during VMT performance, with a 10 -s ramp up at the beginning and a 10-s ramp down at the end of stimulation. We stimulated with a current density of $0.0625 \mathrm{~mA}$ $\mathrm{cm}^{2}$ and delivered a total charge density of $0.056 \mathrm{C} / \mathrm{cm}^{2}$. In the sham condition, the electrode setup was the same, but the current was turned off after $30 \mathrm{~s}$. tDCS was applied by a Starstim stimulator (NeuroElectrics, Spain) using Neuroelectrics Instrument Controller (NIC) v1.2 software (Fig. 1C). NIC uses the clock from the Starstim as master for timing, which was received by NIC through wireless data streaming.

Subjective side effects were collected by a questionnaire (Brunoni et al., 2011) including headache, neck pain, scalp pain, tingling, itching, burning, skin redness, sleepiness, trouble of concentrating and acute mood changes immediately after delivery of stimulation and VMT performance.

\section{Transcranial magnetic current stimulation}

In the real stimulation group, TMS was used to identify the best motor cortical localization (hot spot) of the right TA muscle. The hot spot was determined via motor evoked potential recordings (MEP), elicited by a Magstim 200 stimulator (Magstim, Dyfed, UK) with a figure-of-eight coil (diameter $70 \mathrm{~mm}$ ). The hot spot corresponded to the coil position which elicited the largest MEP with a given stimulation intensity (Rossini et al., 1994), resulting in MEP average of at least $0.5 \mathrm{mV}$ peak-to-peak after 20 of successive TMS pulses (Madhavan et al., 2011). 
According to the results of a previous experiment, which had shown for upper limb effects of tDCS that sensitivity to TMS is a relevant factor for efficacy of the intervention (Labruna et al. 2016), we also investigated whether baseline motor cortex excitability affected the tDCS effects. Therefore, we recruited volunteers in which MEPs could be elicited in the resting muscle condition (resting MEP group, $n=9$ ) with a percentage of maximum stimulator output (\% MSO) of smaller than $90 \%$, and volunteers in which clearly identifiable MEPs could only be obtained under slight contraction (about $0.2 \mathrm{mV}$ contraction, monitored online through electromyography). The ability to induce MEP during rest or only during moderate muscle contraction was the criterion to split the participants in high vs. low TMS sensitivity subgroups, and we performed an additional analysis of the data from the main experiment for these high and low TMS sensitivity groups in the rea tDCS condition.

\section{Experimental sessions}

Figure 1D explains the experimental design of this study. After receiving informed consent from the participants, the motor hot spot of the right leg TA muscle was identified. After this measure and positioning of the tDCS electrodes over the motor hot spot, participants underwent a practice session of the VMT (15 trials) to get acquainted to the procedure. After this practice session, baseline VMT ( 15 trials) was performed for $10 \mathrm{~min}$ followed by $15 \mathrm{~min}$ of VMT (45 trials) combined with tDCS, and then post-tDCS (15 trials) for another $10 \mathrm{~min}$. To evaluate the long-term effects of tDCS on performance, the volunteers performed 20-min VMT (60 trials) again after $24 \mathrm{~h}$ (next day).

\section{Secondary experiment}

Based on the insights gained from finite element modeling (discussed in the following section), we performed a separate exploratory experiment with six subjects of the anodal tDCS group, to further explore whether the size of the target electrode affected the tDCS effects, because in different previous experiments different electrode sizes were used. After participation in the main experiment, these subjects took part in this secondary experiment. Experi mental procedures were identical to those of main experiment. Here, we used a $35 \mathrm{~cm}^{2}$ target electrode and $2 \mathrm{~mA}$ current intensity. These stimulation parameters resulted in $0.057 \mathrm{~mA} / \mathrm{cm}^{2}$ current density, a similar value as that obtained with the smaller (area: $8 \mathrm{~cm}^{2}$ ) target electrode.

\section{Finite element modeling}

We leveraged the simnIBs software pipeline to develop the head model for finite element modeling (Windhoff et al., 2013). SIMNIBS incorporates FreeSurfer tools (Fischl, 2012) to segment the brain and FSL (Jenkinson et al., 2012) BET/BETsurf tools to segment the rest of the head. This software pipeline was applied on the Colin27 average brain, which is based on 27 MRI scans conducted in one individual, and linear registration of the images to create an average with high signal-to-noise ratio and structure definition (Holmes et al., 1998). Tetrahedral head meshes from the Colin27 average brain MRI data were generated using the 'mri2mesh' tool in the SIMNIBS software pipeline (Windhoff et al., 2013) for the MRI-based head model with an average tetrahedron volume of $1 \mathrm{~mm}^{3}$. Electrode positions were defined by the International 10-20 system with fiducials at $\mathrm{Nz}, \mathrm{Iz}$, right and left preauricular points for registration with the head model (Jurcak et al., 2007). The target electrode for anodal tDCS (run 1: area $8 \mathrm{~cm}^{2}$ with $0.5 \mathrm{~mA}$, run 2: area $35 \mathrm{~cm}^{2}$ with $2 \mathrm{~mA}$ ) was placed $15 \mathrm{~mm}$ lateral and $20 \mathrm{~mm}$ posterior to $\mathrm{Cz}$ (approximate TA muscle hot spot derived from experimental data; Dutta et al., 2014), and the cathode $\left(\right.$ area $88 \mathrm{~cm}^{2}$ ) placed at Fp2 (above the contralateral supraorbital ridge) according to the 10-10 EEG system (Oostenveld \& Praamstra, 2001). The electric field values at the nodes within the bilateral leg area cluster were captured with a sphere with a radius of $1 \mathrm{~cm}$ centered at the geometric centers of gravity (COG) $(-7,-38,75 \mathrm{~mm})$ and $(6,-38,75 \mathrm{~mm})$ in MNI coordinates, based on prior works (Alkadhi et al., 2002; Otal et al., 2016). All node values within the clusters were imported in MAтLAв (The MathWorks Inc.) to compute average electric field $(\overrightarrow{E F})$ magnitude and direction. The specificity of the electric field at the targeted ( $\operatorname{targ})$ and contralateral (contra) leg motor volume ( -7 , $-38,75 \mathrm{~mm})$ and $(6,-38,75 \mathrm{~mm})$ in MNI coordinates, respectively, for run 1 and 2 was computed based on the average magnitude $(|E F|=\sqrt{\overrightarrow{E F} \cdot \overrightarrow{E F}})$

$$
\text { Specificity }=\frac{\left(|E F|_{\text {targ }}-|E F|_{\text {contra }}\right)}{\left(|E F|_{\text {targ }}+|E F|_{\text {contra }}\right)}
$$

The average electric field $(\overrightarrow{E F})$ unit vector was computed at the targeted (targ) and contralateral (contra) leg motor volume for comparison.

\section{EMG signal analysis}

EMG was collected from the TA muscle according to SENIAM guidelines (http://www.seniam.org/), amplified and band-pass-filtered (frequency band $=10-500 \mathrm{~Hz}$ ) before being sampled at $2000 \mathrm{~Hz}$ by a 12-bit data acquisition (DAQ) system (National Instruments, USA). Psychtoolbox (http://psychtoolbox.org/) was used to develop the graphical user interface for the visuo-motor task. EMG was also recorded separately in the computer using SIGNAL software (http:// www.ced.co.uk/pru.shtml?sig3wglu.htm) via a 16-bit DAQ for offline analysis. The Cursor Time was determined offline as the time period spanning from the instant of visual TARGET presentation until the CURSOR matched the TARGET during VMT. Performance speed was determined offline as the time period spanning from the instant of the visual TARGET presentation until the rectified EMG deviated more than three times from the standard deviation of the resting value (before appearance of the TARGET cue) Accuracy was determined in terms of root mean square error (RMSE) between the CURSOR and the TARGET signals during the $5 \mathrm{~s}$ of TARGET cue presentation. A custom MATLAB script was written for these computations.

\section{Statistical analysis}

We performed a descriptive analysis for group characteristics. Comparisons between groups, according to the demographic characteristics of the samples, were performed using independent-samples Student's $t$-test for continuous and the chi-squared test for categorical factors. Side effects immediately after M1 tDCS and sham were analyzed by Fisher's exact test.

Before statistical analysis, trials with values deviating more than \pm 2 standardized deviations from the mean were excluded. Only results of volunteers with minimum five trials remaining in each block were included in the statistical analyses. After exclusion of trials which deviated more than two standard deviations from 
individual mean performance level, for performance speed and accuracy, about $90 \%$ of the trials remained in both experimental groups, and for cursor time, $90 \%$ of the trials remained in the real stimulation group and $70 \%$ of the trials remained in the sham group. EMG data (performance speed, cursor time and accuracy) showed a normal distribution according to the results of respective KolmogorovSmirnov tests; thus, we performed parametric tests in our statistical analysis. A two-tailed independent-samples $t$-test was conducted to evaluate whether the baseline measures differed between the tDCS experimental groups for the main experiment, a two-tailed pairedsamples $t$-test for the results of the secondary experiment and a oneWay ANOVA for the re-analyzed data from the main experiment, which included baseline TMS sensitivity as additional factor. Mixed model two-way ANOVAS with the between-subject factor tDCS group (real vs. sham), the repeated measure factor time (pre, during, post and $24 \mathrm{~h}$ after intervention) and the dependent variables, accuracy, cursor time and performance speed, were performed for the results of the main experiment (see Section Experimental procedures for description), including anodal tDCS group vs. sham group, as well as active MEP tDCS vs. resting MEP tDCS group vs. sham (re-analyzed data), which included a distinction of the anodal stimulation group according to baseline motor cortex excitability. Regarding the secondary experiment concerning electrode size (see Section Secondary experiment for description), a repeated-measures ANOVA with the repeated measure factors electrode size $(8$ anc $35 \mathrm{~cm}^{2}$ ) and time was performed for each outcome parameter. We conducted the Mauchly sphericity test to assess the validity of the sphericity assumption, and the Greenhouse-Geisser correction was applied when necessary. Conditional on significant results of the mixed model two-way and repeated-measures ANOVAs, we performed post hoc tests by two-tailed $t$-tests for the main and secondary experiments. All analyses were performed with absolute values, an the data were analyzed with spss (Statistical Package for Social Sciences) version 24.0 for Windows (SPSS Inc., Chicago, IL, USA). A $P$ value of $\leq 0.05$ was considered significant for all statistical analyses.

\section{Results}

Results presented in Table 2 show no demographic differences between groups, indicating that the randomization procedure was successful. No difference was observed between groups regarding side effects, and most of the volunteers in either group mentioned no or small (i.e., tingling and itching) adverse effects during or after the tDCS sessions, as shown in Table 3 . The main side effect was sleepiness. The baseline measures for performance speed, cursor time and accuracy did not differ significantly between the experimental groups in the main and in the secondary experiments.

The mixed model two-way ANOvAs revealed a significant effect of tDCS group and time for accuracy, and of time for cursor time. No significant effects were observed for performance speed, and no

TABLE 2. Participants' demographic characteristics

\begin{tabular}{lccc}
\hline & M1 tDCS & Sham tDCS & $P$ value \\
\hline $\begin{array}{l}\text { Gender }-n(\%) \\
\text { Male }\end{array}$ & $13(72.2)$ & $6(40)$ & \\
Female & $5(27.8)$ & $9(60)$ & $0.39^{*}$ \\
Age & $26.9(21-37)^{\dagger}$ & $24.5(22-30)$ & $0.08^{\ddagger}$ \\
\hline
\end{tabular}

${ }^{*}$ Chi-squared test. ${ }^{\dagger}$ Mean age range. ${ }^{\star}$ Independent-samples $t$-test; $P<0.05$. interaction between tDCS group and time was present in the main experiment (Table 4). Relative to baseline, post hoc analysis for cursor time showed a significant $11 \%$ decrease $(P=0.020) 24$-h postintervention (post_24h) only when anodal tDCS was applied (Fig. 2A). Regarding accuracy of contraction, we noted a significant decrease in RMSE values (indicating increased accuracy) during tDCS (stm) and immediately after tDCS (post) compared to baseline for both, the M1 anodal tDCS $\left(7.2 \%\right.$ decrease, $P_{\text {stm }}=0.009$ and $\left.P_{\text {post }}=0.018\right)$ and sham $\left(7 \%\right.$ decrease, $P_{\text {stm }}=0.006$ and $7.7 \%$ decrease, $\left.P_{\text {post }}=0.004\right)$ groups. However, only the M1 anodal tDCS group showed a significant decrease $(45 \%$ decrease, $P=0.001) 24$ $\mathrm{h}$ post-intervention relative to baseline. Furthermore, relative to $24-\mathrm{h}$ post-intervention only for the M1 anodal tDCS group, a significant difference was observed in relation to the during stimulation $(P=0.001)$ and immediately post-stimulation conditions $(P=0.001)$ (Fig. 3A).

For the re-analyzed data (resting MEP $\times$ active MEP $\times$ sham group), which discerned between high and low TMS sensitivity groups in the real tDCS condition, the mixed model ANOvas revealed a significant effect of time and a significant interaction between time and group for accuracy, and of time for cursor time. No significant effects were observed for performance speed (Table 4). The exploratory post hoc analysis revealed that cursor time decreased significantly 24-h post-intervention relative to baseline only in the participants in which MEP were obtained in the resting TA muscle (21.4\% decrease, $P=0.004$, Fig. 2 B). For the accuracy improvement, the post hoc analysis between groups showed significant differences between the resting MEP group and sham during $(P=0.025)$ and immediately after $(P=0.03)$ stimulation, suggesting enhanced accuracy in the sham as compared to the respective real tDCS group. No differences were observed between the active MEP group on the one hand and sham, and resting MEP groups on the other hand. For the factor time, the post hoc tests revealed significant differences between baseline and during stimulation conditions for the active MEP (4.9\% decrease, $P=0.043)$ and sham groups ( $7 \%$ decrease, $P=0.006$ ), and between baseline and immediately after stimulation for the resting MEP $(9.5 \%$ decrease, $P=0.05)$ and sham group $(7.7 \%$ decrease, $P=0.004)$. However, only for the resting MEP group a significant accuracy improvement after $24 \mathrm{~h}$ of stimulation in relation to baseline performance $53 \%$ decrease, $P=0.008)$, during $(48.6 \%$ decrease, $P=0.007)$ and immediately after $(48.2 \%$ decrease, $P=0.011)$ stimulation was observed (Fig. 3B)

In the secondary experiment, in which we compared the effect of different electrode sizes (see Section Secondary experiment for description), the repeated-measures ANOVAs revealed a significant effect of time for accuracy. No significant effects were observed for cursor time and performance speed, and no significant main effect of tDCS group or interaction between tDCS group and time was revealed (Table 5). The exploratory post hoc tests showed a significant decrease in RMSE (i.e., an improvement in accuracy) for all time points $(7.25 \%$ decrease, stm: $P=0.019$; post: $7.25 \%$ decrease, $P=0.032$; post_24h: $52 \%$ decrease, $P=0.027$ ) relative to baseline only when stimulation was applied with small electrodes. Furthermore, a significant accuracy improvement between 24 -h post-intervention and during $(P=0.029)$, and immediately after stimulation $(P=0.030)$ was observed only when the stimulation was applied with small electrodes (Fig. 3C).

Table 6 shows the electric field magnitude from the computational analysis of the head model for simulation run $1\left(8 \mathrm{~cm}^{2}\right.$, $0.5 \mathrm{~mA})$ and run $2\left(35 \mathrm{~cm}^{2}, 2 \mathrm{~mA}\right)$. The electric field vector at the targeted (targ) leg motor volume - sphere with a radius of $1 \mathrm{~cm}$ 
TABLE 3. Side effects immediately after tDCS

\begin{tabular}{|c|c|c|c|c|c|c|c|c|c|c|c|}
\hline Score & Headache & Neck pain & Scalp pain & Tingling & Itching & Burning & Skin redness & Sleepness & Trouble concentrating & Acute mood change & Other \\
\hline \multicolumn{12}{|c|}{ M1 tDCS } \\
\hline 1 & 14 & 17 & 16 & 12 & 11 & 15 & 5 & 2 & 9 & 17 & 18 \\
\hline 2 & 3 & 1 & 1 & 4 & 5 & 2 & 10 & 6 & 7 & 1 & 0 \\
\hline 3 & 1 & 0 & 0 & 2 & 1 & 1 & 3 & 9 & 2 & 0 & 0 \\
\hline 4 & 0 & 0 & 1 & 0 & 1 & 0 & 0 & 1 & 0 & 0 & 0 \\
\hline \multicolumn{12}{|c|}{ Sham tDCS } \\
\hline 1 & 15 & 12 & 13 & 10 & 13 & 12 & 9 & 3 & 5 & 13 & 15 \\
\hline 2 & 0 & 2 & 2 & 5 & 2 & 2 & 6 & 5 & 10 & 2 & 0 \\
\hline 3 & 0 & 1 & 0 & 0 & 0 & 1 & 0 & 6 & 0 & 1 & 0 \\
\hline 4 & 0 & 0 & 0 & 0 & 0 & 0 & 0 & 1 & 0 & 0 & 0 \\
\hline$P^{*}$ & 0.07 & 0.314 & 0.414 & 0.247 & 0.262 & 0.970 & 0.077 & 0.891 & 0.123 & 0.314 & 1.0 \\
\hline
\end{tabular}

A score of 4 corresponds to a severe side effect and 1 means absent side effect. M1: lower limb primary motor cortex. *P values of Fisher's exact test (level of significance $P \leq 0.05)$.

TABLE 4. Results of the mixed model ANOVAS performed for the main experiment and the re-analyzed data of the main experiment introducing baseline motor cortex excitability for each outcome measure

\begin{tabular}{|c|c|c|c|c|}
\hline & df & $F$ & $P$ & $p \eta^{2}$ \\
\hline \multicolumn{5}{|l|}{ Main experiment } \\
\hline \multicolumn{5}{|l|}{ Performance speed } \\
\hline Time* & 1 & 1.323 & 0.260 & 0.045 \\
\hline Group ${ }^{\dagger}$ & 1 & 0.876 & 0.357 & 0.03 \\
\hline Time $\times$ group & 1 & 0.439 & 0.513 & 0.015 \\
\hline \multicolumn{5}{|l|}{ Cursor time } \\
\hline Time & 1 & 9.712 & 0.004 & 0.265 \\
\hline Group & 1 & 0.714 & 0.406 & 0.026 \\
\hline Time $\times$ group & 1 & 0.233 & 0.633 & 0.009 \\
\hline \multicolumn{5}{|l|}{ Accuracy } \\
\hline Time & 1 & 7.763 & 0.009 & .217 \\
\hline Group & 1 & 4.451 & 0.044 & 0.137 \\
\hline Time $\times$ group & 1 & 2.088 & 0.16 & 0.069 \\
\hline \multicolumn{5}{|c|}{$\begin{array}{l}\text { Re-analyzed data from the main experiment including baseline motor cortex } \\
\text { excitability }\end{array}$} \\
\hline \multicolumn{5}{|c|}{ Performance speed } \\
\hline Time & 1 & .013 & 0.91 & 0.001 \\
\hline Group & 2 & 1.42 & 0.259 & 0.095 \\
\hline Time $\times$ group & 2 & 1.15 & 0.322 & 0.078 \\
\hline \multicolumn{5}{|l|}{ Cursor time } \\
\hline Time & 1 & 10.08 & 0.04 & 0.279 \\
\hline Group & 2 & 0.649 & 0.531 & 0.048 \\
\hline Time $\times$ group & 2 & 0.967 & 0.394 & 0.069 \\
\hline \multicolumn{5}{|l|}{ Accuracy } \\
\hline Time & 1 & 19.501 & 0.001 & 0.419 \\
\hline Group & 2 & 1.522 & 0.236 & 0.101 \\
\hline Time $\times$ group & 2 & 3.171 & 0.05 & 0.19 \\
\hline
\end{tabular}

df, degrees of freedom; $\mathrm{p}^{2}$, partial eta-squared. *Pre, stm, post, post_24h; ${ }^{\dagger} \mathrm{Ml}$ or sham: ${ }^{*}$ resting MEP, active MEP or sham.

centered at $(-7,-38,75 \mathrm{~mm})$ in MNI coordinates-and the contralateral (contra) leg motor volume-sphere with a radius $1 \mathrm{~cm}$ centered at $(6,-38,75 \mathrm{~mm})$ in MNI coordinates - was extracted for analyzing the specificity and the average electric field unit vector.

The smaller active electrode showed a higher target area specificity (0.0311) than the bigger tDCS electrode $(0.0184)$.

\section{Discussion}

In the present study, the impact of tDCS on lower limb motor learning was studied in terms of accuracy and speed as performance components of a VMT. Our results show that anodal tDCS over the lower limb motor cortex representation has a significant long-term
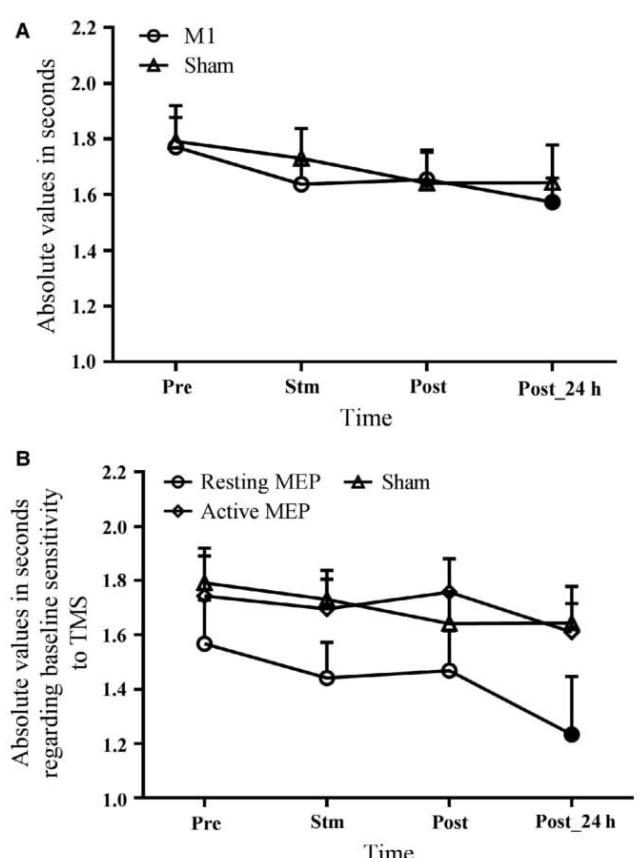

FIG. 2. Cursor time. Filled symbols indicate the difference from baseline values ( $P \leq 0.05$, two-tailed paired-samples $t$-test); (A) cursor time for the motor cortex stimulation group (M1) and for sham stimulation. (B) Cursor time results regarding baseline transcranial magnetic stimulation sensitivity. The error bars denote standard errors of the mean

effect on motor performance, that this effect critically depends on the sensitivity to TMS and might be affected by electrode size. Performance accuracy was improved during and immediately after tDCS in real and sham stimulation conditions; however, $24 \mathrm{~h}$ after stimulation the enhancement was significant only in the real stimulation condition. After $24 \mathrm{~h}$, we also observed reduced cursor time as compared to baseline values only in the real stimulation conditions. These results are indicative for offline consolidation effects of tDCS. 
8 A. Foerster et al.
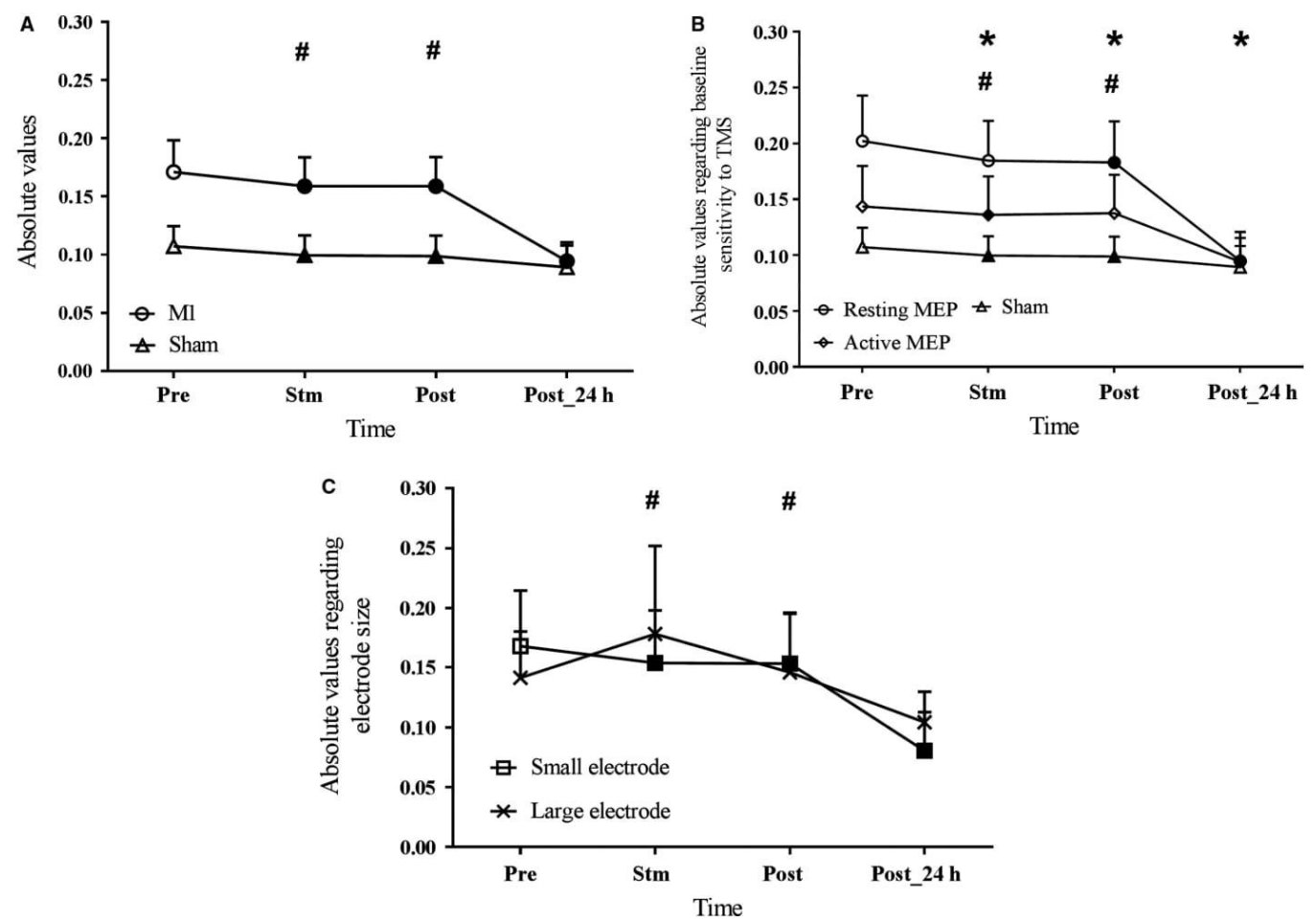

FIG. 3. Accuracy. Filled symbols indicate the difference from baseline values $(P \leq 0.05$, two-tailed paired-samples $t$-test); '\#' indicates significant differences of the 24-h post-intervention (post 24h) measures $(P \leq 0.05$, two-tailed paired-samples $t$-test) in resting motor evoked potential and small electrode groups. '** indicates significant differences from sham stimulation conditions $(P<0.05$, two-tailed independent-samples $t$-test). (A) Accuracy values for the motor cortex stimulation goup (M1) and for sham stimulation. (B) Accury results taking into account baseline transcranial magnetic stimulation sensitivity. (C) Accuracy results regarding electrode size. The error bars denote standard error of means.

TABLE 5. Results of the repeated-measures ANOVA performed for each outcome measure in the secondary experiment comparing different electrode sizes

\begin{tabular}{lllll}
\hline & df & $F$ & $P$ & $\mathrm{p \eta}^{2}$ \\
\hline $\begin{array}{l}\text { Performance speed } \\
\quad \text { Time* }\end{array}$ & 3 & 0.762 & 0.439 & 0.132 \\
$\quad$ Electrode size & & & & \\
$\quad$ Time $\times$ electrode size & 1 & 0.789 & 0.415 & 0.136 \\
$\quad$ Cursor time & 3 & 0.689 & 0.452 & 0.121 \\
$\quad$ Time & 3 & 0.56 & 0.65 & 0.101 \\
$\quad$ Electrode size & 1 & 1.623 & 0.259 & 0.245 \\
$\quad$ Time $\times$ electrode size & 3 & 0.787 & 0.52 & 0.136 \\
$\quad \begin{array}{l}\text { Accuracy } \\
\quad \text { Time }\end{array}$ & 3 & 8.456 & 0.023 & 0.628 \\
$\quad$ Electrode size & 1 & 0.01 & 0.924 & 0.002 \\
$\quad$ Time $\times$ electrode size & 3 & 1.491 & 0.257 & 0.23 \\
\hline
\end{tabular}

df, degrees of freedom; $\mathrm{p \eta}^{2}$, partial eta-squared. *Pre, stm, post, post_24h; small $\left(8 \mathrm{~cm}^{2}\right)$ or large $\left(35 \mathrm{~cm}^{2}\right)$ electrode.

Interestingly, the results show not only performance improvemen during learning, but also improved over night consolidation. Motor performance improvements can occur during training (online) but also after training ended (offline). Offline processes, including skill stabilization and improvement (Korman et al., 2003; Fischer et al., 2005), reflect motor memory consolidation (Muellbacher et al., 2002; Doyon \& Benali, 2005), an intermediate stage between fast learning and slow learning (Doyon et al., 2009). Online and offline improvements can be maintained over time, resulting in long-term retention (Romano et al., 2010).

The long-term retention effect on motor performance presented in our results is consistent with previous reports using similar paradigms for performance speed (Devanathan \& Madhavan, 2016) and accuracy (Shah et al., 2013; Sriraman et al., 2014). However, longterm effects on performance accuracy and speed were not monitored so far in the same study simultaneously. Previous studies have always investigated only one of these performance aspects.

In contrast to a previous study (Sriraman et al., 2014), online accuracy performance improvement was present in both experimental groups (anodal and sham tDCS), which suggests that the improvement was dominated by a task learning effect, which was not boosted online by the intervention. Experimental design differences might explain this discrepancy. In our study, we applied about $50 \%$ of current and total charge density applied in the other study, our stimulation was applied over the dominant motor cortex 
TABLE 6. Computational analysis based on the active tDCS electrode size (ML, medio-lateral; PA, posterior-anterior; IS, inferior-superior)

\begin{tabular}{|c|c|c|c|c|c|}
\hline \multirow[b]{2}{*}{$\begin{array}{l}\text { Active electrode } \\
\text { size/current intensity }\end{array}$} & \multicolumn{2}{|c|}{$\begin{array}{l}\text { Targeted leg motor cortex: sphere with a } \\
\text { radius of } 1 \mathrm{~cm} \text { centered at }(-7,-38, \\
75 \mathrm{~mm}) \text { in MNI coordinates }\end{array}$} & \multicolumn{2}{|c|}{$\begin{array}{l}\text { Contralateral leg motor cortex: sphere } \\
\text { with a radius } 1 \mathrm{~cm} \text { centered at }(6,-38, \\
75 \mathrm{~mm}) \text { in MNI coordinates }\end{array}$} & \multirow[b]{2}{*}{ Specificity } \\
\hline & $\begin{array}{l}\text { Electric } \\
\text { field }(\mathrm{V} / \mathrm{m})\end{array}$ & $\begin{array}{l}\text { Coefficient of } \\
\text { variation }\end{array}$ & $\begin{array}{l}\text { Electric } \\
\text { field }(\mathrm{V} / \mathrm{m})\end{array}$ & $\begin{array}{l}\text { Coefficient of } \\
\text { variation }\end{array}$ & \\
\hline \multicolumn{6}{|l|}{$8 \mathrm{~cm}^{2} / 0.5 \mathrm{~mA}$} \\
\hline ML & 0.3169 & 0.3195 & 0.4618 & 0.2221 & 0.0311 \\
\hline PA & -0.2609 & 0.2732 & -0.1566 & 0.1984 & \\
\hline IS & -0.9119 & 0.2428 & -0.8730 & 0.2412 & \\
\hline \multicolumn{6}{|l|}{$35 \mathrm{~cm}^{2} / 2 \mathrm{~mA}$} \\
\hline ML & 0.3378 & 0.3242 & 0.4194 & 0.1993 & 0.0184 \\
\hline PA & -0.1213 & 0.2426 & -0.0343 & 0.1868 & \\
\hline IS & -0.9334 & 0.2290 & -0.9072 & 0.2316 & \\
\hline
\end{tabular}

tDCS, transcranial direct current stimulation; MNI, Montreal Neurological Institute.

hemisphere, and the VMT was not identical between studies. It is known that for healthy volunteers, task difficulty and targeted hemisphere (dominant or non-dominant) affect motor performance results (Boggio et al., 2006). We stimulated the dominant hemisphere to achieve reliable hot spot determination for positioning of the smal tDCS electrode, considering that (i) TMS studies performed with the upper limb motor cortex showed that the dominant, compared to the non-dominant, motor cortex is characterized by having a larger motor evoked potential (De Gennaro et al., 2004), and (ii) the leg motor area is relatively inaccessible to TMS delivered over the scalp, as the lower limb motor cortex representation is deeply buried within the intercerebral fissure at $3-4 \mathrm{~cm}$ depth from the scalp surface (Terao et al., 2000).

Considering our results, the motor learning improvement observe in our study furthermore depends on the sensitivity to TMS. This is in accordance with previous studies, in which it was suggested that the neurophysiologic effect of anodal tDCS over the upper limb motor cortex is correlated with the sensitivity to TMS as well (Labruna et al., 2016; Jamil et al., 2017). The exact reasons for this fact await to be elucidated, but interindividual differences in the depth of respective cortical representations, which determine electrode/coil to target distance, and thus both, sensitivity to TMS and TDCS, as well as differences in neuronal orientation between individuals, might contribute. Other factors, which might impact on the efficacy of both, TMS and tDCS, such as neurotransmitter and modulator availability, might also contribute.

The results furthermore suggest an impact of electrode size on the behavioral effects of tDCS. One approach to increase the focality of tDCS is the use of electrodes with a smaller contact area $\left(1-8 \mathrm{~cm}^{2}\right)$ instead of the conventional relatively large patch electrodes $(20$ $35 \mathrm{~cm}^{2}$ ) (Guler et al., 2016). It has been shown by upper limb motor cortex studies that smaller stimulation electrodes and a functionally inert return electrode (in the sense of missing direct physiological effects under this electrode) enable a physiologically more selective stimulation (Miranda et al., 2006; Nitsche et al., 2007; Klooster et al., 2016). Our control experiment, in which we contrasted effects of small and large stimulation electrodes, suggests that electrode size is also relevant for behavioral tDCS effects. As revealed by the computational analysis, the small anode was more specific to the targeted leg motor volume when compared to the large anode. The modeling analysis showed that the overall direction of the electric field vector was comparable at the targeted and contralateral leg motor volume for both electrode designs; however, the specificity of the small anode was almost two times larger than that of the large anode (see Table 6). Thus, it might be speculated that by its impact also on the non-targeted contralateral motor cortex, the large electrode might have diminished the efficacy of tDCS to enhance excitability of the target area indirectly via inhibitory transcallosal connections.

Some limitations of the study should be taken into account. (i) The number of volunteers per condition $(n=9)$ who were assigned to the re-analysis of the data of the main experiment taking into account the TMS sensitivity as factor, and who participated in the control experiment $(n=6)$ with large vs. small electrodes was relatively low. (ii) No randomization was performed for the secondary experiment. (iii) The trendwise — although not significant—different accuracy results in the sham as compared to the real stimulation group at baseline could have resulted in a ceiling effect for the sham tDCS group. (iv) We did not explore physiological effects of stimulation in this study, which would have helped to clarify mechanistic aspects further. For future experiments in which the dependency of stimulation effects on baseline TMS sensitivity is a planned comparison, other measures, such as motor threshold, percentage of maximum stimulator output to receive a defined MEP amplitude or related parameters should be obtained to help to clarify mechanistic effects. (v) A crossover design of the main experiment would have reduced intersubject variability, strengthened the design and thus would be preferable in the future studies. (vi) TMS sensitivity data for the sham group are missing, the hot spot was not evaluated via TMS in this group, because they did not receive real tDCS, and thus exact location of the motor cortex electrode was not required. These missing data compromise between-group comparability; however, we think that the direct comparison between low and high TMS sensitivity subjects in the real stimulation group is of main importance, because it suggests different efficacy of tDCS based on TMS sensitivity.

Studies conducted with larger sample sizes, stronger tDCS current density and/or model-based different electrode positions in low-sensitivity TMS individuals, and including physiological measures are required to understand the mechanisms involved in tDCS effects, and interindividual differences, leading to optimize stimulation effects.

\section{Conclusion}

Our findings suggest that anodal tDCS over the lower limb motor cortex can improve visuo-motor task performance in healthy 
10 A. Foerster et al.

subjects and that these effects critically depend on individual characteristics, such as sensitivity to TMS, and stimulation protocol characteristics, such as electrode size. Considering the possibility of using tDCS as a rehabilitation tool for gait disorders, future studies are needed to improve our understanding of the physiological effects of tDCS over the lower limb motor cortex and to optimize stimulation protocols accordingly.

\section{Acknowledgements}

This work was supported by the German Academic Exchange Service (DAAD) and the Campus France (PROCOPE Mobility Grants).

\section{Conflict of interest}

The authors declare that they have no conflict of interest.

\section{Data accessibility}

Due to the demands of the local ethics committee, data are stored locally, but data are available upon request from the authors.

\section{Author contributions}

All authors have substantially contributed to the conception, design and interpretation of data for this work. $\mathrm{AF}$ carried out the experiments. $\mathrm{AF}, \mathrm{AD}$, MFK and MAN have substantially contributed to the analysis of this work $\mathrm{AF}, \mathrm{AD}$ and MAN have substantially contributed to the interpretation of data. All authors have also drafted the work and revised it critically with contribution related to author order. All authors have approved the final version prior to submission and are in agreement.

\section{Abbreviations}

ANOVA, analysis of variance; BET, brain extraction tool; CNS, central nervous system; COG, center of gravity; $\mathrm{CoV}$, coefficient of variation; DAQ, data acquisition; EEG, electroencephalography; $\overrightarrow{E F}$, electric field; FSL, functional magnetic resonance imaging of the brain software library; M1, primary moto cortex; MEP, motor evoked potential; MNI, Montreal Neurological Institute; MRI, magnetic resonance imaging: MVC, maximum voluntary contraction; NIC, Neuroelectrics Instrument Controller; NMDA, N-methyl-D-aspartate; RMSE, root mean square error; TA, tibialis anterior; tDCS, transcranial direc current stimulation; TMS, transcranial magnetic current stimulation; VMT, visuo-motor task.

\section{References}

Alkadhi, H., Crelier, G.R., Boendermaker, S.H., Golay, X., Hepp-Reymond, M.-C. \& Kollias, S.S. (2002) Reproducibility of primary motor corte somatotopy under controlled conditions. Am. J. Neuroradiol., 23, 1524 1532

van Asseldonk, E.H. \& Boonstra, T.A. (2016) Transcranial direct current stimulation of the leg motor cortex enhances coordinated motor outpu during walking with a large inter-individual variability. Brain Stimul., 9 $182-190$

Boggio, P.S., Castro, L.O., Savagim, E.A., Braite, R., Cruz, V.C., Rocha, R.R., Rigonatti, S.P., Silva, M.T. et al. (2006) Enhancement of non-dominant hand motor function by anodal transcranial direct current stimulation. Neurosci. Lett., 404, 232-236.

Bolognini, N., Pascual-Leone, A. \& Fregni, F. (2009) Using non-invasive brain stimulation to augment motor training-induced plasticity, $J$. Neuroeng. Rehabil., 6, 1

Brunoni, A.R., Amadera, J., Berbel, B., Volz, M.S., Rizzerio, B.G. \& Fregni, F. (2011) A systematic review on reporting and assessment of adverse effects associated with transcranial direct current stimulation. Int. J. Neuropsychop., 14, 1133-1145.

Cabral, M.E., Baltar, A., Borba, R., Galvão, S., Santos, L., Fregni, F. \& Monte-Silva, K. (2015) Transcranial direct current stimulation: before, durMonte-Silva, K. (2015) Transcranial direct current stimu.
Campanac, E. \& Debanne, D. (2007) Plasticity of neuronal excitability: Hebbian rules beyond the synapse. Arch. Ital. Biol., 145, 277-287.

De Gennaro, L., Cristiani, R., Bertini, M., Curcio, G., Ferrara, M., Fratello, F., Romei, V. \& Rossini, P.M. (2004) Handedness is mainly associated with an asymmetry of corticospinal excitability and not of transcallosa inhibition. Clin. Neurophysiol., 115, 1305-1312.

Devanathan, D. \& Madhavan, S. (2016) Effects of anodal tDCS of the lowe limb M1 on ankle reaction time in young adults. Exp. Brain Res., 234, 377-385

Doyon, J. \& Benali, H. (2005) Reorganization and plasticity in the adult brain during learning of motor skills. Curr. Opin. Neurobiol., 15, 161167.

Doyon, J., Korman, M., Morin, A., Dostie, V., Tahar, A.H., Benali, H. Karni, A., Ungerleider, L.G. et al. (2009) Contribution of night and day sleep vs. simple passage of time to the consolidation of moto sequence and visuomotor adaptation learning. Exp. Brain Res, 195 , $15-26$.

Dutta, A, Paulus, W \& Nitsche, M.A. (2014) Facilitating myoelectric-control with tansent healthy humans. J. Neuroeng. Rehabil., 11, 13. Fischer, S. Nitschke, M.F. Melchert, U.H., Erdmann, C. \& Born, J. (2005) Fischer, S., Nitschke, M.F., Melchert, U.H., Erdmann, C. \& Born, J. (2005)
Motor memory consolidation in sleep shapes more effective neuronal repMotor memory consolidation in sleep shapes

resentations. J. Neurosci., 25, 11248-11255.

Fleming, M.K., Pavlou, M., Newham, D.J., Sztriha, L. \& Teo, J.T. (2017) Fleming, M.K., Pavlou, M., Newham, D.J., Sztriha, L. \& Teo, J.T. (2017)
Non-invasive brain stimulation for the lower limb after stroke: what do we Non-invasive brain stimulation for the lower limb after stroke: what do wo
know so far and what should we be doing next? Disabil. Rehabil., 39, know so

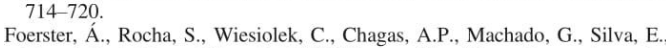
Fregni, F. \& Monte-Silva, K. (2013) Site-specific effects of mental practice combined with transcranial direct current stimulation on motor learning Eur. J. Neurosci., 37, 786-794

Guler, S., Dannhauer, M., Erem, B., Macleod, R., Tucker, D., Turovets, S. Luu, P., Erdogmus, D. et al. (2016) Optimization of focality and directio in dense electrode array transcranial direct current stimulation (tDCS). . Neural Eng., 13, 036020.

Hallett, M. (2003) Surround inhibition. Suppl. Clin. Neurophys., 56, 153159.

Hashemirad, F., Zoghi, M., Fitzgerald, P.B. \& Jaberzadeh, S. (2016) The effect of anodal transcranial direct current stimulation on motor sequence
learning in healthy individuals: a systematic review and meta-analysis. Brain Cogn., 102, 1-12.

Holmes, C.J., Hoge, R., Collins, L., Woods, R., Toga, A.W. \& Evans, A.C. (1998) Enhancement of MR images using registration for signal averaging. J. Comput. Assist. Tomogr., 22, 324-333.

J. Comput. Assiss. The (a) F.C. \& Cohen, L.G. (2006) Non-invasive brain stimulation: a new 708-712.

Jamil, A., Batsikadze, G., Kuo, H.I., Labruna, L., Hasan, A., Paulus, W. \& Nitsche, M.A. (2017) Systematic evaluation of the impact of stimulation intensity on neuroplastic after-effects induced by transcranial direct current intensity on neuroplastic after-effects incer
stimulation. J. Physiol. 595, 1273-1288.

Jayaram, G. \& Stinear, J.W. (2009) The effects of transcranial stimulation on paretic lower limb motor excitability during walking. J. Clin. Neurophysiol., 26, 272-279.

Jeffery, D.T., Norton, J.A., Roy, F.D. \& Gorassini, M.A. (2007) Effects of transcranial direct current stimulation on the excitability of the leg moto cortex. Exp. Brain Res., 182, 281-287.

Jenkinson, M., Beckmann, C.F., Behrens, T.E., Woolrich, M.W. \& Smith, S.M. (2012) Fsl. Neurolmage, 62, 782-790.

Jurcak, V., Tsuzuki, D. \& Dan, I. (2007) 10/20, 10/10, and 10/5 systems revisited: their validity as relative head-surface-based positioning systems. NeuroImage, 34, 1600-1611.

Kang, N., Summers, J.J. \& Cauraugh, J.H. (2016) Transcranial direct current stimulation facilitates motor learning post-stroke; a systematic review an meta-analysis. J. Neurol. Neurosurg. Psychiatry, 87, 345-355.

Klooster, D.C., de Louw, A.J., Aldenkamp, A., Besseling, R., Mestrom, R. Carrette, S., Zinger S., Bergmans, J.W et al. (2016) Technical aspects of neurostimulation focus on equipment, electric field modeling, and stimula tion protocols. Neurosci. Biobehav. Rev. 65, 113-141.

Korman, M. Raz N, Flash, T \& Karni, A, (2003) Multiple shifts in the Ko representation of a motor sequence during the acquisition of skilled perforKumru, H, Murillo, N, Benito-Penalva, J. Tormos, J.M \& Vidal, J. (2016)

Transcranial direct current stimulation is not effective in the motor strength 
and gait recovery following motor incomplete spinal cord injury during Lokomat $^{\oplus}$ gait training. Neurosci. Lett., 620, 143-147.

Labruna, L., Jamil, A., Fresnoza, S., Batsikadze, G., Kuo, M.-F., Vande schelden, B., Ivry, R.B. \& Nitsche, M.A. (2016) Efficacy of anodal transcranial direct current stimulation is related to sensitivity to transcranial magnetic stimulation. Brain Stimul., 9, 8-15.

Laczó, B., Antal, A., Rothkegel, H. \& Paulus, W. (2014) Increasing human leg motor cortex excitability by transcranial high frequency random noise stimulation. Restor. Neurol. Neuros., 32, 403-410.

Liebetanz, D., Nitsche, M.A., Tergau, F. \& Paulus, W. (2002) Pharmacological approach to the mechanisms of transcranial DC-stimulation-induce after-effects of human motor cortex excitability. Brain, 125, 2238-2247.

Madhavan, S. \& Shah, B. (2012) Enhancing motor skill learning with transcranial direct current stimulation - a concise review with applications to stroke. Front. Psychiatry, 3, 66

Madhavan, S. \& Stinear, JW. (2010) Focal and bidirectional modulation of lower limb motor cortex using anodal transcranial direct current stimulation. Brain Stimul., 3, 42-50.

Madhavan, S. Weber, K A \& Stinear, JW (2011) Non-invasive brain stimulation enhances fine motor control of the hemiparetic ankle: implications ulation enhances fine motor control of the hemi

Marquez, J., Conley, A., Karayanidis, F., Lagopoulos, J. \& Parsons, M. (2015)

Marquez, J., Conley, A., Karayanidis, F., Lagopoulos, J. \& Parsons, M. (2015)
Anodal direct current stimulation in the healthy aged: effects determined by Anodal direct current stimulation in the healthy aged: effects de
the hemisphere stimulated. Restor. Neurol. Neuros, 33, 509-519.

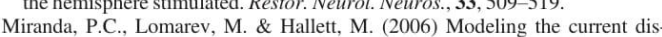
tribution during transcranial direct current stimulation. Clin. Neurophysiol., tribution during

Muellbacher, W., Ziemann, U., Wissel, J., Dang, N., Kofler, M., Facchini, S., Boroojerdi, B., Poewe, W. et al. (2002) Early consolidation in huma primary motor cotex $N, \mathbf{4 1 5}, 640-64$

Nair, D.G., Renga, V., Lindenberg, R., Zhu, L. \& Schlaug, G. (2011) Opt mizing recovery potential through simultaneous occupational therapy an non-invasive brain-stimulation using tDCS. Restor. Neurol. Neuros., 29, 411-420.

Nitsche, M.A., Doemkes, S., Karakose, T., Antal, A., Liebetanz, D., Lang. N., Tergau, F. \& Paulus, W. (2007) Shaping the effects of transcranial direct current stimulation of the human motor cortex. J. Neurophysiol., 97, 3109-3117.

Oostenveld, R. \& Praamstra, P. (2001) The five percent electrode system for high-resolution EEG and ERP measurements. Clin. Neurophysiol. 112. 713-719.

Otal, B., Dutta, A., Foerster, Á., Ripolles, O., Kuceyeski, A., Miranda, P.C. Edwards, D.J., Ilić, T.V. et al. (2016) Opportunities for guided multichannel non-invasive tic tion. Front. Neurol., 7, 21

Porter R \& Lemon, R (1993) Corticospinal Function and Voluntary MovePorter, R. \& Lemon, R. (1993). Corticospinal Fun
ment. Oxford University Press, New York, NY.
Rocha, S., Silva, E., Foerster, Á., Wiesiolek, C., Chagas, A.P., Machado, G., Baltar, A. \& Monte-Silva, K. (2016) The impact of transcrania direct current stimulation (tDCS) combined with modified constrain induced movement therapy (mCIMT) on upper limb function in chronic stroke: a double-blind randomized controlled trial. Disabil. Rehabil., 38 653-660.

Roche, N., Lackmy, A., Achache, V., Bussel, B. \& Katz, R. (2011) Effect of anodal transcranial direct current stimulation over the leg motor area o lumbar spinal network excitability in healthy subjects. J. Physiol., 589, 2813-2826.

Romano, J.C., Howard, J.H. Jr \& Howard, D.V. (2010) One-year retention of general and sequence-specific skills in a probabilistic, serial reaction time task. Memory, 18, 427-441.

Rossini, P.M., Barker, A., Berardelli, A., Caramia, M., Caruso, G., Cracco, R., Dimitrijević, M., Hallett, M. et al. (1994) Non-invasive electrical and magnetic stimulation of the brain, spinal cord and roots: basic principles and procedures for routine clinical application. Report of an IFCN committee. Electroencephalogr. Clin. Neurophysiol., 91, 79-92.

Shah, B. Nguyen, TT \& Madhavan, S. (2013) Polarity independent effects , B., Nguyen, T.T. \& Madhavan, S. (2013) Polarity independent efrects $066-968$.

Soekadar, S.R., Witkowski, M., Garcia Cossio, E., Birbaumer, N. \& Cohen, L. (2014) Learned EEG-based brain self-regulation of motor-related oscillations during application of transcranial electric brain stimulation: feasibility and limitations. Front. Behav. Neurosci., 8, 93.

Sriraman, A., Oishi, T. \& Madhavan, S. (2014) Timing-dependent priming effects of tDCS on ankle motor skill learning. Brain Res., 1581, 23-29. Stagg, C.J. \& Nitsche, M.A. (2011) Physiological basis of transcranial direct current stimulation. Neuroscientist, 17, 37-53.

Tanaka, S., Hanakawa, T., Honda, M. \& Watanabe, K. (2009) Enhancement of pinch force in the lower leg by anodal transcranial direct current stimulation. Exp. Brain Res., 196, 459-465.

Tanaka, S., Takeda, K., Otaka, Y., Kita, K., Osu, R., Honda, M., Sadato, N., Hanakawa, T. et al. (2011) Single session of transcranial direct current stimulation transiently increases knee extensor force in patients with hemiparetic stroke. Neurorehab. Neural Re., 25, 565-569.

Terao, Y., Ugawa, Y., Hanajima, R., Machii, K., Furubayashi, T., Mochizuki, H., Enomoto, H., Shiio, Y. et al. (2000) Predominant activation of I1-waves from the leg motor area by transcranial magnetic stimulation. Brain Res. 859, 137-146.

Windhoff, M., Opitz, A. \& Thielscher, A. (2013) Electric field calculations in brain stimulation based on finite elements: an optimized processing pipeline for the generation and usage of accurate individual head models. Hum. Brain Mapp., 34, 923-935.

Ziemann. U. Paulus, W., Nitsche, M.A. Pascual-Leone, A. Byblow, W.D.

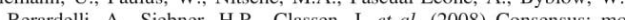
cortex plasticity protocols. Brain Stimul., 1, 164-182. 


\subsection{Cerebellar Transcranial Direct Current Stimulation (ctDCS) Impairs Balance Control in Healthy Individuals}

The cerebellum is well known to play an important role in movement execution and motor control by modulation of the primary motor cortex (M1) through cerebellothalamocortical connections (160). There is a concensus that tDCS can effectively influence cerebellar functions in the motor domain, with effects on visually guided tracking tasks, motor surround inhibition, motor adaptation and learning (161). In this study, we aimed to investigate the effects of cerebellar tDCS (ctDCS) on postural balance in healthy individuals. Fifteen healthy and right-handed subjects were submitted to three sessions of ctDCS (anodal, cathodal and sham), separated by at least $48 \mathrm{~h}$. In each session, tests of static (right and left Athlete Single Leg tests) and dynamic balance (Limits of Stability test) were performed using the Biodex Balance System before and immediately after ctDCS. The results revealed that cathodal ctDCS impaired static balance of healthy individuals, reflected in higher scores on the overall stability index when compared to baseline for right $(p=0.034)$ and left $(p=$ 0.01) Athlete Single Leg test. In addition, we found a significant impairment for the left Athlete Single Leg test in comparison to sham stimulation $(p=0.04)$. As far as we know, this is the first study that shows changes of balance control after ctDCS in healthy individuals. This finding raises insights useful for further investigations of cerebellar modulation in neurological patients. 


\title{
Cerebellar Transcranial Direct Current Stimulation (ctDCS) Impairs Balance Control in Healthy Individuals
}

\author{
Águida Foerster ${ }^{1,2} \cdot$ Lorena Melo $^{1} \cdot$ Marina Mello $^{1} \cdot$ Rebeca Castro $^{1} \cdot$ Lívia Shirahige $^{1} \cdot$ \\ Sérgio Rocha ${ }^{1} \cdot$ Kátia Monte-Silva ${ }^{1}$
}

Abstract The cerebellum plays an important role in the planning, initiation and stability of movements, as well as in postural control and balance. Modulation of neural regions underlying balance control may be a potential alternative to treat balance impairments in cerebellar patients. Transcranial direct current stimulation (tDCS) is a noninvasive and safe tool capable to modulate cerebellar activity. We aim to investigate the effects of cerebellar tDCS (ctDCS) on postural balance in

\begin{tabular}{|c|c|}
\hline & uida Foerster and Lorena Melo contributed equally to this work. \\
\hline & $\begin{array}{l}\text { ctronic supplementary material The online version of this article } \\
\text { i: } 10.1007 / \mathrm{s} 12311-017-0863-8 \text { ) contains supplementary material, } \\
\text { ich is available to authorized users. }\end{array}$ \\
\hline 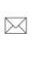 & $\begin{array}{l}\text { Kátia Monte-Silva } \\
\text { monte.silvakk@gmail.com }\end{array}$ \\
\hline & $\begin{array}{l}\text { Águida Foerster } \\
\text { foerster.as@gmail.com }\end{array}$ \\
\hline & $\begin{array}{l}\text { Lorena Melo } \\
\text { lorenafdemelo@gmail.com }\end{array}$ \\
\hline & $\begin{array}{l}\text { Marina Mello } \\
\text { marina_mello@hotmail.com }\end{array}$ \\
\hline & $\begin{array}{l}\text { Rebeca Castro } \\
\text { beck.castro@gmail.com }\end{array}$ \\
\hline & $\begin{array}{l}\text { Lívia Shirahige } \\
\text { livia.sgn@gmail.com }\end{array}$ \\
\hline & $\begin{array}{l}\text { Sérgio Rocha } \\
\text { srocha3105@gmail.com }\end{array}$ \\
\hline 1 & $\begin{array}{l}\text { Physical Therapy Department, Applied Neuroscience Laboratory, } \\
\text { Universidade Federal de Pernambuco, Avenida Jornalista Aníbal } \\
\text { Fernandes, Recife 50670-900, Brazil }\end{array}$ \\
\hline & $\begin{array}{l}\text { Leibniz Research Centre for Working Environment and Human } \\
\text { Factors, Dortmund, Germany }\end{array}$ \\
\hline
\end{tabular}

Published online: 29 April 2017 healthy individuals. Fifteen healthy and right-handed subjects were submitted to three sessions of ctDCS (anodal, cathodal and sham), separated by at least $48 \mathrm{~h}$. In each session, tests of static (right and left Athlete Single Leg tests) and dynamic balance (Limits of Stability test) were performed using the Biodex Balance System before and immediately after the ctDCS. The results revealed that cathodal ctDCS impaired static balance of healthy individuals, reflected in higher scores on overall stability index when compared to baseline for right $(p=0.034)$ and left $(p=0.01)$ Athlete Single Leg test. In addition, we found significant impairment for left Athlete Single Leg test in comparison to sham stimulation $(p=0.04)$. As far as we know, this is the first study that points changes on balance control after ctDCS in healthy individuals. This finding raises insights to further investigation about cerebellar modulation for neurological patients.

Keywords Transcranial direct current stimulation (tDCS) . Cerebellum $\cdot$ Postural balance

\section{Introduction}

The cerebellum is a complex structure that has extensive connections to many areas of the brainstem, midbrain, and cerebral cortex. The connections with the vestibular nuclei and the vestibular apparatus give to the cerebellum a crucial role in the maintenance of equilibrium and the coordination of head and eye movements [1].

Recently, cerebellar activity has been modulated through noninvasive cerebellar stimulation techniques and provided novel information about its functions [2]. Indeed, several studies using cerebellar transcranial direct current stimulation (ctDCS) have shed light on the influence of the cerebellum on motor learning $[3,4]$. For example, Jayaram and colleagues

Springer SRC 
[5] have found that anodal cerebellar tDCS applied during walking improved locomotor learning in healthy subjects, while cathodal stimulation disrupted it. This finding suggests that cerebellar stimulation techniques could be used as a neurorehabilitation tool within the context of locomotor training for patients with gait impairments.

However, it remains unknown whether ctDCS can also effectively influence balance control and a better understanding of how ctDCS affects balance may help in its application to clinical practice in the future. Thus, the main goal of this study was to investigate the effects of ctDCS on static and dynamic balance in healthy individuals.

\section{Material and Methods}

Fifteen healthy right-handed (assessed by Edinburgh Handedness Inventory) [6] and right-footed (self-report) females (aged 21-24 years) were enrolled to the study. None of the participants had balance impairments (Berg Balance Scale score $=56$ ) and were taking neuroactive substances or medication during the experiment or presented any exclusion criteria for tDCS [7]. All volunteers gave their consent prior to the experiment and procedures were approved by the University Research Ethics Committee

A randomized, controlled, double-blinded crossover design (counterbalanced order) was conducted in three experimental sessions. A noninvolved researcher used a specific procedure (www.randomization.com) to perform randomization. After, allocation assignments were kept in opaque-sealed envelopes and handled only by the investigator responsible to apply cerebellar stimulations. Volunteers and evaluators were blinded to ctDCS modality.

For balance assessment, the Biodex Balance System (BBS) platform (Biodex Medical Systems, EUA) was used, based on previous studies $[8,9]$. The platform has various levels of stability (range from 1 -lowest stability to 12 - greatest stability). Static balance was analyzed by Athlete Single Leg tests (ASL) at the level 6 of stability. For this test, the participants were instructed to maintain the center of mass (CoM) as static as possible for $20 \mathrm{~s}$, receiving feedback provided by the platform screen. In addition, the volunteers were required to stand on their dominant (RightASL) and then, on their nondominant leg (LeftASL). For each test, an overall stability index (OSI) was generated by BBS. The OSI represents the angular excursion of the volunteer's center of gravity during the test. A lower OSI is indicative of few movements and greater ability in balance maintenance.

In order to evaluate dynamic balance, the Limits of Stability test (LS) was performed with the platform at level 12 of stability. For this test, the participants were instructed to stand on the platform and lean in eight directions (forward, backward, right, left, forward/right, forward/left, backward/ right and backward/left) to make a cursor displayed on the platform screen hit a target for $20 \mathrm{~s}$. At the end of testing, BBS provides also a score (OSI) that represents subjects' ability to accurately move the cursor to the target in all eight directions. For this test, higher OSI indicates better balance control. Subjects' feet were positioned to maintain CoM over the center of the platform, and the coordinates were recorded to maintain the same feet position throughout the sessions. Participants were not allowed to grasp the handles of the platform. All tests were performed before and immediately after ctDCS.

To apply ctDCS, direct current ( $1 \mathrm{~mA}$ ) was delivered by an electrical stimulator (Soterix, USA) through a pair of salinesoaked sponge electrodes $\left(25 \mathrm{~cm}^{2}\right)$. Anodal, cathodal and sham ctDCS were applied in different sessions, separated by at least $48 \mathrm{~h}$. The electrodes were placed on the right cerebellar hemisphere ( $3 \mathrm{~cm}$ lateral to the inion) and over the deltoid muscle in the right arm. Anodal ctDCS was performed for $13 \mathrm{~min}$ while cathodal stimulation, for $9 \mathrm{~min}$. These protocols have been widely used [10]. Sham ctDCS was applied only for $30 \mathrm{~s}$ [7], but the volunteers remained with electrodes montage for $13 \mathrm{~min}$. After ctDCS, presence of adverse effects was analyzed.

\section{Data Analysis}

A Shapiro-Wilk test was performed to analyze data distribution. Repeated-measures ANOVAs $(3 \times 2)$ were performed for each measure (RightALS, LeftALS and LS) considering within-subjects factors "ctDCS" (cathodal, anodal and sham ctDCS) and "time" (before and after ctDCS). Post hoc twotailed paired samples $t$ tests were used when necessary (not adjusted for multiple comparisons). Significance level was set to $\alpha<5 \%$. Data was analyzed using SPSS version 20.0 for Windows.

\section{Results}

No difference was found among sessions for any baseline balance measure. Analyzing OSI scores, the ANOVA revealed significant effects for RightASL (time: F:10.174, $p=0.008$ ) and LeftASL (time*ctDCS: $F=4.678$, $p=0.035$ ). For RightASL, post hoc $t$ test demonstrated a significant increase of OSI after cathodal ctDCS when compared to baseline $(t=-2.353, p=0.034)$. Likewise, post hoc test showed an increase of OSI after cathodal ctDCS when compared to baseline $(t=-2.978, p=0.01)$ and to sham stimulation $(t=2.177, p=0.04)$, reflecting an impairment of static balance in both tests (Fig. 1 and Table S1). We did not find any differences on adverse effects between groups (Table 1). 
Fig. 1 Illustration of subject position (left panel) on the platform of Biodex Balance System during the performance of right (a) and left (b) athlete single tests and limits of stability test (c). Trajectory of center of mass from Trajectory of center of mass from
one participant during the tests (middle panel). Mean and standard deviation of stability scores (right panel) before (white bars) and immediately after (black bars) sham, cathodal and anodal cerebellar transcranial direct stimulation (ctDCS). *Significant difference from baseline and ${ }^{\#}$ from sham stimulation $(p<0.05$, two-tailed paired $t$ test)
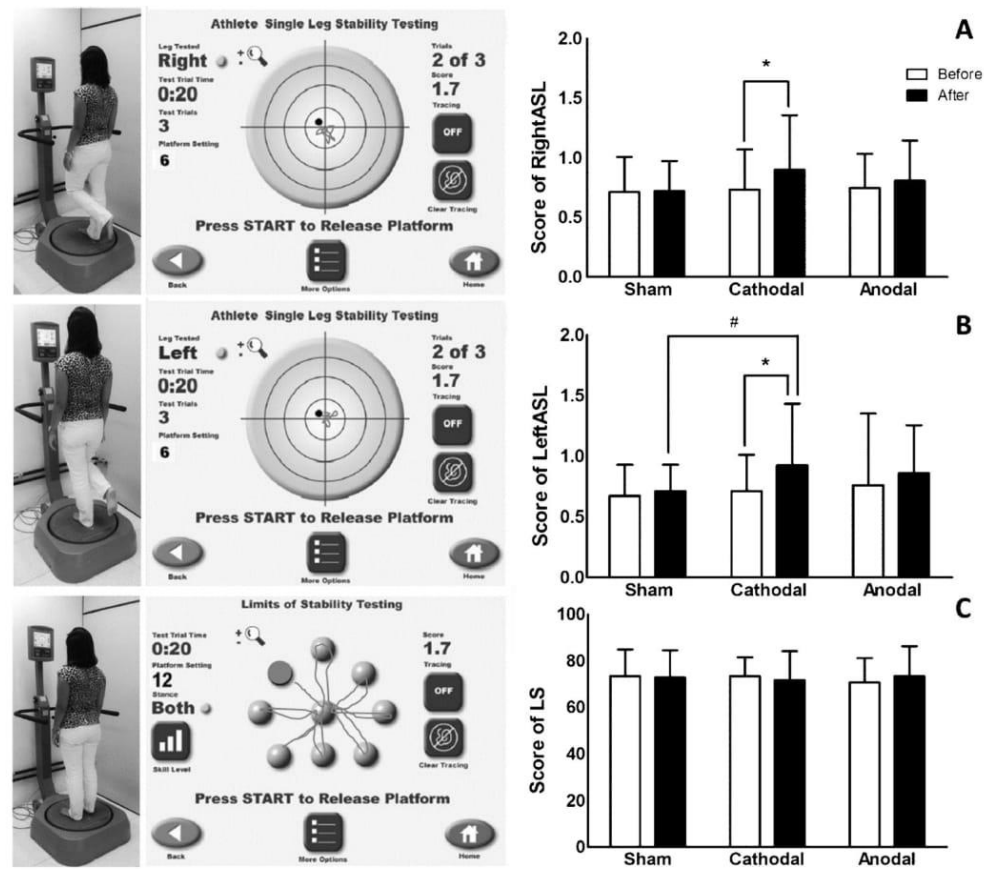

\section{Discussion}

The main finding of the present study is that ctDCS was able to interfere with static balance in healthy individuals and seems to be a safe tool to modulate the cerebellum's activity. Cathodal tDCS over the right cerebellar hemisphere impaired static balance control in the right and left single-limb stances,

reflected by higher scores at RightASL and LeftASL. Similarly to our results, some studies have also found significant impairment of the cerebellar functions, such as motor learning [11, 12] after cathodal ctDCS.

The mechanisms underlying the negative effects of cathodal ctDCS on static balance are not clear. It is possible that cathodal ctDCS decreases the responsiveness of the cerebellar

Table 1 Percentage of volunteers who reported adverse effects during or after ctDCS

\begin{tabular}{lcccc}
\hline Adverse effect & Sham ctDCS & Anodal ctDCS & Cathodal ctDCS & $p$ \\
\hline Headache (\%) & 13.3 & 6.7 & 0 & 0.34 \\
Neck pain (\%) & 0 & 0 & 6.7 & 0.36 \\
Scalp pain (\%) & 0 & 0 & 0 & $\#$ \\
Tingling (\%) & 13.3 & 20 & 0 & 0.20 \\
Itching (\%) & 20 & 13.3 & 13.3 & 0.84 \\
Burning (\%) & 0 & 0 & 0 & $\#$ \\
Skin redness (\%) & 6.7 & 13.3 & 0 & 0.34 \\
Sleepiness (\%) & 6.7 & 20 & 26.7 & 0.34 \\
Trouble concentrating (\%) & 0 & 0 & 6.7 & 0.36 \\
Acute mood change (\%) & 0 & 0 & 0 & $\#$ \\
Others (\%) & 0 & 0 & 0 & $\#$ \\
\hline
\end{tabular}

Chi-square test; \# statistic was not computed due to the concentration of data being a constant 
neurons [13], apparently inducing a "virtual lesion." Given the evidence regarding the role of the cerebellum in balance control, mainly in one-foot stance [14], it seems likely that the decrease of cerebellar activity by cathodal ctDCS would affect the ability of the cerebellum to respond to postural adjustment when standing on one-foot, and it may underlie the impairment in balance control. However, we did not find an effect of ctDCS on dynamic balance in a bipodal support. This might indicate that this posture could require less cerebellar activation to balance adjustment than one-foot stance in healthy subjects. The cerebellum is widely known to participate on motor control receiving and sending information by afferent and efferent connections with contralateral motor cortex [1] Thus, the decrease in cerebellar activity after cathodal ctDCS may have induced an impairment of ipsilateral limb performance.

In addition, our findings on the left cerebellar hemisphere could be related to the connections between "motor" cerebellar lobules (HV, HVI, HVIIb, HVIII) and contralateral moto areas, such as greater coactivation of lobules HVIIb and HVIII with the left thalamus (prefrontal region and parietal projections) [15]. Moreover, we must consider that neuronal activity in supratentorial regions could be indirectly modulated by ctDCS.

One methodological limitation of our study was the application of ctDCS on one of the cerebellar hemispheres (right), instead of the cerebellar vermis, which is thought to be responsible for the control of upright posture during standing [1] Further studies should evaluate site-dependent and longlasting effects of tDCS over the cerebellum on equilibrium control.

To the best of our knowledge, this is the first study that demonstrated changes on postural balance after ctDCS. In conclusion, our findings suggest that cathodal ctDCS impairs static balance maintenance in healthy subjects. However, it is reasonable to assume that ctDCS was able to modulate a cerebellar function and those findings raise insights to further investigation about how cerebellar modulation could interfere with cerebellar motor functions.

Compliance with Ethical Standards

Conflict of Interest The authors declare that they have no conflict of interest.

Funding No funding was received for this study. Kátia Monte-Silva is supported by CNPq.

\section{References}

1. Barlow JS. The cerebellum and adaptive control. Cambridge: Cambridge University Press; 2002.

2. Fernandez L, Albein-Urios N, Kirkovski M, McGinley JL, Murphy AT, Hyde C, Stokes MA, Rinehart NJ, Enticott PG. Cathodal transcranial direct current stimulation (tDCS) to the right cerebellar hemisphere affects motor adaptation during gait. Cerebellum. 2016; doi: 10.1007/s12311-016-0788-7.

3. Ferrucci R, Brunoni AR, Parazzini M, Vergari M, Rossi E, Fumagalli M, Mameli F, Rosa M, Giannicola G, Zago S, Priori A. Modulating human procedural learning by cerebellar transcranial direct current stimulation. Cerebellum. 2013;12:485-92. doi:10. 1007/s12311-012-0436-9.

4. Steiner KM, Enders A, Thier W, Batsikadze G, Ludolph N, Ilg W, Timmann D. Cerebellar tDCS does not improve learning in a complex whole body dynamic balance task in young healthy subjects. PLoS One. 2016;11:e0163598. doi:10.1371/journal.pone.0163598.

5. Jayaram G, Tang B, Pallegadda R, Vasudevan EV, Celnik P, Bastian A. Modulating locomotor adaptation with cerebellar stimulation. J Neurophysiol. 2012;107:2950-7. doi:10.1152/jn.00645.2011.

6. Oldfield RC. The assessment and analysis of handedness: the Edinburgh inventory. Neuropsychologia. 1971;9:97-113.

7. Nitsche MA, Cohen LG, Wassermann EM, Priori A, Lang N, Antal A, Paulus W, Hummel F, Boggio PS, Fregni F. Transcranial direct current stimulation: state of the art2008. Brain stimulation. 2008;1: 206-23.

8. Paterno MV, Myer GD, Ford KR, Hewett TE. Neuromuscular training improves single-limb stability in young female athletes. The Journal of orthopaedic and sports physical therapy. 2004;34:30516. doi:10.2519/jospt.2004.34.6.305

9. Soysal Tomruk M, Uz MZ, Kara B, Idiman E. Effects of Pilates exercises on sensory interaction, postural control and fatigue in patients with multiple sclerosis. Multiple sclerosis and related disorders. 2016;7:70-3. doi:10.1016/j.msard.2016.03.008.

10. Nitsche MA, Paulus W. Sustained excitability elevations induced by transcranial DC motor cortex stimulation in humans. Neurology. 2001;57:1899-901.

11. Zuchowski ML, Timmann D, Gerwig M. Acquisition of conditioned eyeblink responses is modulated by cerebellar tDCS. Brain stimulation. 2014;7:525-31. doi:10.1016/j.brs.2014.03.010.

12. Herzfeld DJ, Pastor D, Haith AM, Rossetti Y, Shadmehr R, O'Shea J. Contributions of the cerebellum and the motor cortex to acquisition and retention of motor memories. Neurolmage. 2014;98:14758. doi:10.1016/j.neuroimage.2014.04.076.

13. Galea JM, Jayaram G, Ajagbe L, Celnik P. Modulation of cerebella excitability by polarity-specific noninvasive direct current stimulation. The Journal of neuroscience : the official journal of the Society for Neuroscience. 2009;29:9115-22. doi:10.1523/JNEUROSCI. 2184-09.2009.

14. Ouchi Y, Okada H, Yoshikawa E, Nobezawa S, Futatsubashi M. Brain activation during maintenance of standing postures in humans. Brain : a journal of neurology. 1999;122(Pt 2):329-38.

15. Balsters JH, Laird AR, Fox PT, Eickhoff SB. Bridging the gap between functional and anatomical features of cortico-cerebellar circuits using meta-analytic connectivity modeling. Hum Brain Mapp. 2014;35:3152-69. doi:10.1002/hbm.22392. 


\section{Chapter 3- Summary}

\subsection{General remarks}

The studies included in this thesis explored the impact of tDCS applied over the lower limb motor cortex and cerebellum on motor learning and cortical excitability in humans. In the first study, our results showed that a small-anode $(3.5 \mathrm{~cm} \times 1 \mathrm{~cm})$ with the same current density at the electrode surface as a large-anode $(5 \mathrm{~cm} \times 7 \mathrm{~cm})$ resulted in similar cortical excitability alterations of the targeted leg motor cortex respesentation, and that the small anode condition with the cathode placed over T7 resulted in the best stimulation specificity. In the second study, our results showed that anodal tDCS applied over lower limb M1 modulates VMT performance in healthy subjects, and the stimulation effects critically depend on sensitivity to TMS and electrode size. In the third study, static balance was impaired by cathodal cerebellar tDCS. These findings add important information to our understanding of the mechanisms of tDCS on lower limb motor functions, including neuroplasticity, motor learning, and the impact of the cerebellum on balance.

\subsection{Functional implications}

Our findings confirm that, in healthy humans, tDCS impacts lower limb motor cortex and cerebellar excitability, and motor performance. For the field of clinical application, the results suggest that tDCS might have therapeutic effects on lower limb functions via enhancing motor performance by plasticity induction, and that cerebellar stimulation might be suited to alter balance control.

The general interest to understand the mechanisms, and effects of tDCS applied over the lower limb M1 is growing. Studies in healthy humans $(15,16,162-$ 166) and in stroke patients $(16,45,48,167)$ showed evidence for excitability- 
enhancing and performance-improving effects of anodal tDCS over the lower limb motor cortex of humans.

However, missing knowledge about protocols inducing optimal tDCS effects hinders the use of tDCS as an adjuvant therapy aimed to improve lower limb motor functions. Regarding optimization of tDCS effects, it was recently shown that timing of stimulation relative to task performance is relevant, with better results when stimulation is applied during task performance (166). Our results add the information that the size of the target electrode, placement of the return electrode, and cortical baseline excitability are factors that should be taken into account for optimization of protocols when tDCS is applied over the lower limb motor cortex. Considering the possibility of using tDCS as a rehabilitation tool for gait disorders, future studies are needed to improve our understanding of the physiological effects of tDCS over the lower limb motor cortex, and to optimize stimulation protocols accordingly.

\subsection{Limitations}

Some potential limitations of the present work should be taken into account. First, we did not investigate direct neurophysiological effects of tDCS in our second and third studies, which would have enabled us to make a direct correlation between neuroplasticity and motor performance or balance control improvement observed in our results. Moreover, all studies in the thesis were conducted in healthy subjects. In neurological patients, brain function and reaction to stimulation might be different. However, due to the limited time frame, we did not have the chance to explore our results in neurological patients with lower limb motor impairment, thus presumed functional implications are speculative at present. 


\subsection{Future perspectives}

Our studies explored the impact of tDCS applied over the lower limb M1 and cerebellum on motor learning, cortical excitability, and corporal balance control in healthy humans. The results supply clear evidence for the relevance of tDCS to promote alterations of excitability of cortical representations of the lower limb and motor functions. Future studies should explore the mechanisms of action of tDCS applied over the lower limb M1 and cerebellum in larger detail, regarding stimulation parameters, electrode configuration, and neurophysiological outcomes in healty humans and in neurological patients.

The ability to walk is one of the most important motor functions performed by the lower limbs, and this motor activity plays a big role for performance of activities of daily living and therefore determines quality of life. At present, a couple of studies are available, which showed that tDCS has an impact on the excitability of cortical representations of the lower limbs, and lower limb motor function in chronic stroke patients. So far it was shown that (i) anodal stimulation over the ipsilesional motor cortex increased paretic limb and decreased nonparetic limb motor excitability (45); (ii) a single session of anodal tDCS over the paretic lower limb motor cortex representation increased knee extensor force in patients with hemiparetic stroke for up to 30 minutes following intervention (167); and (iii) anodal tDCS over the lesioned hemisphere showed beneficial effects on coordinated motor output during walking with however large inter-individual variability (48). Considering the possibility of using tDCS as a rehabilitation tool for gait disorders, future studies exploring the association between neuroplasticity, cortical excitability, motor performance, and functional outcome are needed to improve our understanding of the physiological 
effects of tDCS over the lower limb motor cortex, and to optimize stimulation protocols accordingly.

Overall, improved knowledge about the mechanisms of neuroplasticity and excitability of the human brain will strengthen the possibility to shape the plastic potential of the brain, and might open a broader field of new therapeutic and research perspectives. However, we are still at the beginning of our understanding of the neurophysiological and functional effects of neuromodulatory brain stimulation techniques (e.g. tDCS) on the human central motor nervous system. The central command of motor performance involves a complex brain network, and knowledge about how to strenghten this network in health and disease makes related studies important for improving our understanding of brain functions, but also for development of new therapeutic strategies to treat people suffering from diseases involving pathological alterations of motor functions. 


\section{References}

1. Drew T, Prentice S, Schepens B. Cortical and brainstem control of locomotion. Progress in brain research. 143: Elsevier; 2004. p. 251-61.

2. Hallett M. Plasticity of the human motor cortex and recovery from stroke. Brain research reviews. 2001;36(2-3):169-74.

3. Cramer SC, Sur M, Dobkin BH, O'brien C, Sanger TD, Trojanowski JQ, et al. Harnessing neuroplasticity for clinical applications. Brain. 2011;134(6):1591-609.

4. Ziemann U, Siebner HR. Modifying motor learning through gating and homeostatic metaplasticity. Brain stimulation. 2008;1(1):60-6.

5. Nitsche MA, Cohen LG, Wassermann EM, Priori A, Lang N, Antal A, et al. Transcranial direct current stimulation: state of the art 2008. Brain Stimulation. 2008;1(3):206-23.

6. Kumru H, Murillo N, Benito-Penalva J, Tormos JM, Vidal J. Transcranial direct current stimulation is not effective in the motor strength and gait recovery following motor incomplete spinal cord injury during Lokomat ${ }^{\circledR}$ gait training. Neuroscience letters. 2016;620:143-7.

7. Campanac E, Debanne D. Plasticity of neuronal excitability: Hebbian rules beyond the synapse. Archives italiennes de biologie. 2007;145(3):277-87.

8. Bolognini N, Pascual-Leone A, Fregni F. Using non-invasive brain stimulation to augment motor training-induced plasticity. Journal of neuroengineering and rehabilitation. 2009;6(1):8.

9. Foerster Á, Rocha S, Wiesiolek C, Chagas AP, Machado G, Silva E, et al. Site-specific effects of mental practice combined with transcranial direct current stimulation on motor learning. European journal of neuroscience. 2013;37(5):786-94.

10. Cabral ME, Baltar A, Borba R, Galvão S, Santos L, Fregni F, et al. Transcranial direct current stimulation: before, during, or after motor training? Neuroreport. 2015;26(11):618-22.

11. Hashemirad F, Zoghi M, Fitzgerald PB, Jaberzadeh S. The effect of anodal transcranial direct current stimulation on motor sequence learning in healthy individuals: a systematic review and metaanalysis. Brain and cognition. 2016;102:1-12.

12. Nair DG, Renga V, Lindenberg R, Zhu L, Schlaug G. Optimizing recovery potential through simultaneous occupational therapy and non-invasive brain-stimulation using tDCS. Restorative neurology and neuroscience. 2011;29(6):411-20.

13. Kang EK, Paik N-J. Effect of a tDCS electrode montage on implicit motor sequence learning in healthy subjects. Exp Transl Stroke Med. 2011;3(4).

14. Rocha S, Silva E, Foerster Á, Wiesiolek C, Chagas AP, Machado G, et al. The impact of transcranial direct current stimulation (tDCS) combined with modified constraint-induced movement therapy (mCIMT) on upper limb function in chronic stroke: a double-blind randomized controlled trial. Disability and rehabilitation. 2016;38(7):653-60.

15. Jeffery DT, Norton JA, Roy FD, Gorassini MA. Effects of transcranial direct current stimulation on the excitability of the leg motor cortex. Experimental brain research. 2007;182(2):281-7.

16. Tanaka S, Hanakawa T, Honda M, Watanabe K. Enhancement of pinch force in the lower leg by anodal transcranial direct current stimulation. Experimental Brain Research. 2009;196(3):459-65.

17. Madhavan S, Weber KA, Stinear JW. Non-invasive brain stimulation enhances fine motor control of the hemiparetic ankle: implications for rehabilitation. Experimental brain research. 2011;209(1):9-17.

18. Fleming MK, Pavlou M, Newham DJ, Sztriha L, Teo JT. Non-invasive brain stimulation for the lower limb after stroke: what do we know so far and what should we be doing next? Disability and rehabilitation. 2017;39(7):714-20.

19. Tucker MR, Olivier J, Pagel A, Bleuler H, Bouri M, Lambercy O, et al. Control strategies for active lower extremity prosthetics and orthotics: a review. Journal of neuroengineering and rehabilitation. 2015;12(1):1.

20. Capaday $C$. The special nature of human walking and its neural control. Trends in neurosciences. 2002;25(7):370-6. 
21. Nielsen JB, Sinkjær T. Afferent feedback in the control of human gait. Journal of electromyography and kinesiology. 2002;12(3):213-7.

22. Pons J, Moreno J, Torricelli D, Taylor J, editors. Principles of human locomotion: a review. Engineering in medicine and biology society (EMBC), 2013 35th annual international conference of the IEEE; 2013: IEEE.

23. Squire L, Berg D, Bloom FE, Du Lac S, Ghosh A, Spitzer NC. Fundamental neuroscience: Academic Press; 2012.

24. Sherrington CS. Remarks on some aspects of reflex inhibition. Proc R Soc Lond B. 1925;97(686):519-45.

25. Takahara D, Inoue Ki, Hirata Y, Miyachi S, Nambu A, Takada M, et al. Multisynaptic projections from the ventrolateral prefrontal cortex to the dorsal premotor cortex in macaquesanatomical substrate for conditional visuomotor behavior. European Journal of Neuroscience. 2012;36(10):3365-75.

26. Banich MT, Mackiewicz KL, Depue BE, Whitmer AJ, Miller GA, Heller W. Cognitive control mechanisms, emotion and memory: a neural perspective with implications for psychopathology. Neuroscience \& Biobehavioral Reviews. 2009;33(5):613-30.

27. Miller EK. The prefontral cortex and cognitive control. Nature reviews neuroscience. 2000;1(1):59.

28. Owen AM, McMillan KM, Laird AR, Bullmore E. N-back working memory paradigm: A meta-analysis of normative functional neuroimaging studies. Human brain mapping. 2005;25(1):4659.

29. Schweizer S, Grahn J, Hampshire A, Mobbs D, Dalgleish T. Training the emotional brain: improving affective control through emotional working memory training. Journal of Neuroscience. 2013;33(12):5301-11.

30. Staab JP, Balaban CD, Furman JM, editors. Threat assessment and locomotion: clinical applications of an integrated model of anxiety and postural control. Seminars in neurology; 2013: Thieme Medical Publishers.

31. Moon Y, Sung J, An R, Hernandez ME, Sosnoff JJ. Gait variability in people with neurological disorders: a systematic review and meta-analysis. Human movement science. 2016;47:197-208.

32. Amos A, Armstrong D, Marple-Horvat D. Changes in the discharge patterns of motor cortical neurones associated with volitional changes in stepping in the cat. Neuroscience letters. 1990;109(12):107-12.

33. Armstrong DM. The supraspinal control of mammalian locomotion. The Journal of physiology. 1988;405(1):1-37.

34. Beloozerova I, Sirota M. The role of the motor cortex in the control of accuracy of locomotor movements in the cat. The Journal of Physiology. 1993;461(1):1-25.

35. Drew $T$, Jiang $W$, Kably $B$, Lavoie $S$. Role of the motor cortex in the control of visually triggered gait modifications. Canadian journal of physiology and pharmacology. 1996;74(4):426-42. 36. Huda S, Rodriguez R, Lastra L, Warren M, Lacourse MG, Cohen MJ, et al. Cortical activation during foot movements: Il effect of movement rate and side. Neuroreport. 2008;19(16):1573-7.

37. Orr EL, Lacourse MG, Cohen MJ, Cramer SC. Cortical activation during executed, imagined, and observed foot movements. Neuroreport. 2008;19(6):625-30.

38. Ciccarelli O, Toosy A, Marsden J, Wheeler-Kingshott C, Sahyoun C, Matthews P, et al. Identifying brain regions for integrative sensorimotor processing with ankle movements.

Experimental brain research. 2005;166(1):31-42.

39. Dobkin BH, Firestine A, West M, Saremi K, Woods R. Ankle dorsiflexion as an fMRI paradigm to assay motor control for walking during rehabilitation. Neuroimage. 2004;23(1):370-81.

40. Maclntosh BJ, Mraz R, Baker N, Tam F, Staines WR, Graham SJ. Optimizing the experimental design for ankle dorsiflexion fMRI. Neuroimage. 2004;22(4):1619-27.

41. Orlovsky $\mathrm{G}$. The effect of different descending systems on flexor and extensor activity during locomotion. Brain research. 1972;40(2):359-72. 
42. Armstrong $D$, Drew $T$. Forelimb electromyographic responses to motor cortex stimulation during locomotion in the cat. The Journal of physiology. 1985;367(1):327-51.

43. Rho M-J, Lavoie S, Drew T. Effects of red nucleus microstimulation on the locomotor pattern and timing in the intact cat: a comparison with the motor cortex. Journal of neurophysiology. 1999;81(5):2297-315.

44. Grillner S. Control of locomotion in bipeds, tetrapods, and fish. Comprehensive Physiology. 2011.

45. Jayaram G, Stinear JW. The effects of transcranial stimulation on paretic lower limb motor excitability during walking. Journal of Clinical Neurophysiology. 2009;26(4):272-9.

46. Kim CR, Kim D-Y, Kim LS, Chun MH, Kim SJ, Park CH. Modulation of cortical activity after anodal transcranial direct current stimulation of the lower limb motor cortex: a functional MRI study. Brain Stimulation: Basic, Translational, and Clinical Research in Neuromodulation. 2012;5(4):462-7.

47. Nonnekes J, Arrogi A, Munneke MA, van Asseldonk EH, Nijhuis LBO, Geurts AC, et al. Subcortical structures in humans can be facilitated by transcranial direct current stimulation. PloS one. 2014;9(9):e107731.

48. van Asseldonk EH, Boonstra TA. Transcranial direct current stimulation of the leg motor cortex enhances coordinated motor output during walking with a large inter-individual variability. Brain stimulation. 2016;9(2):182-90.

49. Kisner C, Colby LA, Borstad J. Therapeutic exercise: foundations and techniques: Fa Davis; 2017.

50. Lemon RN. Descending pathways in motor control. Annu Rev Neurosci. 2008;31:195-218.

51. Rea P. Essential clinical anatomy of the nervous system: Academic Press; 2015.

52. Daubney ME, Culham EG. Lower-extremity muscle force and balance performance in adults aged 65 years and older. Physical therapy. 1999;79(12):1177-85.

53. Gribble PA, Tucker WS, White PA. Time-of-day influences on static and dynamic postural control. Journal of athletic training. 2007;42(1):35.

54. Jacobs J, Horak F. Cortical control of postural responses. Journal of neural transmission. 2007;114(10):1339.

55. Hoogkamer W, Bruijn SM, Sunaert S, Swinnen SP, Van Calenbergh F, Duysens J. Adaptation and aftereffects of split-belt walking in cerebellar lesion patients. Journal of neurophysiology. 2015;114(3):1693-704.

56. Thach WT, Bastian AJ. Role of the cerebellum in the control and adaptation of gait in health and disease. Progress in brain research. 143: Elsevier; 2004. p. 353-66.

57. Sanes JN, Donoghue JP. Plasticity and primary motor cortex. Annual review of neuroscience. 2000;23(1):393-415.

58. Cohen LG, Celnik P, Pascual-Leone A, Corwell B, Faiz L, Dambrosia J, et al. Functional relevance of cross-modal plasticity in blind humans. Nature. 1997;389(6647):180.

59. Nudo RJ. Plasticity. NeuroRx. 2006;3(4):420-7.

60. Levin MF, Kleim JA, Wolf SL. What do motor "recovery" and "compensation" mean in patients following stroke? Neurorehabilitation and neural repair. 2009;23(4):313-9.

61. Heidelberger R, Shouval H, Zucker RS, Byrne JH. Synaptic Plasticity. From Molecules to Networks (Third Edition): Elsevier; 2014. p. 533-61.

62. Kessels HW, Malinow R. Synaptic AMPA receptor plasticity and behavior. Neuron. 2009;61(3):340-50.

63. Bernardinelli Y, Nikonenko I, Muller D. Structural plasticity: mechanisms and contribution to developmental psychiatric disorders. Frontiers in neuroanatomy. 2014;8:123.

64. Howland JG, Wang YT. Synaptic plasticity in learning and memory: stress effects in the hippocampus. Progress in brain research. 2008;169:145-58.

65. Bredt DS, Nicoll RA. AMPA receptor trafficking at excitatory synapses. Neuron. 2003;40(2):361-79. 
66. Newpher TM, Ehlers MD. Glutamate receptor dynamics in dendritic microdomains. Neuron. 2008;58(4):472-97.

67. Chung HJ, Xia J, Scannevin RH, Zhang X, Huganir RL. Phosphorylation of the AMPA receptor subunit GluR2 differentially regulates its interaction with PDZ domain-containing proteins. Journal of Neuroscience. 2000;20(19):7258-67.

68. Barry MF, Ziff EB. Receptor trafficking and the plasticity of excitatory synapses. Current opinion in neurobiology. 2002;12(3):279-86.

69. Rioult-Pedotti M-S, Friedman D, Donoghue JP. Learning-induced LTP in neocortex. Science. 2000;290(5491):533-6.

70. Cooke S, Bliss T. Plasticity in the human central nervous system. Brain. 2006;129(7):1659-73.

71. Gu Q. Neuromodulatory transmitter systems in the cortex and their role in cortical plasticity. Neuroscience. 2002;111(4):815-35.

72. Citri A, Malenka RC. Synaptic plasticity: multiple forms, functions, and mechanisms. Neuropsychopharmacology. 2008;33(1):18.

73. Malenka RC, Bear MF. LTP and LTD: an embarrassment of riches. Neuron. 2004;44(1):5-21.

74. Lisman JE. Three Ca2+ levels affect plasticity differently: the LTP zone, the LTD zone and no man's land. The Journal of physiology. 2001;532(2):285-.

75. Martin SJ, Grimwood PD, Morris RG. Synaptic plasticity and memory: an evaluation of the hypothesis. Annual review of neuroscience. 2000;23(1):649-711.

76. Buonomano DV, Merzenich MM. Cortical plasticity: from synapses to maps. Annual review of neuroscience. 1998;21(1):149-86.

77. Nudo R. Adaptive plasticity in motor cortex: implications for rehabilitation after brain injury. Journal of Rehabilitation Medicine-Supplements. 2003;41:7-10.

78. Horton JC, Fahle M, Mulder T, Trauzettel-Klosinski S. Adaptation, perceptual learning, and plasticity of brain functions. Graefe's Archive for Clinical and Experimental Ophthalmology. 2017;255(3):435-47.

79. Lloyd DM, Shore DI, Spence C, Calvert GA. Multisensory representation of limb position in human premotor cortex. Nature neuroscience. 2003;6(1):17.

80. Schaefer N, Rotermund C, Blumrich Em, Lourenco MV, Joshi P, Hegemann RU, et al. The malleable brain: plasticity of neural circuits and behavior-A review from students to students. Journal of neurochemistry. 2017.

81. Liepert J, Tegenthoff M, Malin J-P. Changes of cortical motor area size during immobilization. Electroencephalography and clinical neurophysiology/electromyography and motor control. 1995;97(6):382-6.

82. Lohse K, Wadden K, Boyd L, Hodges N. Motor skill acquisition across short and long time scales: a meta-analysis of neuroimaging data. Neuropsychologia. 2014;59:130-41.

83. Coynel D, Marrelec G, Perlbarg V, Pélégrini-Issac M, Van de Moortele P-F, Ugurbil K, et al. Dynamics of motor-related functional integration during motor sequence learning. Neuroimage. 2010;49(1):759-66.

84. Lehéricy $\mathrm{S}$, Benali $\mathrm{H}$, Van de Moortele P-F, Pélégrini-Issac $\mathrm{M}$, Waechter $\mathrm{T}$, Ugurbil $\mathrm{K}$, et al. Distinct basal ganglia territories are engaged in early and advanced motor sequence learning. Proceedings of the National Academy of Sciences of the United States of America. 2005;102(35):12566-71.

85. Sun FT, Miller LM, Rao AA, D'esposito M. Functional connectivity of cortical networks involved in bimanual motor sequence learning. Cerebral cortex. 2006;17(5):1227-34.

86. Greenough WT, Larson JR, Withers GS. Effects of unilateral and bilateral training in a reaching task on dendritic branching of neurons in the rat motor-sensory forelimb cortex. Behavioral and neural biology. 1985;44(2):301-14.

87. Kolb B, Cioe J, Comeau W. Contrasting effects of motor and visual spatial learning tasks on dendritic arborization and spine density in rats. Neurobiology of learning and memory.

2008;90(2):295-300. 
88. Costa RM, Cohen D, Nicolelis MA. Differential corticostriatal plasticity during fast and slow motor skill learning in mice. Current Biology. 2004;14(13):1124-34.

89. Rioult-Pedotti M-S, Friedman D, Hess G, Donoghue JP. Strengthening of horizontal cortical connections following skill learning. Nature neuroscience. 1998;1(3):230.

90. Monfils M-H, Teskey G. Skilled-learning-induced potentiation in rat sensorimotor cortex: a transient form of behavioural long-term potentiation. Neuroscience. 2004;125(2):329-36.

91. Ziemann U, lliać TV, Pauli C, Meintzschel F, Ruge D. Learning modifies subsequent induction of long-term potentiation-like and long-term depression-like plasticity in human motor cortex. Journal of Neuroscience. 2004;24(7):1666-72.

92. Stefan K, Wycislo M, Gentner R, Schramm A, Naumann M, Reiners K, et al. Temporary occlusion of associative motor cortical plasticity by prior dynamic motor training. Cerebral Cortex. 2005;16(3):376-85.

93. Rosenkranz K, Kacar A, Rothwell JC. Differential modulation of motor cortical plasticity and excitability in early and late phases of human motor learning. Journal of Neuroscience. 2007;27(44):12058-66.

94. Hall E, Flament D, Fraser C, Lemon R. Non-invasive brain stimulation reveals reorganised cortical outputs in amputees. Neuroscience letters. 1990;116(3):379-86.

95. Cohen LG, Bandinelli S, Findley TW, Hallett M. Motor reorganization after upper limb amputation in man: a study with focal magnetic stimulation. Brain. 1991;114(1):615-27.

96. Topka H, Cohen LG, Cole RA, Hallett M. Reorganization of corticospinal pathways following spinal cord injury. Neurology. 1991;41(8):1276-.

97. Kraus D, Gharabaghi A. Neuromuscular plasticity: disentangling stable and variable motor maps in the human sensorimotor cortex. Neural plasticity. 2016;2016.

98. Hallett M. Transcranial magnetic stimulation: a primer. Neuron. 2007;55(2):187-99.

99. Fox MD, Buckner RL, White MP, Greicius MD, Pascual-Leone A. Efficacy of transcranial magnetic stimulation targets for depression is related to intrinsic functional connectivity with the subgenual cingulate. Biological psychiatry. 2012;72(7):595-603.

100. Barker A, Freeston I, Jalinous R, Jarratt J. Non-invasive stimulation of motor pathways within the brain using time-varying magnetic fields. Clinical Neurophysiology. 1985;61(3):S245-S6.

101. Rossi S, Hallett M, Rossini PM, Pascual-Leone A, Group SoTC. Safety, ethical considerations, and application guidelines for the use of transcranial magnetic stimulation in clinical practice and research. Clinical neurophysiology. 2009;120(12):2008-39.

102. Di Lazzaro V, Restuccia D, Oliviero A, Profice P, Ferrara L, Insola A, et al. Magnetic transcranial stimulation at intensities below active motor threshold activates intracortical inhibitory circuits. Experimental Brain Research. 1998;119(2):265-8.

103. Rothwell J, Hallett M, Berardelli A, Eisen A, Rossini P, Paulus W. Magnetic stimulation: motor evoked potentials. Electroencephalogr Clin Neurophysiol Suppl. 1999;52:97-103.

104. Kobayashi M, Pascual-Leone A. Transcranial magnetic stimulation in neurology. The Lancet Neurology. 2003;2(3):145-56.

105. Conforto AB, Marie SK, Cohen LG, Scaff M. Estimulação magnética transcraniana. Arquivos de Neuro-Psiquiatria. 2003;61(1):146-52.

106. Klomjai W, Katz R, Lackmy-Vallée A. Basic principles of transcranial magnetic stimulation

(TMS) and repetitive TMS (rTMS). Annals of physical and rehabilitation medicine. 2015;58(4):208-13.

107. Zago S, Ferrucci R, Fregni F, Priori A. Bartholow, Sciamanna, Alberti: pioneers in the electrical stimulation of the exposed human cerebral cortex. The Neuroscientist. 2008;14(5):521-8.

108. Utz KS, Dimova V, Oppenländer K, Kerkhoff G. Electrified minds: transcranial direct current stimulation (tDCS) and galvanic vestibular stimulation (GVS) as methods of non-invasive brain stimulation in neuropsychology-a review of current data and future implications. Neuropsychologia. 2010;48(10):2789-810.

109. Parent A. Giovanni Aldini: from animal electricity to human brain stimulation. Canadian journal of neurological sciences. 2004;31(4):576-84. 
110. Bindman L, Lippold O, Redfearn J. The action of brief polarizing currents on the cerebral cortex of the rat (1) during current flow and (2) in the production of long-lasting after-effects. The Journal of physiology. 1964;172(3):369-82.

111. Purpura DP, McMurtry JG. Intracellular activities and evoked potential changes during polarization of motor cortex. J Neurophysiol. 1965;28(1):166-85.

112. Priori A, Berardelli A, Rona S, Accornero N, Manfredi M. Polarization of the human motor cortex through the scalp. Neuroreport. 1998;9(10):2257-60.

113. Nitsche M, Paulus W. Excitability changes induced in the human motor cortex by weak transcranial direct current stimulation. The Journal of physiology. 2000;527(3):633-9.

114. Brunoni AR, Nitsche MA, Bolognini N, Bikson M, Wagner T, Merabet L, et al. Clinical research with transcranial direct current stimulation (tDCS): challenges and future directions. Brain Stimulation: Basic, Translational, and Clinical Research in Neuromodulation. 2012;5(3):175-95.

115. Soekadar SR, Witkowski M, Cossio EG, Birbaumer N, Cohen LG. Learned EEG-based brain selfregulation of motor-related oscillations during application of transcranial electric brain stimulation: feasibility and limitations. Frontiers in behavioral neuroscience. 2014;8.

116. Marquez J, Conley A, Karayanidis F, Lagopoulos J, Parsons M. Anodal direct current stimulation in the healthy aged: Effects determined by the hemisphere stimulated. Restorative neurology and neuroscience. 2015;33(4):509-19.

117. Liebetanz D, Nitsche MA, Tergau F, Paulus W. Pharmacological approach to the mechanisms of transcranial DC-stimulation-induced after-effects of human motor cortex excitability. Brain. 2002;125(10):2238-47.

118. Stagg CJ, Nitsche MA. Physiological basis of transcranial direct current stimulation. The Neuroscientist. 2011;17(1):37-53.

119. Nitsche M, Fricke K, Henschke U, Schlitterlau A, Liebetanz D, Lang N, et al. Pharmacological modulation of cortical excitability shifts induced by transcranial direct current stimulation in humans. The Journal of physiology. 2003;553(1):293-301.

120. Stagg C, O'shea J, Kincses Z, Woolrich M, Matthews P, Johansen-Berg H. Modulation of movement-associated cortical activation by transcranial direct current stimulation. European Journal of Neuroscience. 2009;30(7):1412-23.

121. Nitsche MA, Seeber A, Frommann K, Klein CC, Rochford C, Nitsche MS, et al. Modulating parameters of excitability during and after transcranial direct current stimulation of the human motor cortex. The Journal of physiology. 2005;568(1):291-303.

122. Ardolino G, Bossi B, Barbieri S, Priori A. Non-synaptic mechanisms underlie the after-effects of cathodal transcutaneous direct current stimulation of the human brain. The Journal of physiology. 2005;568(2):653-63.

123. Gillick BT, Zirpel L. Neuroplasticity: an appreciation from synapse to system. Archives of physical medicine and rehabilitation. 2012;93(10):1846-55.

124. Nitsche MA, Paulus W. Sustained excitability elevations induced by transcranial DC motor cortex stimulation in humans. Neurology. 2001;57(10):1899-901.

125. Batsikadze G, Moliadze V, Paulus W, Kuo M-F, Nitsche MA. Partially non-linear stimulation intensity-dependent effects of direct current stimulation on motor cortex excitability in humans. The Journal of physiology. 2013;591(7):1987-2000.

126. Monte-Silva K, Kuo M-F, Hessenthaler S, Fresnoza S, Liebetanz D, Paulus W, et al. Induction of late LTP-like plasticity in the human motor cortex by repeated non-invasive brain stimulation. Brain Stimulation. 2013;6(3):424-32.

127. Reis J, Robertson EM, Krakauer JW, Rothwell J, Marshall L, Gerloff C, et al. Consensus: can transcranial direct current stimulation and transcranial magnetic stimulation enhance motor learning and memory formation? Brain Stimulation: Basic, Translational, and Clinical Research in Neuromodulation. 2008;1(4):363-9.

128. Pascual-Leone A, Valls-Solé J, Wassermann EM, Hallett M. Responses to rapid-rate transcranial magnetic stimulation of the human motor cortex. Brain. 1994;117(4):847-58. 
129. Scholz J, Klein MC, Behrens TE, Johansen-Berg H. Training induces changes in white-matter architecture. Nature neuroscience. 2009;12(11):1370.

130. Landi SM, Baguear F, Della-Maggiore V. One week of motor adaptation induces structural changes in primary motor cortex that predict long-term memory one year later. Journal of Neuroscience. 2011;31(33):11808-13.

131. Buch ER, Santarnecchi E, Antal A, Born J, Celnik PA, Classen J, et al. Effects of tDCS on motor learning and memory formation: a consensus and critical position paper. Clinical Neurophysiology. 2017;128(4):589-603.

132. Reis J, Fritsch B. Modulation of motor performance and motor learning by transcranial direct current stimulation. Current opinion in neurology. 2011;24(6):590-6.

133. Stagg C, Jayaram G, Pastor D, Kincses Z, Matthews P, Johansen-Berg H. Polarity and timingdependent effects of transcranial direct current stimulation in explicit motor learning. Neuropsychologia. 2011;49(5):800-4.

134. Reis J, Schambra HM, Cohen LG, Buch ER, Fritsch B, Zarahn E, et al. Noninvasive cortical stimulation enhances motor skill acquisition over multiple days through an effect on consolidation. Proceedings of the National Academy of Sciences. 2009;106(5):1590-5.

135. Alonzo A, Brassil J, Taylor JL, Martin D, Loo CK. Daily transcranial direct current stimulation (tDCS) leads to greater increases in cortical excitability than second daily transcranial direct current stimulation. Brain stimulation. 2012;5(3):208-13.

136. Saucedo Marquez CM, Zhang X, Swinnen SP, Meesen R, Wenderoth N. Task-specific effect of transcranial direct current stimulation on motor learning. Frontiers in human neuroscience.

2013;7:333.

137. Ferrucci R, Mameli F, Guidi I, Mrakic-Sposta S, Vergari M, Marceglia S, et al. Transcranial direct current stimulation improves recognition memory in Alzheimer disease. Neurology. 2008;71(7):493-8.

138. Galea JM, Celnik P. Brain polarization enhances the formation and retention of motor memories. Journal of neurophysiology. 2009;102(1):294-301.

139. Cogiamanian F, Vergari M, Pulecchi F, Marceglia S, Priori A. Effect of spinal transcutaneous direct current stimulation on somatosensory evoked potentials in humans. Clinical Neurophysiology. 2008;119(11):2636-40.

140. Winkler T, Hering P, Straube A. Spinal DC stimulation in humans modulates post-activation depression of the H-reflex depending on current polarity. Clinical neurophysiology. 2010;121(6):95761.

141. Fritsch B, Reis J, Martinowich K, Schambra HM, Ji Y, Cohen LG, et al. Direct current stimulation promotes BDNF-dependent synaptic plasticity: potential implications for motor learning. Neuron. 2010;66(2):198-204.

142. Antal A, Nitsche MA, Kincses TZ, Kruse W, Hoffmann KP, Paulus W. Facilitation of visuo-motor learning by transcranial direct current stimulation of the motor and extrastriate visual areas in humans. European Journal of Neuroscience. 2004;19(10):2888-92.

143. Thirugnanasambandam N, Grundey J, Paulus W, Nitsche MA. Dose-dependent nonlinear effect of L-DOPA on paired associative stimulation-induced neuroplasticity in humans. Journal of Neuroscience. 2011;31(14):5294-9.

144. López-Alonso V, Cheeran B, Río-Rodríguez D, Fernández-del-Olmo M. Inter-individual Variability in Response to Non-invasive Brain Stimulation Paradigms. Brain stimulation. 2014. 145. Wiethoff S, Hamada M, Rothwell JC. Variability in response to transcranial direct current stimulation of the motor cortex. Brain stimulation. 2014;7(3):468-75.

146. Woods AJ, Antal A, Bikson M, Boggio PS, Brunoni AR, Celnik P, et al. A technical guide to tDCS, and related non-invasive brain stimulation tools. Clinical Neurophysiology. 2016;127(2):1031-48.

147. Bikson M, Rahman A, Datta A. Computational models of transcranial direct current stimulation. Clinical EEG and Neuroscience. 2012;43(3):176-83. 
148. Ruffini G, Fox MD, Ripolles O, Miranda PC, Pascual-Leone A. Optimization of multifocal transcranial current stimulation for weighted cortical pattern targeting from realistic modeling of electric fields. Neurolmage. 2014;89:216-25.

149. Saturnino GB, Antunes A, Thielscher A. On the importance of electrode parameters for shaping electric field patterns generated by tDCS. Neuroimage. 2015;120:25-35.

150. Minhas P, Bansal V, Patel J, Ho JS, Diaz J, Datta A, et al. Electrodes for high-definition transcutaneous DC stimulation for applications in drug delivery and electrotherapy, including tDCS. Journal of neuroscience methods. 2010;190(2):188-97.

151. Kronberg G, Bikson M, editors. Electrode assembly design for transcranial direct current stimulation: a FEM modeling study. Engineering in Medicine and Biology Society (EMBC), 2012 Annual International Conference of the IEEE; 2012: IEEE.

152. Dmochowski JP, Datta A, Bikson M, Su Y, Parra LC. Optimized multi-electrode stimulation increases focality and intensity at target. Journal of neural engineering. 2011;8(4):046011.

153. Sadleir RJ, Vannorsdall TD, Schretlen DJ, Gordon B. Target optimization in transcranial direct current stimulation. Frontiers in psychiatry. 2012;3.

154. Wagner S, Rampersad S, Aydin Ü, Vorwerk J, Oostendorp T, Neuling T, et al. Investigation of tDCS volume conduction effects in a highly realistic head model. Journal of neural engineering. 2013;11(1):016002.

155. Fox PT, Narayana S, Tandon N, Sandoval H, Fox SP, Kochunov P, et al. Column-based model of electric field excitation of cerebral cortex. Human brain mapping. 2004;22(1):1-14.

156. Kammer T, Vorwerg M, Herrnberger B. Anisotropy in the visual cortex investigated by neuronavigated transcranial magnetic stimulation. Neuroimage. 2007;36(2):313-21.

157. Jamil A, Batsikadze G, Kuo HI, Labruna L, Hasan A, Paulus W, et al. Systematic evaluation of the impact of stimulation intensity on neuroplastic after-effects induced by transcranial direct current stimulation. The Journal of physiology. 2017;595(4):1273-88.

158. Nitsche MA, Liebetanz D, Lang N, Antal A, Tergau F, Paulus W. Safety criteria for transcranial direct current stimulation (tDCS) in humans. Clinical Neurophysiology. 2003;114(11):2220-2.

159. Kang N, Summers JJ, Cauraugh JH. Transcranial direct current stimulation facilitates motor learning post-stroke: a systematic review and meta-analysis. Journal of Neurology, Neurosurgery \& Psychiatry. 2016;87(4):345-55.

160. Ito M. The cerebellum: brain for an implicit self: FT press; 2012.

161. Grimaldi G, Argyropoulos G, Boehringer A, Celnik P, Edwards M, Ferrucci R, et al. Noninvasive cerebellar stimulation-a consensus paper. The Cerebellum. 2014;13(1):121-38.

162. Madhavan S, Stinear JW. Focal and bidirectional modulation of lower limb motor cortex using anodal transcranial direct current stimulation. Brain stimulation. 2010;3(1):42-50.

163. Roche N, Lackmy A, Achache V, Bussel B, Katz R. Effects of anodal transcranial direct current stimulation over the leg motor area on lumbar spinal network excitability in healthy subjects. The Journal of physiology. 2011;589(11):2813-26.

164. Shah B, Nguyen TT, Madhavan S. Polarity independent effects of cerebellar tDCS on short term ankle visuomotor learning. Brain Stimulation: Basic, Translational, and Clinical Research in Neuromodulation. 2013;6(6):966-8.

165. Devanathan D, Madhavan S. Effects of anodal tDCS of the lower limb M1 on ankle reaction time in young adults. Experimental brain research. 2016;234(2):377-85.

166. Sriraman A, Oishi T, Madhavan S. Timing-dependent priming effects of tDCS on ankle motor skill learning. Brain research. 2014;1581:23-9.

167. Tanaka S, Takeda K, Otaka Y, Kita K, Osu R, Honda M, et al. Single session of transcranial direct current stimulation transiently increases knee extensor force in patients with hemiparetic stroke. Neurorehabilitation and neural repair. 2011;25(6):565-9. 


\section{Acknowledgements}

First and foremost, I would like to thank God, without my faith and my beliefs it would have been impossible to develop this work so far from home and have kept the inspiration of doing science.

Thanks to Dr. Michael Nitsche. I am honored to have had him as my mentor. He has taught me so much over the course of this journey. Thanks for all the time, energy, unwavering support, generosity, and kindness. I will never forget his question when I moved to Germany "What have you left behind you at home? I want to know what it means to you“. During these years, he has been much more than a science advisor for me, he has showed me that you can be efficient (I wish one day I can be that fast in corrections of reports drafts), and still understand that behind a student there is always a life background. Thanks to the other members of my thesis committee, Prof. Dr. Andree Niklas and Prof. Dr. Siegmar Blumentritt, for their support and brilliant advice during my PhD. To Dr. Anirban Dutta, thank you for all the help with the neuroengineering and modelling parts of my dissertation.

Sincere thanks to the department of Clinical Neurophysiology, to Prof. Dr. Walter Paulus, for his guidance and support through these years. Special thanks to Ms. Marion Kurze for her kind help, smiling face, and warm hug every time I have returned to Göttingen. To Manuel Hewitt, I thank for his professional technical help. Thanks to my friends and colleagues at the department. Thanks for all of your smiles, supportive words, enthusiasm, and happy hours celebrations after work. I have learned a lot with them: Gabriel de Lara, Dr. Alex Whilier, Dr. Zsolt Turi, Dr. Ivan Alekseichunk and Annika Primaßin.

My thank you also goes to everyone at the Department of Psychology and Neurosciences, Leibniz Research Centre for Working Environment and Human 
Factors-Dortmund. Moving in to another city in the middle of the PhD, and re-starting a new life would not have been that easy without all the help of our previous secretary Marietta Widynski, the scientific advices of Dr. Min-Fang Kuo, and technical support of Andreas Volgmann and Mr. Flöring. A thank you to my colleagues: Ensiyeh Ghasemian-Shirvan, Dr. Asif Jamil, Dr. Fatemeh Yavari, Mohsen Mosayebi, Leila Farnad, Desmoond Abboada, Dr. Alireza Shahbabaie, and Harun Ar Rashid. We were the students' team that started over with Prof. Dr. Nitsche the Neuroscience department at IfADo, and being part of this group will be always a pleasure for me.

Warmest thanks to my Master thesis advisor Dr. Katia Monte-Silva for having built the bridge that allowed me to accomplish my dream of doing my PhD studies abroad, and to have introduced me to the non-invasive brain stimulation "world". To my friends Maíra Souza and Lorena Melo, you have shared with me the desire of being abroad, and all the fears and challenges involved on it.

Finally, and most importantly, I would like to thank my family and friends. I love you all. Thank you to my parents, Alberto (in memoriam) and Ana Paula, for teaching me the importance of education and hard work for something you want. Mom, it has been a difficult time with the ocean between us, but the meaning of all of this is to build a better life for us. To my sisters, Amanda (in memoriam) and Maria Paula, having lost Amanda during the course of my PhD studies showed me that unimaginable barriers can be transposed. Amanda, you and dad are the stars that guide my step every day, and the reason of my daily smile. To my dear aunts, Edna, Jô, Maria and Patricia and my beloved uncles, Henrique and Carlos. What I am today has a little of each one of them. To my cousins, Nathália, Mariana, Thaísa, Thiago and Sergio, that I have like brother, thank you for the best childhood memories and fellowship. 
A thank you to Christoph Busch, my partner, you are the best surprise that Germany gave me. You bring out of the best in me, protect me, and support every step of the way, thanks for showing me the Ruhrgebiet, and for giving me a family here. Thanks Irmgard, Jürgen, Miriam, Chris, David, Evelyne, Oma, Volker, Ben and Wagner's for having adopted me as a member of this amazing family.

Sincere thanks to the Brazilian community in Göttingen, I have met such amazing people there. Thanks Ellen Borges to be my angel and "aunt" since my first minute in Göttingen, without all your help I would never succeed. To Dr. Pedro Martins, you have been the best party partner ever; together we brought some of the Brazilian festivities to Germany and disseminated our culture here. To Monika and Lucas, thanks for bringing Maximilian to my life and to have supported me in one of the hardest moments I have had here.

Thanks to my old friends. Rafaela Sobral, Dyego Araújo, Valéria Santos and Gabriela Sena, for making my life a constant smile, for the adolescence full of joy and for staying with me all these years. Finha and Mago, I will never be able to describe how friendship can be that strong. Rafael Caldas, Larissa Carvalho, Adriane Cardim, Déborah Carthagenes and Déborah Wanderley, our paths came together at the beginning of my academic life and I am very grateful to have them by my side. Chuchu, you came to do your $\mathrm{PhD}$ near to me in a moment that I was almost giving up, thanks to have been my basis here. To all who once passed in my life and for thousand reasons did not remain, be sure that somehow you have made me a better person.

Lastly, thank you to all volunteers that took part on this research, without you it would not have come true. I wish the studies we have done together can really benefit the people who are suffering from neurological diseases, and make the world 
more wonderful in the future. Thank you to DAAD for grant funding that supported 3 years and a half of my PhD. 